Check for updates

Cite this: J. Mater. Chem. A, 2021, 9 , 19054

Received 2nd April 2021

Accepted 1st June 2021

DOI: $10.1039 / d 1 t a 02775 a$

rsc.li/materials-a

\section{The rise of flexible zinc-ion hybrid capacitors: advances, challenges, and outlooks}

\begin{abstract}
Cheng Wang, $\mathbb{D}+$ Xin Zeng, $(\mathbb{D} \dagger$ † Patrick J. Cullen and Zengxia Pei (D)*
The advent of flexible electronic devices has given rise to urgent demand for compatible flexible power sources. Zinc-ion hybrid capacitors (ZIHCs) combine the complementary advantages of zinc-ion batteries - for high energy density-and supercapacitors - for exceptional power density and cycling stability-and thus they have been vigorously studied as a very promising energy storage candidate in recent years. In this review, we systematically summarize the fundamental principles and recent progress of ZIHCs, including the critical challenges faced by electrode materials, electrolytes, and electrodeelectrolyte interfaces. Furthermore, special focus is given toward their functionalities (e.g., environmental adaptability, stimuli-responsive properties, and miniaturization) commensurate with the flexible devices. A perspective on future directions of flexible ZIHCS is also presented. This timely and comprehensive review is expected to give a fresh impetus toward the rational design and development of flexible ZIHCs to fully realize their potential as flexible energy storage devices.
\end{abstract}

\section{Introduction}

The burgeoning intelligent flexible electronic devices have gradually begun to revolutionize our society with their high-tech designs and unique multifunctional interactive experiences. In contrast to traditional rigid devices, flexible electronic devices can be twisted, bent, rolled, or stretched to a certain extent while maintaining their normal functions. ${ }^{1,2}$ To fulfill the full flexible prospects of these novel electronics, compatible flexible energy storage systems (ESSs) with high energy and power density, excellent mechanical strength, superior safety, and

School of Chemical and Biomolecular Engineering, The University of Sydney, Sydney, New South Wales, 2006, Australia. E-mail: zengxia.pei@sydney.edu.au

$\dagger$ These authors contributed equally to this work. long cycling life are urgently needed. ${ }^{3,4}$ Among all ESSs being scrutinized, supercapacitors (SCs) have received widespread attention in academia and industry in the last few decades because of their high power density $\left(>10 \mathrm{~kW} \mathrm{~kg}^{-1}\right)$, fast charging/discharging rate (within seconds), and long cycle life $\left(>10^{5}\right.$ cycles).$^{5}$ Based on their energy storage mechanisms, supercapacitors can be classified into two categories: electrical double-layer capacitors (EDLCs) and pseudo-capacitors. The charge storage in EDLCs is mainly based on the accumulation of pure static charge at the electrode-electrolyte interface, while pseudocapacitors rely on fast and reversible faradaic reactions occurring at and/or near the electrode surface (typically $<5 \mathrm{~nm}$ ). ${ }^{6}$ Utilization of only the surface layers of the electrodes makes the energy density of SCs far below the requirements of modern electronics. ${ }^{7}$

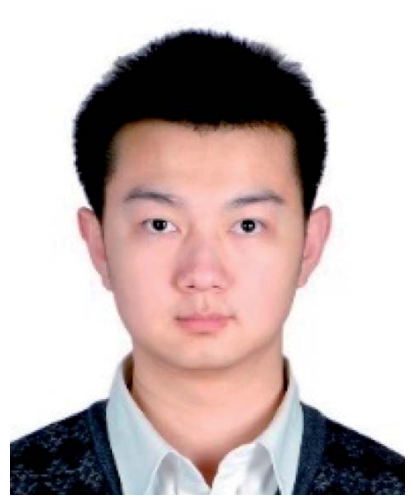

Cheng Wang received his Bachelor's degree in Applied Chemistry from Beijing University of Chemical Technology. He is currently pursuing his Ph.D. at the School of Chemical \& Biomolecular Engineering, the University of Sydney, Australia. His research interest is mainly on zinc-based energy storage devices.

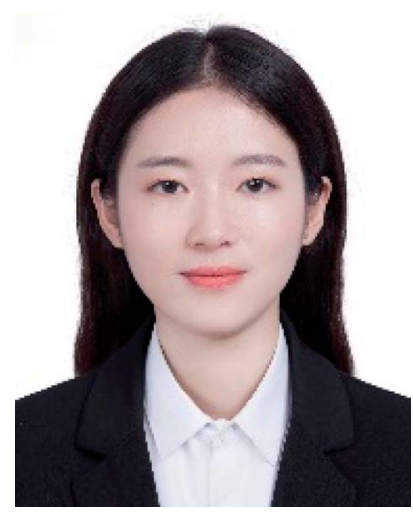

Xin Zeng received her $M$.Phil degree from the University of Sydney in 2019. She is currently pursuing her Ph.D. at the School of Chemical \& Biomolecular Engineering, the University of Sydney, Australia. Her research focuses on zinc-based flexible energy storage devices. 
To break the limitation of conventional SCs, hybrid capacitors with different asymmetric configurations have been developed to combine the complementary advantages of battery-type electrodes and capacitor-type electrodes. In this way, hybrid capacitors can provide high energy density while maintaining high power density and ultrastability, which also provides attractive solutions for the selection of energy devices. ${ }^{8}$ In other words, the proper design of hybrid capacitors can make up for specific problems associated with batteries or SCs. Historically, the concept of hybrid capacitors originates from the lithium-ion battery field, and lithium-ion hybrid capacitors were first reported by combining a lithium titanate $\left(\mathrm{Li}_{4} \mathrm{Ti}_{5} \mathrm{O}_{12}\right)$ negative electrode (NE) and an activated carbon positive electrode (PE). ${ }^{9,10}$ However, the limited reservation and uneven distribution of lithium $(\mathrm{Li})$ resources on the Earth greatly impede its practical applications in Li-based hybrid capacitors. Thus, recent years have witnessed the blossom of other hybrid capacitors based on monovalent and/or multivalent cations, including sodium $\left(\mathrm{Na}^{+}\right)$, potassium $\left(\mathrm{K}^{+}\right)$, magnesium $\left(\mathrm{Mg}^{2+}\right)$, zinc $\left(\mathrm{Zn}^{2+}\right)$, calcium $\left(\mathrm{Ca}^{2+}\right)$, and aluminum $\left(\mathrm{Al}^{3+}\right) \cdot{ }^{11-14}$ Fig. 1 summarizes the typical physical parameters of all these cations and metals. Among them, reactive metals such as $\mathrm{Na}$ and $\mathrm{K}$ increase crucial safety risks due to the requirement of organic electrolytes, whereas multivalent metal ions like $\mathrm{Mg}^{2+}, \mathrm{Ca}^{2+}$, and $\mathrm{Al}^{3+}$ suffer from sluggish diffusion and reaction kinetics. By contrast, since the invention of the first battery by Volta et al. in 1799, $\mathrm{Zn}$ metal has long been considered an ideal anode candidate merited by its easy processability, high electrical conductivity, high abundance, good compatibility/stability in water, non-flammability, low toxicity, and comparatively low price (ca. 2 USD per kg). ${ }^{\mathbf{1 5}, 16}$ More significantly, Zn metal possesses a high theoretical capacity $\left(820 \mathrm{~mA} \mathrm{~h} \mathrm{~g}^{-1}\right.$ and 5855 $\left.\mathrm{mA} \mathrm{h} \mathrm{cm}^{-3}\right)$, a suitable low redox potential $(-0.76 \mathrm{~V} v s$. standard hydrogen electrode (SHE)), and relatively low polarizability (compared with $\mathrm{Mg}$ and $\mathrm{Al}$ ). ${ }^{\mathbf{1 6 - 1 8}}$ All these advantages of zinc exemplify the tremendous potential of ZIHCs, which open a new horizon for the next-generation electrochemical ESSs.

In the last few years, encouraging strides have been achieved in ZIHCs. New mechanisms, emerging materials, and novel

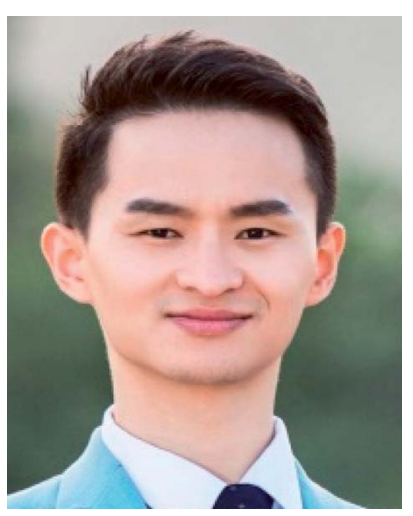

Zengxia Pei received his Ph.D. from City University of Hong Kong in 2017. He currently works as an Australian Research Council Discovery Early Career Researcher Award (ARC DECRA) Fellow at the University of Sydney. His research focuses on the design and development of nanomaterials for sustainable energy conversion and storage in fields including electrocatalysis, aqueous batteries/capacitors, and functional polyelectrolytes. He was recognized as one of the Australian Research Top 40 Rising Stars, and as a Clarivate Analytics Highly Cited Researcher in 2020. device designs are continuously being studied/developed, which are also reflected by the soaring number of publications in this domain. However, compared with other ESSs (e.g., conventional SCs and batteries), the development of ZIHCs is still in its infancy, and the dearth of understanding of the reaction process and interfacial properties requires further indepth investigations. In particular, though increasingly reported, the flexible devices with integrated functionalities bring in several new challenges. A timely and comprehensive review dedicated to flexible ZIHCs is, therefore, necessary to outline recent advances and rationalize future research directions. Herein, we first introduce the charge storage principles and relevant reaction mechanisms during the charging/discharging process. Then, the latest advances in the development of electrode materials, electrolytes, and device innovations are summarized and exemplified, with general design principles and special challenges/considerations for flexible ZIHCs highlighted. Finally, we provide our perspectives and constructively propose potential future research directions. We hope this review will provide inspirations and guidelines for further research studies on flexible ZIHCs.

\section{Principles of energy storage in supercapacitors and hybrid capacitors}

As mentioned above, the way supercapacitors store energy is mainly based on two types of capacitive behavior, one is EDLC that barely accumulates pure electrostatic charge at the electrode interface and the other is pseudocapacitance formed by prompt and reversible surficial redox reactions. There are already many comprehensive reviews summarizing the mechanisms of supercapacitors in detail..$^{4,7,20,21}$ In this section, we will succinctly summarize the characteristics of the electrochemical behaviors of SCs and batteries, then highlight the development history and performance metrics of ZIHCs.

\subsection{The energy storage mechanism of supercapacitors}

The energy density of conventional capacitors is very low because the charge storage area is very limited and the separation distance between the two charged plates also causes geometric constraints. The EDLC developed on this basis has a larger interfacial area, which expands the charge storage area. The separation distance of the charge is also within the atomic range so that the EDLC can store more energy. ${ }^{22}$ In the past, it was believed that to obtain a high capacitance, the pore size of the electrode material should be much larger than the size of the electrolyte ions and their solvated shells. However, later studies discovered that micropores and the nanostructure of the electrode material have a huge impact. ${ }^{23-25}$ Especially, Gogotsi and his colleagues ${ }^{24}$ discovered that the desolvated ions could contribute capacity in an EDLC when an electrode material (carbide-derived carbon) with pore sizes smaller than $1 \mathrm{~nm}$ was used. Following this, more experimental results prove that these solvated ions will be desolvated during the charging process, which makes the size of those ions smaller. These smaller ions can then utilize smaller holes in the electrode 


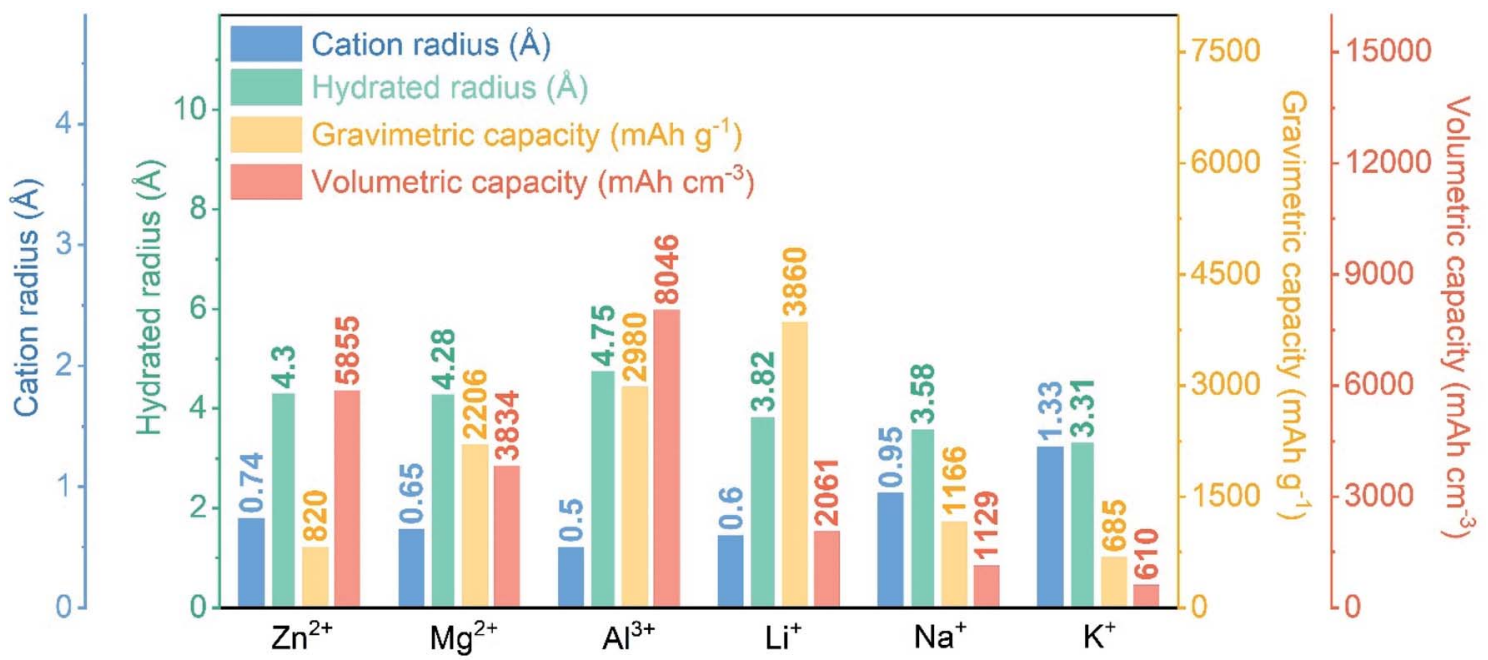

Fig. 1 A comparison of the cation radius, hydrated ion radius of typical charge carrier ions, and gravimetric and volumetric capacities of the corresponding metals. ${ }^{18,19}$

materials and improve the energy storage performance of EDLCs. $^{26,27}$ At the same time, many studies have found that when the ion size is comparable to the electrode material's pore size, the capacitance will also be greatly increased. ${ }^{28,29}$ Thus, the solvation and desolvation of ions and the interaction of ions under extreme confinement in pores have been the focus of research in recent years. ${ }^{24,30,31}$ The nano- and sub-nano-scale charge-storage mechanism have become a key research direction to improve the energy density of supercapacitors.

Typically, the energy storage of EDLCs purely relies on a high surface area electrode, which shows a potential-independent capacitance with a potential-independent current (Fig. 2a and b). The corresponding discharge curve of EDLCs is almost linear (Fig. 2c). In contrast, the battery materials typically exhibit prominent and distinctly separated peaks corresponding to the redox reactions in associated metal centers (Fig. $2 \mathrm{~g}$ and $\mathrm{h}$ ). Battery materials have a significant discharge plateau in the

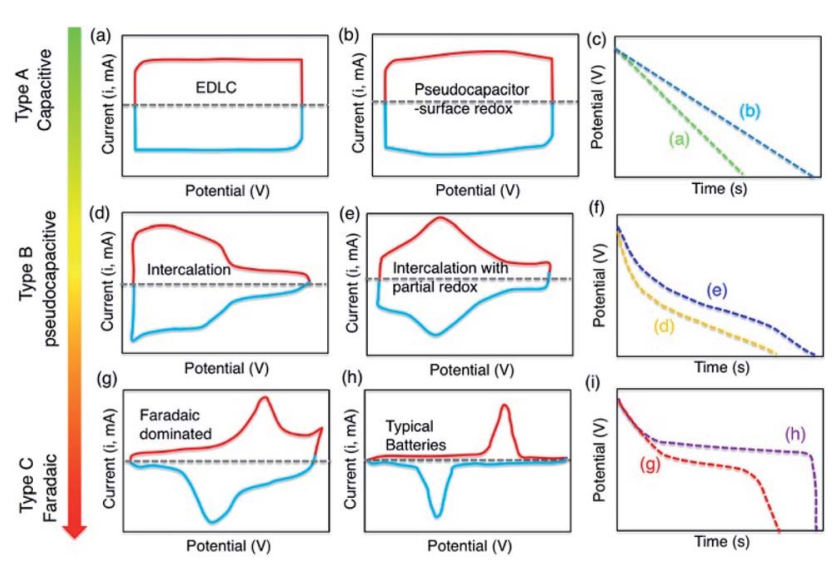

Fig. 2 Distinction between ( $a$ and b) EDLC; ( $d$ and e) pseudocapacitor and $(\mathrm{g}$ and $\mathrm{h}$ ) battery materials, with the corresponding discharge curves (c, $f$ and i). Reproduced with permission. ${ }^{32}$ Copyright 2018 , American Chemical Society. discharge curve (Fig. 2i), corresponding to the potential of faradaic reduction or oxidation of metal centers. ${ }^{32}$ Pseudocapacitors occupy a middle ground between EDLCs and batteries. Although the faradaic reactions occur in pseudocapacitors, their characteristics are expressed between the two extremes. Therefore, it is very important to understand the pseudocapacitance materials and the characteristics of pseudocapacitance.

In terms of pseudocapacitor electrode materials, their energy storage can proceed via faradaic electron transfer and nonfaradaic surficial charge adsorption. $\mathrm{RuO}_{2}$ and $\mathrm{MnO}_{2}$ are wellstudied representatives of pseudocapacitor materials. Materials such as doped carbon and conductive polymers are also potential candidates for pseudocapacitive electrodes. ${ }^{25,33,34}$ Regarding electrochemical behavior, the characteristics of either one (Fig. 2b, d and e) or a combination of each usually occur in pseudocapacitors that have neither a significant discharge plateau nor a linear relationship in the discharge curve. $^{32}$ These studies on the mechanism and behavior of EDLCs, pseudocapacitors, and batteries provide a solid foundation for a better understanding and design of hybrid capacitors that combine the supercapacitor- and battery-type electrodes.

\subsection{The energy storage mechanism of zinc ion hybrid capacitors}

The first demonstration of a ZIHC was done by Nohara et al. in $2007 .{ }^{35}$ However, they used a corrosive $\mathrm{KOH} / \mathrm{ZnO}$ solution as the electrolyte, so that the electrochemical performance of the ZIHC was pretty low in the initial stage with only 300 cycles and $70 \%$ capacitance retention. It is worth mentioning that they constructed the ZIHC using a capacitor-type PE (activated carbon) and a battery-like Zn metal NE. This structure has become a typical configuration of subsequent ZIHCs, as shown in Fig. 3a. Note that in numerous studies in the field of energy storage, the term NE refers to the anode and the PE refers to the cathode. However, for rechargeable energy storage devices, the 

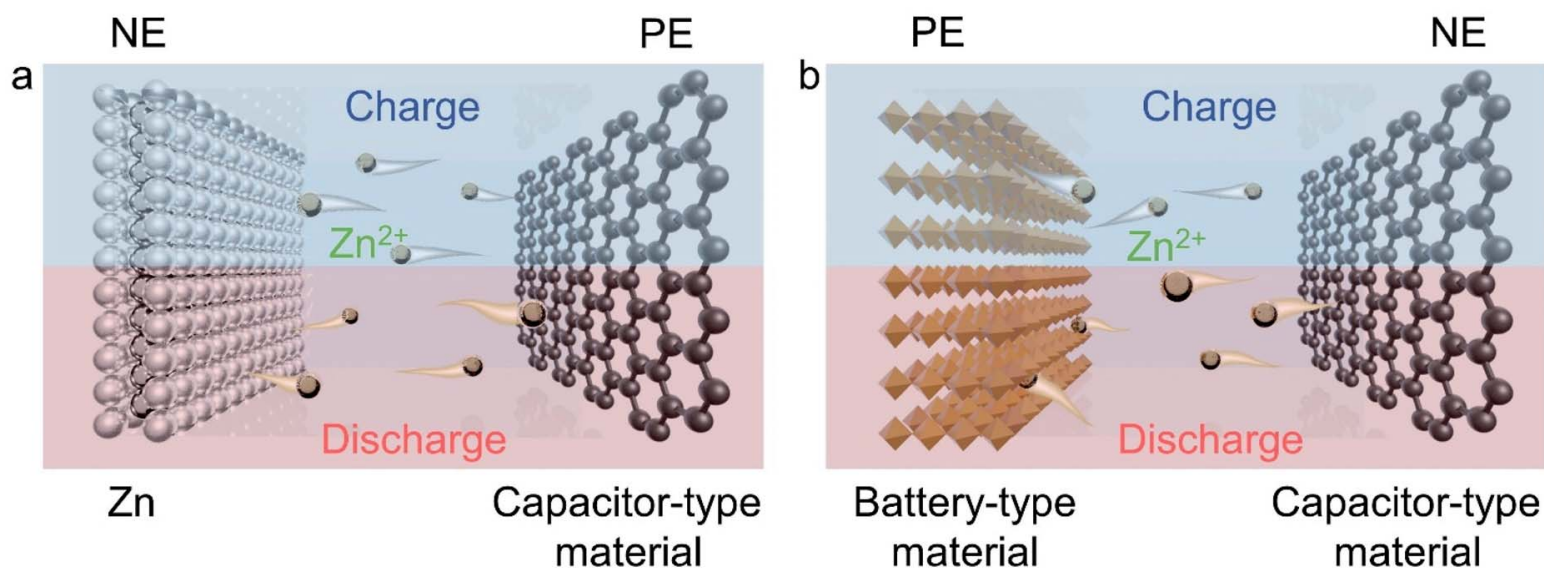

Fig. 3 Schematic diagrams of (a) the typical configuration of ZIHCs and (b) the "rocking-chair" configuration of ZIHCs, along with their corresponding electrochemical charge storage processes.

cathode and the anode switch with the charging/discharging process. $^{36}$ Therefore, to avoid possible misleading, we use the representation of $\mathrm{PE}$ and NE uniformly in this review.

As mentioned above, currently, most ZIHCs are composed of a capacitor-type electrode and a metallic Zn electrode with mild $\mathrm{Zn}$ salt solutions. The electrochemical reaction of $\mathrm{Zn} \mathrm{NE}$ in ZIHCs can be described as follows:

$$
\mathrm{Zn} \leftrightarrow \mathrm{Zn}^{2+}+2 \mathrm{e}^{-}
$$

For PEs, carbon materials are most widely used. It has been shown that in both the common activated carbon (AC) and Ndoped porous carbon electrodes, the capacitance is composed of a typical double-layer capacitance and a pseudocapacitance by chemical adsorption of $\mathrm{Zn}^{2+}$ ions. ${ }^{37-39}$ The behaviors of pseudocapacitance on the oxygen-containing groups in PEs can be summarized as follows:

$$
\begin{gathered}
\mathrm{C}-\mathrm{OH}+\mathrm{Zn}^{2+}+\mathrm{e}^{-} \leftrightarrow \mathrm{C} \cdots \mathrm{O} \cdots \mathrm{Zn}+\mathrm{H}^{+} \\
\mathrm{COOH}+\mathrm{Zn}^{2+}+\mathrm{e}^{-} \leftrightarrow \mathrm{COO}-\mathrm{Zn}+\mathrm{H}^{+}
\end{gathered}
$$

In addition to the main reactions on the electrodes, some parasitic reactions may occur. ${ }^{37,40}$ The typical reactions using the zinc sulfate $\left(\mathrm{ZnSO}_{4}\right)$ electrolyte can be described as follows:

$$
\begin{gathered}
6 \mathrm{H}_{2} \mathrm{O} \leftrightarrow 6 \mathrm{OH}^{-}+6 \mathrm{H}^{+} \\
4 \mathrm{Zn}^{2+}+6 \mathrm{OH}^{-}+\mathrm{SO}_{4}{ }^{2-}+0.5 \mathrm{H}_{2} \mathrm{O} \leftrightarrow \mathrm{Zn}_{4} \mathrm{SO}_{4}(\mathrm{OH})_{6} \cdot 0.5 \mathrm{H}_{2} \mathrm{O}(5) \\
\mathrm{Zn}_{4} \mathrm{SO}_{4}(\mathrm{OH})_{6} \cdot 0.5 \mathrm{H}_{2} \mathrm{O}+6 \mathrm{H}^{+}=4 \mathrm{Zn}^{2+}+\mathrm{SO}_{4}{ }^{2-}+6.5 \mathrm{H}_{2} \mathrm{O}
\end{gathered}
$$

Of some recent progress, $\mathrm{Hu}$ 's group ${ }^{\mathbf{4 1}}$ corroborated that in addition to heteroatoms in carbon materials, defects (e.g., carbon vacancy) can also be the adsorption sites for $\mathrm{Zn}^{2+}$ ions. Specifically, DFT calculations showed that the faradaic chargetransfer related chemisorption process is thermodynamically favorable in the defective vacancy sites of graphene compared with that in defect-free graphene. Also, the defect sites have high adsorption energy for both cations and anions (i.e., $\mathrm{Zn}^{2+}$ and $\mathrm{CF}_{3} \mathrm{SO}_{3}{ }^{-}$, respectively). Still, such a mechanism needs more in-depth investigations.

Another type (sometimes termed as the "rocking-chair" type) of ZIHC is constructed from a battery-type PE, a capacitor-type $\mathrm{NE}$, and a mild $\mathrm{Zn}$ salt solution (Fig. $3 \mathrm{~b}$ ). In this configuration, the reversible insertion/extraction of $\mathrm{Zn}^{2+}$ in $\mathrm{PE}$ and the adsorption and desorption of anions on $\mathrm{NE}$ are the main charge-storage mechanisms. ${ }^{42}$ Some researchers argue that this new structure avoids using excessive $\mathrm{Zn}$, which results in an overall increase in the device's specific capacity. ${ }^{\mathbf{4 3}}$ However, such assertions may only be reasonable on the assumption that the loading mass of active materials is comparable in ZIHCs.

\subsection{The performance metrics for zinc ion hybrid capacitors}

Some of the performance characteristics of ZIHCs are the same as those of supercapacitors: (1) high power density; (2) excellent cycling performance; (3) fast charging/discharging in seconds; (4) safe operation; and (5) low cost. However, there are significant differences in energy storage mechanisms between ZIHCs and conventional SCs, so that the evaluation criteria for the performance of ZIHCs are distinct from those for supercapacitors.

For ZIHCs, when the charging and discharging curves are linear, the specific capacity $\left(C_{\mathrm{m}}, \mathrm{mA} \mathrm{h} \mathrm{g}^{-1}\right)$ is calculated based on the following equation: $:^{38,42}$

$$
C_{\mathrm{m}}=\frac{I \times \Delta t}{3.6 m}
$$

wherein $I$ is the current (A), $\Delta t$ is the discharging time (s), and $m$ is the mass of the active material $(\mathrm{g})$.

The Coulombic efficiency $(\eta)$ is calculated as follows:

$$
\eta=\frac{t_{\mathrm{d}}}{t_{\mathrm{c}}}
$$

wherein $t_{\mathrm{d}}$ is the discharging time and $t_{\mathrm{c}}$ is the charging time (s).

The energy density $\left(E, \mathrm{~W} \mathrm{~h} \mathrm{~kg}{ }^{-1}\right)$ and the power density $(P, \mathrm{~W}$ $\mathrm{kg}^{-1}$ ) are calculated based on the following equations: 


$$
\begin{array}{r}
E=0.5 \times C_{\mathrm{m}} \Delta V \\
P=\frac{3600 \times E}{\Delta t}
\end{array}
$$

wherein $\Delta V$ is the potential window $(\mathrm{V})$.

When the charging and discharging curves are non-linear, the specific capacity $\left(C_{\mathrm{m}}, \mathrm{mA} \mathrm{h} \mathrm{g}{ }^{-1}\right)$ should be calculated by integrating the area under the discharging curve with the following equation:

$$
C_{\mathrm{m}}=\frac{2 I \int V \mathrm{~d} t}{3.6 m \Delta V}
$$

It is important to note that the performance of many ZIHCs is reported in the metric of capacitance. However, for ZIHCs containing battery electrodes, capacity $\left(\mathrm{mA} \mathrm{h} \mathrm{g}^{-1}\right)$ is the most appropriate expression rather than capacitance $\left(\mathrm{F} \mathrm{g}^{-1}\right)$, particularly when reporting the performance of a full cell containing a two-electrode device. ${ }^{44}$ Especially, when expressing a single electrode's performance, it is valuable to provide values for capacity even for commonly used EDLC materials. ${ }^{45}$ For pseudocapacitive materials, expressing the performance using capacity avoids obtaining a performance higher than the theoretical value. ${ }^{46}$ Also, when the potential window or electrode material changes, the capacitance values will not be comparable with each other, while $\mathrm{mA} \mathrm{h} \mathrm{g}^{-1}$ can be a consistent metric to compare the performance of different materials. ${ }^{47}$

\section{PEs for flexible zinc ion hybrid capacitors}

In ZIHCs, the PE is an important component. The development of novel electrode materials that can accommodate more zinc ions effectively is the most direct and efficacious way to improve the overall electrochemical performance of ZIHCs. Several traditional PE materials for supercapacitors have been studied as potential candidates for ZIHCs. However, the realization of flexible ZIHCs requires the electrodes to be flexible while achieving good electrochemical performance; therefore, more in-depth consideration is needed in the selection and structural design of electrode materials. Currently, most of the electrodes in flexible ZIHCs mainly consist of a flexible current collector combined with active materials. Some typical metal-type current collectors (e.g., copper, silver) commonly used in other flexible supercapacitors may become problematic in flexible ZIHCs because these metals will corrode the $\mathrm{Zn}$ metal (by forming copper-zinc or silver-zinc primary cells). In this context, carbon-based electrodes (e.g., carbon fibers or carbon nanotube (CNT) paper) stand out since they concurrently offer satisfying conductivity, mechanical flexibility, and chemical stability. Despite these, the ZIHC has become an emerging research domain for only a few years, and the eventual assembly considerations from a device level are in their infancy. As such, although flexible collectors play an important role in flexible ZIHCs, related research is still limited compared to those on electrode materials. Therefore, in the following section, we mainly highlight the design principles of active electrode materials in flexible ZIHCs and exemplify them with some most recent advances. Typical materials, including carbon materials, metal oxides, conducting polymers, along with some emerging materials, will be discussed; all the performance metrics will be benchmarked in Table 1.

\subsection{Carbon materials}

Carbon materials, such as CNTs, graphene, and porous carbon (PC), hold a premier position as the most common electrode materials in supercapacitors, merited by their unique advantages including excellent conductivity, large specific surface area (SSA), tunable porosity, lightweight, and high stability. ${ }^{\mathbf{1 , 2 0 , 4 8}}$ Generally, the charge storage capability of carbon materials is highly dependent on their SSA, surficial functional groups, porosity, and dopant/defect configurations.

3.1.1 CNTs. CNTs are one of the earliest candidates to be proven as a potential PE material in ZIHCs. For example, by introducing a large number of oxygen-containing functional groups onto the CNT surface, Wang and co-workers reported the first ZIHC with acceptable cycling performance (5000 cycles) in 2016. ${ }^{49}$ They found the oxygen functionalities on CNTs contributed to pseudocapacitance via reversibly reacting with $\mathrm{Zn}^{2+}$. Later, Sun et al. ${ }^{50}$ pioneered constructing a flexible zincion micro-supercapacitor (ZmSC) using commercially available CNT paper as both PE and current collectors. In their design, the CNT paper was taped onto a polyimide tape and cut into six intersecting microelectrodes, of which half serves as a positive material and the other half as current collectors for $\mathrm{Zn}$ stripping and plating (Fig. 4a). Upon external deformations, the assembled ZmSC can form a bending or twisting structure to some extent. More significantly, such flexible ZmSCs have no significant change in electrochemical properties even under a maximum bending of $180^{\circ}$ (Fig. $4 \mathrm{~b}$ and c). However, one key limitation for CNTs is their relatively small SSA, which leads to a moderate capacity of the assembled ZIHCs.

3.1.2 Graphene. Graphene has also been validated as an ideal candidate for electrode materials in flexible ZIHCs. Similar to CNTs, graphene can act as both electrodes and collectors. Due to the unique 2D structure, restacking of graphene is the biggest challenge that restricts their use in ZIHCs. To make full use of the interlayer space of graphene nanosheets, Zhu et al. ${ }^{51}$ reported a controlled graphene oxide (GO) film swelling strategy to avoid the irreversible $\pi-\pi$ stacking in graphene lamellae. The thus prepared free-standing graphene hydrogel films show good flexibility, which can be directly used as PEs in flexible ZIHCs. A capacity of $99.3 \mathrm{~mA} \mathrm{~h} \mathrm{~g} \mathrm{~g}^{-1}$ and a specific energy of $76.2 \mathrm{~W} \mathrm{~h} \mathrm{~kg}^{-1}$ were achieved by the resulting flexible ZIHCs. Zhang and co-workers ${ }^{52}$ constructed a freestanding composite carbon fiber consisting of reduced GO (rGO) and single-walled CNTs. The single-walled CNTs were added to prevent the complete restacking of rGO nanosheets, which enable a large ion-accessible surface area. Such a freestanding structure also avoids the usage of additional current collectors. As expected, the resulting fiber ZIHC delivered a volumetric capacitance of $104.5 \mathrm{~F} \mathrm{~cm}^{-3}$ at a current density of $400 \mathrm{~mA} \mathrm{~cm}^{-3}$ and a high energy density of $48.5 \mathrm{~mW} \mathrm{~h} \mathrm{~cm}{ }^{-3}$ at 
Table 1 Summary of configurations and electrochemical performances of various recently reported ZIHCs

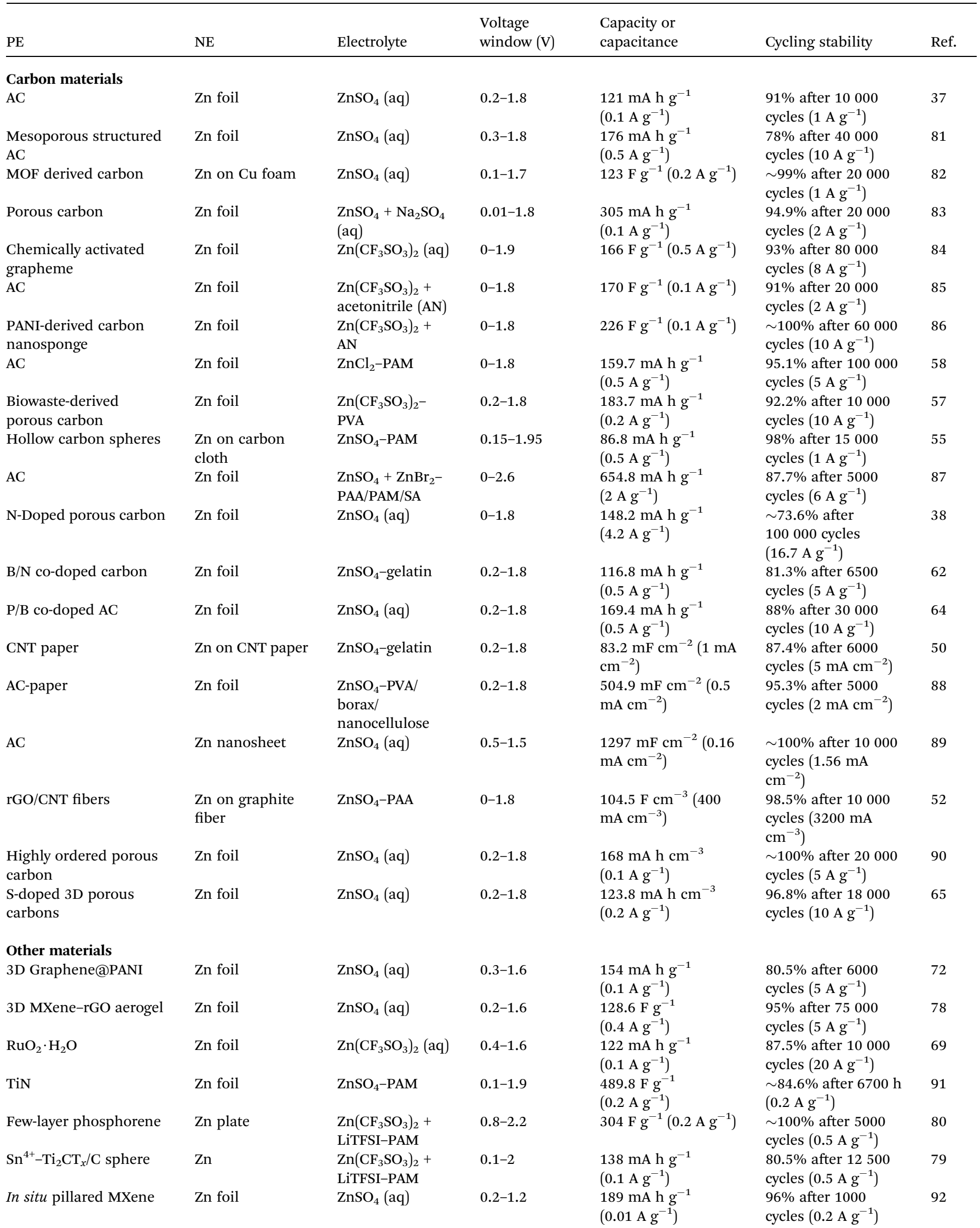


Table 1 (Contd.)

\begin{tabular}{|c|c|c|c|c|c|c|}
\hline $\mathrm{PE}$ & $\mathrm{NE}$ & Electrolyte & $\begin{array}{l}\text { Voltage } \\
\text { window (V) }\end{array}$ & $\begin{array}{l}\text { Capacity or } \\
\text { capacitance }\end{array}$ & Cycling stability & Ref. \\
\hline 3D-Printed MXene & $\begin{array}{l}\text { 3D-Printed CNT/ } \\
\mathrm{Zn}\end{array}$ & $\mathrm{ZnSO}_{4}(\mathrm{aq})$ & $0.1-1.2$ & $\begin{array}{l}1006.4 \mathrm{mF} \mathrm{cm}^{-2} \\
\left(0.38 \mathrm{~mA} \mathrm{~cm}^{-2}\right)\end{array}$ & $\begin{array}{l}86.5 \% \text { after } 6000 \\
\text { cycles }\left(10 \mathrm{~mA} \mathrm{~cm}^{-2}\right)\end{array}$ & 93 \\
\hline $\mathrm{MnO}_{2}$ nanorods & $\mathrm{AC}$ & $\mathrm{ZnSO}_{4}(\mathrm{aq})$ & $0-2$ & $\begin{array}{l}54.1 \mathrm{~mA} \mathrm{~h} \mathrm{~g}^{-1} \\
\left(0.1 \mathrm{~A} \mathrm{~g}^{-1}\right)^{a}\end{array}$ & $\begin{array}{l}65.3 \% \text { after } 3000 \\
\text { cycles }\end{array}$ & 43 \\
\hline $\mathrm{MnO}_{2}$ nanorods & $\mathrm{AC}$ & $\mathrm{Zn}\left(\mathrm{CF}_{3} \mathrm{SO}_{3}\right)_{2}(\mathrm{aq})$ & $0-2$ & $\begin{array}{l}46.4 \mathrm{~mA} \mathrm{~h} \mathrm{~g}^{-1} \\
\left(0.1 \mathrm{~A} \mathrm{~g}^{-1}\right)^{a}\end{array}$ & $\begin{array}{l}93.4 \% \text { after } 5000 \\
\text { cycles }\end{array}$ & 43 \\
\hline $\mathrm{V}_{2} \mathrm{O}_{5}$ & $\mathrm{AC}$ & $\mathrm{ZnSO}_{4}(\mathrm{aq})$ & $0-2$ & 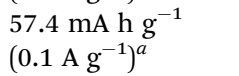 & $\begin{array}{l}97.3 \% \text { after } 6000 \\
\text { cycles }\left(0.5 \mathrm{~A} \mathrm{~g}^{-1}\right)\end{array}$ & 70 \\
\hline $\mathrm{MnO}_{2}-\mathrm{CNT}$ & MXene $\left(\mathrm{Ti}_{3} \mathrm{C}_{2} \mathrm{~T}_{x}\right)$ & $\begin{array}{l}\mathrm{ZnSO}_{4}+\mathrm{MnSO}_{4} \\
\text { (aq) }\end{array}$ & $0-1.9$ & $\begin{array}{l}115.1 \mathrm{~F} \mathrm{~g}^{-1} \\
\left.\mathrm{~s}^{-1}\right)^{a}\end{array}$ & $\begin{array}{l}\sim 83.6 \% \text { after } 15000 \\
\text { cycles }\left(5.224 \mathrm{~A} \mathrm{~g}^{-1}\right)\end{array}$ & 67 \\
\hline 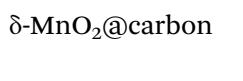 & MXene@cotton & $\begin{array}{l}\mathrm{ZnSO}_{4}+\mathrm{MnSO}_{4}- \\
\text { gelatin/borax }\end{array}$ & $0-1.9$ & $\begin{array}{l}172 \mathrm{~F} \mathrm{~g}^{-1}(1 \mathrm{mV} \\
\left.\mathrm{s}^{-1}\right)^{a}\end{array}$ & $\begin{array}{l}\sim 80.7 \% \text { after } 16000 \\
\text { cycles }\left(5.05 \mathrm{~A} \mathrm{~g}^{-1}\right)\end{array}$ & 94 \\
\hline EG/PANI & $\begin{array}{l}\text { POP-TAPP- } \\
\text { NTCA }\end{array}$ & $\mathrm{ZnSO}_{4}(\mathrm{aq})$ & $0.1-1.5$ & $\begin{array}{l}172 \mathrm{~F} \mathrm{~g}^{-1} \\
\left(0.13 \mathrm{~A} \mathrm{~g}^{-1}\right)^{a}\end{array}$ & $\begin{array}{l}90 \% \text { after } 1100 \\
\text { cycles }\left(0.3 \mathrm{~A} \mathrm{~g}^{-1}\right)\end{array}$ & 95 \\
\hline
\end{tabular}

a power density of $179.9 \mathrm{~mW} \mathrm{~cm}^{-3}$, which was the best performance among all ZIHCs by the time it was reported.

3.1.3 Porous carbon. In addition to the above two welldefined allotropes of carbon, PC is another most widely used electrode material in existing ZIHCs. In the last few decades, various protocols such as template-assisted methods (including hard and soft templates), activation methods, and molten salt methods, have been reported for the synthesis of PC. ${ }^{\mathbf{2 0 , 4 8 , 5 3 , 5 4}}$ These mature synthetic methods render PC desired and enriched component-structure features in a controlled manner, which depict great prospects for the usage of PC in zinc-ion storage. Dong et al. pioneered the use of a commercial AC (YP-50F) as electrode materials in ZIHCs. ${ }^{37}$ After coupling with $\mathrm{Zn}$ foil NE and $\mathrm{ZnSO}_{4}$ aqueous electrolyte, the assembled ZHIC delivered a capacity of $121 \mathrm{~mA} \mathrm{~h} \mathrm{~g}^{-1}$ at $0.1 \mathrm{~A} \mathrm{~g}^{-1}$ (corresponding to an energy density of $84 \mathrm{~W} \mathrm{~h} \mathrm{~kg} \mathrm{k}^{-1}$ ), a large power output of $14.9 \mathrm{~kW} \mathrm{~kg}^{-1}$, and decent cycling stability with $91 \%$ capacity

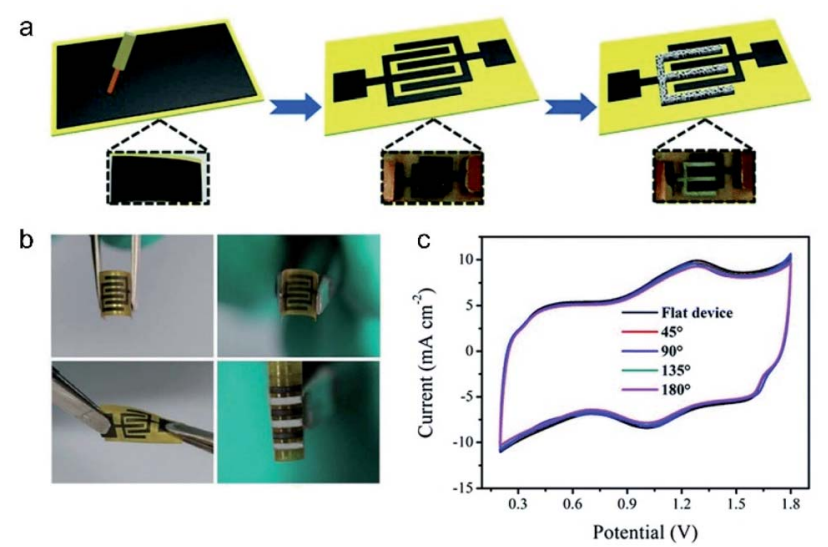

Fig. 4 (a) Schematic diagram of the construction of ZmSC. (b) Images of ZmSC in bending and twisting states. (c) Electrochemical properties at different degrees of bending. Reproduced with permission. ${ }^{50}$ Copyright 2018, The Royal Society of Chemistry. retention over 10000 cycles. Following this, Chen et al. ${ }^{55}$ constructed a spherical hollow PC by one-step carbonization of the hollow polyaniline-co-polypyrrole (PACP) spheres, which were synthesized using an emulsion method with a surfactant as the soft template (Fig. 5a-c). Due to the easily accessible adsorption sites, facilitated diffusion paths, and more electrolyte-electrode interfaces of the hollow PC, the constructed flexible ZIHCs achieved a maximum capacity of $86.8 \mathrm{~mA} \mathrm{~h} \mathrm{~g}{ }^{-1}$ at $0.5 \mathrm{~A} \mathrm{~g}^{-1}$. However, the SSA of this hollow PC $\left(819.5 \mathrm{~m}^{2} \mathrm{~g}^{-1}\right)$ is moderate compared with those of commercial AC (typically 1500-2500 $\mathrm{m}^{2}$ $\mathrm{g}^{-1}$ ), limiting the capacity of the carbon host. In view of this, Tang's group ${ }^{56}$ developed an in situ two-step activation strategy to boost the charge storage performance of PC. The assynthesized hierarchical porous activated carbon (HPAC) possesses an impressive SSA of up to $3525 \mathrm{~m}^{2} \mathrm{~g}^{-1}$, contributed by a mixture of micro- and mesopores. The resultant ZIHC based on the HPAC delivered a very high specific capacity of $231 \mathrm{~mA} \mathrm{~h} \mathrm{~g}^{-1}$ at $0.5 \mathrm{~A} \mathrm{~g}^{-1}$ and excellent rate capability with a retained capacity of $119 \mathrm{~mA} \mathrm{~h} \mathrm{~g}^{-1}$ at $20 \mathrm{~A} \mathrm{~g}^{-1}$, along with decent cycling stability with $70 \%$ capacity retention after 18000 cycles.

It should be noted that the increase of SSA will not necessarily lead to a larger capacity in proportion, since other parameters like porosity also have substantial impacts on the charge storage performance. In particular, it has been demonstrated that the EDLC shows an anomalous increase when the pore size gets closer to the ion size. ${ }^{24,28}$ Hence, considering the diameter of hydrated $\mathrm{Zn}^{2+}(0.86 \mathrm{~nm})$, the capacitance of PC materials could reach a peak value upon tuning the micropore size to around $0.9 \mathrm{~nm}$. To this end, Zhang's group ${ }^{57}$ reported a pencil shaving derived PC (PSC) with a narrow pore size distribution centered at 0.8, 1.1, and $1.2 \mathrm{~nm}$ (Fig. 5d and e). Though this PSC possesses a moderate SSA $\left(1293 \mathrm{~m}^{2} \mathrm{~g}^{-1}\right)$, a high storage capacitance/capacity of $413.3 \quad \mathrm{~F} \mathrm{~g}^{-1}$ / 183.7 $\mathrm{mA} \mathrm{h} \mathrm{g}^{-1}$ was achieved at $0.2 \mathrm{~A} \mathrm{~g}^{-1} \mathrm{using}$ it as positive materials in ZIHCs (PSC loading: $2 \mathrm{mg} \mathrm{cm}^{-2}$ ). Even at a high loading mass of $17 \mathrm{mg} \mathrm{\textrm {cm } ^ { - 2 }}$ of the PSC, the capacitance 


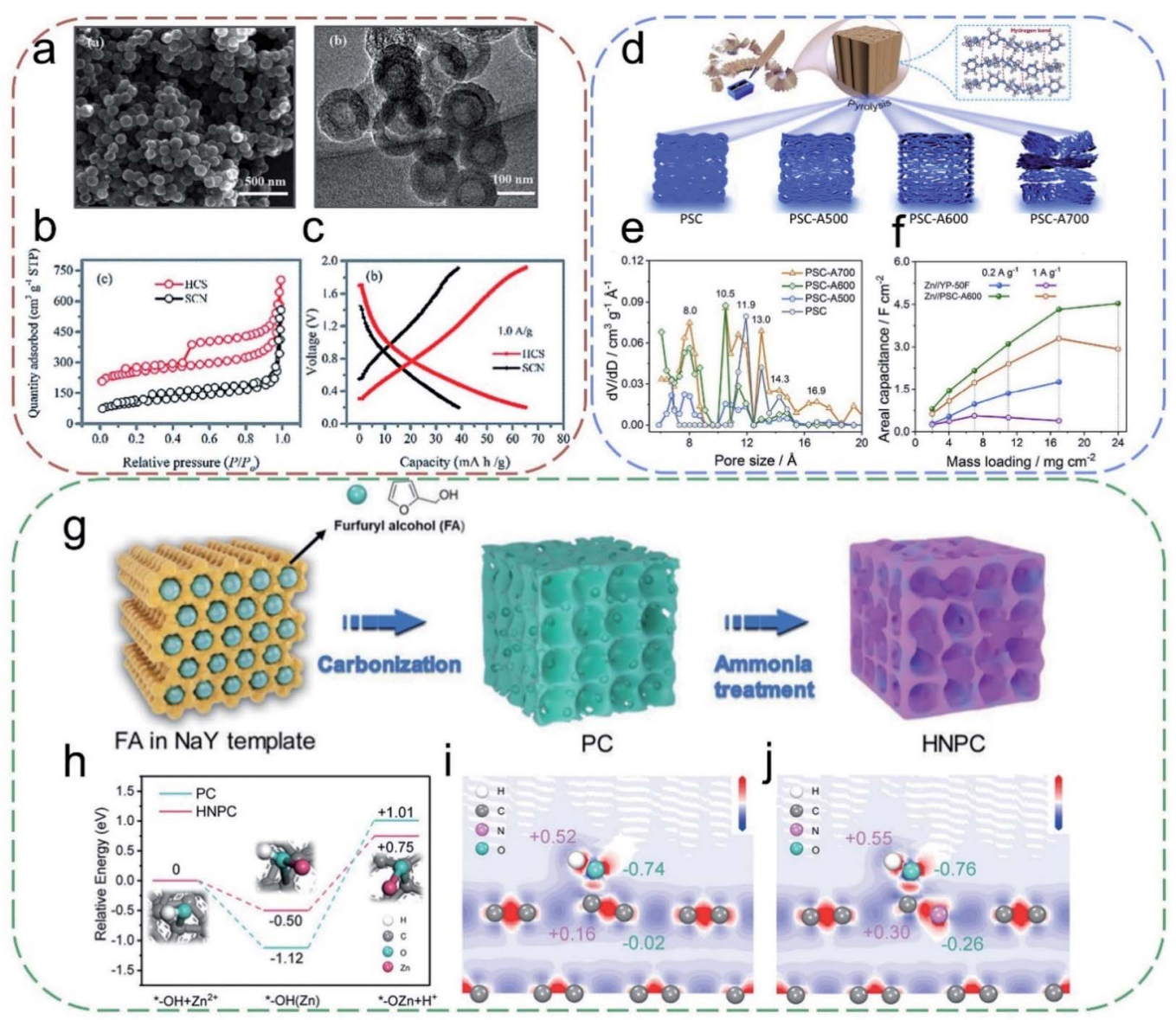

Fig. 5 (a) SEM and TEM images of hollow PC spheres. (b) $N_{2}$ adsorption and desorption isotherms. (c) Galvanostatic charge/discharge (GCD) curves at $1.0 \mathrm{~A} \mathrm{~g}^{-1}$. Reproduced with permission. ${ }^{55}$ Copyright 2019, The Royal Society of Chemistry. (d) Preparation of PSC samples. (e) Pore size distribution profiles. (f) Areal capacities at various mass loadings. Reproduced with permission. ${ }^{57}$ Copyright 2020, Elsevier. (g) Schematic of HNPC's synthesis route. (h) Relative energy distribution of the reaction pathway; insets show diagrams of the chemisorption pathways. (i) Electron density difference of PC. (j) Electron density difference of HNPC. Reproduced with permission. ${ }^{38}$ Copyright 2019, Wiley-VCH.

retention was as high as $61.6 \%$ (Fig. $5 \mathrm{f}$ ). Their results highlight that matching the porosity is an efficient way to boost the capacity of PC in ZIHCs. Similar conclusions were validated in another independent work by Wang and co-workers. ${ }^{58}$ On the other hand, despite enhancing the capacity, micropores will impede mass/ion transport as compared to mesopores and macropores. In this regard, constructing a hierarchical mixed porous structure was proposed as a potential solution toward addressing both the capacity and good rate capability. ${ }^{\mathbf{5 9 , 6 0}}$ For flexible devices, narrow pores are optimal where energy density is a premium consideration, whilst increasing the pore size is conducive when a pulse power supply is the main concern.

Apart from structural architecting, component engineering is the other main branch of commonly adopted strategies to enhance the electrochemical performances of PCs. Actually, these two strategies are not mutually exclusive but are always optimized simultaneously. For example, some typical activation processes (like activation by steam or $\mathrm{KOH}$ ) will inevitably introduce a substantial amount of surficial oxygen-containing groups in PCs. These polar groups are generally beneficial as they can enhance the surficial wettability of PCs and contribute to the pseudocapacitance. Other non-metallic heteroatom (e.g., $\mathrm{N}, \mathrm{B}, \mathrm{P}$, and $\mathrm{S}$ ) doping has also been considered to adjust the carbon materials' electronic and chemical properties for ZIHCs. ${ }^{61-66}$ Recently, Lu's group ${ }^{38}$ developed N-doped hierarchical PC (HNPC) materials as PEs for quasi-solid-state ZIHCs, using a two-step method involving template synthesis and subsequent $\mathrm{NH}_{3}$ activation (Fig. 5g). This HNPC possesses a high SSA (2762.7 $\mathrm{m}^{2} \mathrm{~g}^{-1}$ ) and delivered a specific capacity of $148.2 \mathrm{~mA} \mathrm{~h} \mathrm{~g}^{-1}$ even at a high current of $4.2 \mathrm{~A} \mathrm{~g}^{-1}$. The authors ascribed the exceptional performances to the N-doping-induced improved conductivity, surface wettability, and active sites. More significantly, they clarified the detailed chemical adsorption process of $\mathrm{Zn}^{2+}$ ions on HNPC (Fig. 5h). They found the rate-determining step of the whole $\mathrm{Zn}^{2+}$ ion adsorption process is the formation of the $\mathrm{C}-\mathrm{O}-\mathrm{Zn}$ bond. $\mathrm{N}$-doping can reduce this energy barrier from $2.13 \mathrm{eV}$ (in undoped PC) to $1.25 \mathrm{eV}$, thereby accelerating the adsorption process. Fig. $5 \mathrm{i}$ and $\mathrm{j}$ further confirm that the cleavage of the $\mathrm{O}-\mathrm{H}$ bond is more likely to happen after $\mathrm{N}$ doping, hence the $\mathrm{C}-\mathrm{O}-\mathrm{Zn}$ bond is more easily formed. Their results provide a rational guide for the heteroatom-doping strategy to improve the PCs' electrochemical performances in ZIHCs. In addition to the monoelement doping ( $\mathrm{N}$ or $\mathrm{S}$ ) strategy, dual- or even multi-element 
doping has also been studied to improve PCs' $\mathrm{Zn}^{2+}$ ion storage performance. ${ }^{62,64} \mathrm{~A}$ synergistic effect between the dopants is generally described to elucidate the improved performance of doped PC; however, the detailed mechanism is yet to be clearly uncovered.

In general, the unique advantages such as mature synthetic methods, large SSA, tunable porosity, and low prices endow PCs with great potential for large-scale use in ZIHCs. However, the low density and high porosity will also sacrifice the volumetric capacity/energy density and thus are detrimental to flexible ZIHC devices. The introduction of dopants can indeed boost the charge storage performance, but the doping sites (e.g., carbon atoms neighboring $\mathrm{O} / \mathrm{N}$ species) are also active sites for parasitic reactions (like the oxygen evolution reaction (OER)), which restricts the electrochemical window in the aqueous electrolyte particularly during the charging process. Therefore, overall considerations of these component-structure aspects are crucial to pursuing high-quality PCs for high power and energy density ZIHCs. Furthermore, most of the reported PCs exist as powder, so extra binders are mandatory for coating, which will incur a large internal resistance for the whole device. Developing freestanding carbon electrodes is a pertinent direction that deserves future endeavors.

\subsection{Transition metal oxides}

Transition metal oxide (TMO) materials are another common class of electrode materials for ZIHCs. The multiple valences of metal enable rich redox reactions for high theoretical specific capacity, making TMOs an ideal candidate to enhance the performance of flexible ZIHCs. Ma et al. ${ }^{43}$ demonstrated for the first time that $\mathrm{MnO}_{2}$ could be used as a PE material to construct ZIHCs (Fig. 6a). They demonstrated that the ZIHC coupling $\gamma$ $\mathrm{MnO}_{2}$ (PE) and AC (NE) could achieve an energy density comparable to that of the $\mathrm{Zn}-\mathrm{MnO}_{2}$ battery but with a remarkably larger power density (up to $13.0 \mathrm{~kW} \mathrm{~kg}^{-1}$ based on the total weight of $\gamma-\mathrm{MnO}_{2}$ nanorods and AC particles). The electrochemical performance of this new $\mathrm{MnO}_{2} / / 2 \mathrm{M} \mathrm{ZnSO}_{4} / / \mathrm{AC} \mathrm{ZIHC}$ was found to be dependent on the electrolyte. Upon the addition of extra $\mathrm{Mn}^{2+}$ ions to the $\mathrm{ZnSO}_{4}$ solution, the maximum capacity could be improved to $83.8 \mathrm{~mA} \mathrm{~h} \mathrm{~g}{ }^{-1}$; the anion replacement of $\mathrm{SO}_{4}{ }^{2-}$ by $\mathrm{CF}_{3} \mathrm{SO}_{3}{ }^{-}$suppresses manganese dissolution, which resulted in good cycling stability with $93.4 \%$ capacity retention after 5000 charging/discharging cycles. To fulfill the flexible functions, Wang et al. ${ }^{67}$ prepared $\mathrm{MnO}_{2}$-based flexible free-standing PEs ( $\mathrm{MnO}_{2}$-CNTs) by a facile vacuum membrane filtration method (Fig. 6c). The SEM image in Fig. 6d shows that the thickness of the synthesized $\mathrm{MnO}_{2}$-CNTs electrode is only $12.5 \mu \mathrm{m}$, enabling the constructed devices to be freely bent while maintaining the performance. The maximum energy and power densities of the flexible ZIHC exhibit high values of $98.6 \mathrm{~W} \mathrm{~h} \mathrm{~kg} \mathrm{~W}^{-1}\left(77.5 \mathrm{~W} \mathrm{~kg}^{-1}\right)$ and $2480.6 \mathrm{~W} \mathrm{~kg}-1$ (29.7 $\mathrm{W} \mathrm{h} \mathrm{kg}^{-1}$ ), respectively. Note $\mathrm{MnO}_{2}$ is known to suffer from severe structural changes during the charging/discharging cycles, which is one of the main reasons for its poor rate

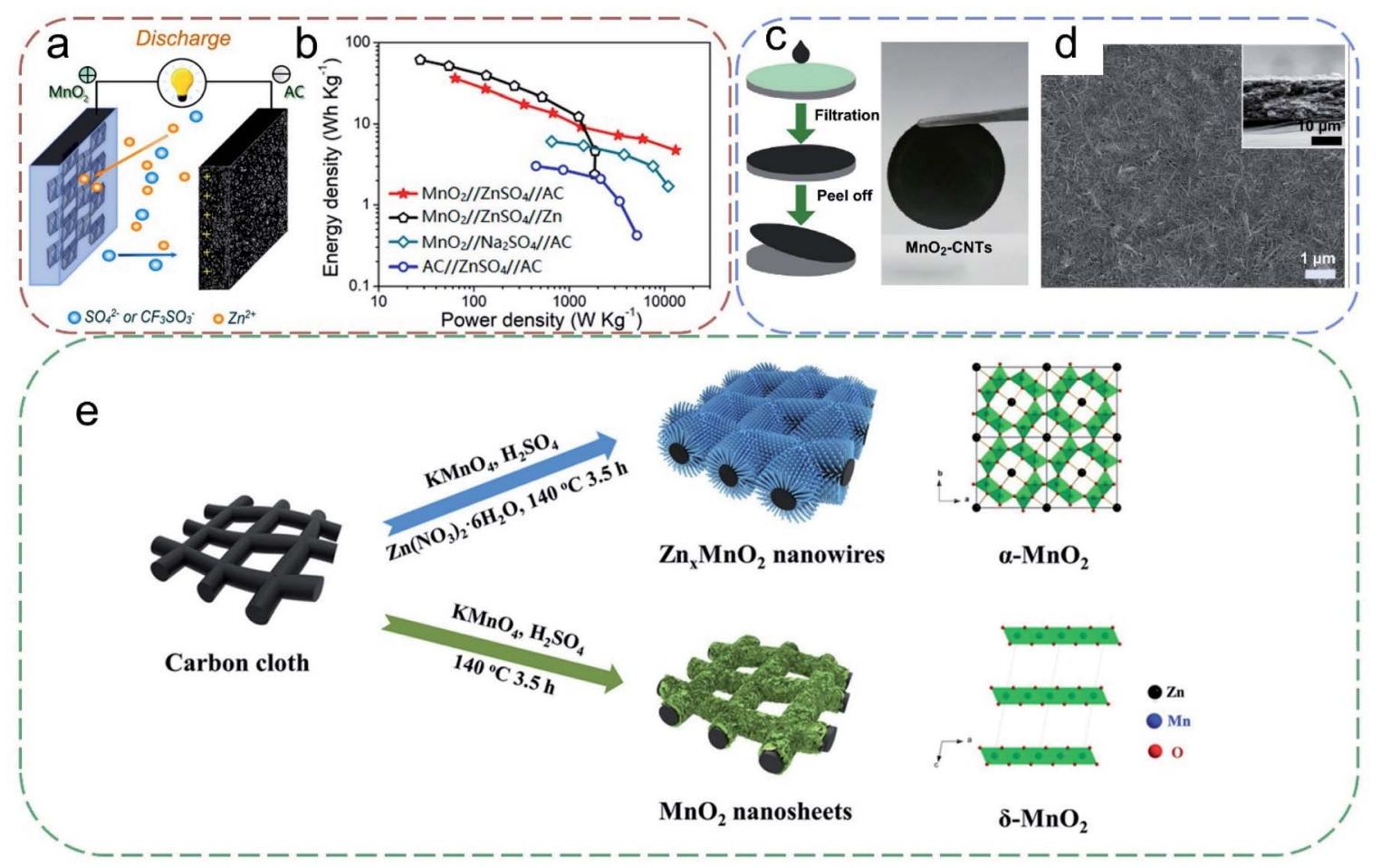

Fig. 6 (a) Schematic diagram of $\mathrm{MnO}_{2} / / \mathrm{AC} \mathrm{ZIHCs}$ and (b) the corresponding Ragone plots. Reproduced with permission. ${ }^{43}$ Copyright 2019 , Elsevier. (c) Fabrication procedure and a photograph of the free-standing $\left(\mathrm{MnO}_{2}-\mathrm{CNTS}\right) \mathrm{PE}$. (d) $\mathrm{SEM}$ image of $\mathrm{MnO}_{2}-\mathrm{CNTs} \mathrm{PE}$, with inset showing the thickness. Reproduced with permission. ${ }^{67}$ Copyright 2019, Springer Nature. (e) Schematic diagram of the fabrication of $\alpha-\mathrm{Zn}_{x} \mathrm{MnO}_{2}(\mathrm{acarbon}$ and $\delta-\mathrm{MnO}_{2}$ @acarbon cloth PEs. Reproduced with permission. ${ }^{68}$ Copyright 2020, Wiley-VCH. 
capability and durability. As such, Chen et al. ${ }^{68}$ reported that the pre-intercalation of $\mathrm{Zn}^{2+}$ could render a significant structural change in $\delta-\mathrm{MnO}_{2}$ to form a highly stable tunnel-structured $\mathrm{Zn}_{x} \mathrm{MnO}_{2}$ nanowires, which guarantee prompt and efficient intercalation/deintercalation of $\mathrm{Zn}^{2+}$ ions during the charging/ discharging process (Fig. 6e). The CC-supported $\mathrm{Zn}_{x} \mathrm{MnO}_{2}$ nanowire electrode was directly used with activated CC to assemble ZIHCs, which delivered an areal energy density of $969.9 \mu \mathrm{W} \mathrm{h} \mathrm{cm}{ }^{-2}$ and a maximum areal power density of 20.1 $\mathrm{mW} \mathrm{cm} \mathrm{cm}^{-2}$, along with a capacity retention of $83.1 \%$ over 5000 cycles. When constructed into a quasi-solid-state device, the flexible electrode endows a capacitance of $1446.6 \mathrm{mF} \mathrm{cm} \mathrm{cm}^{-2}$, a maximum areal energy density and a peak power density of $803.6 \mu \mathrm{W} \mathrm{h} \mathrm{cm}{ }^{-2}$ and $10.0 \mathrm{~mW} \mathrm{~cm}^{-2}$, respectively, making the ZIHCs reach up to the battery-level performances.

Similar to the case in $\mathrm{MnO}_{2}, \mathrm{Zn}^{2+}$ can also interact with other TMOs via pseudocapacitive reactions. For instance, amorphous $\mathrm{RuO}_{2} \cdot \mathrm{H}_{2} \mathrm{O}$ was used to construct ZIHCs by Dong and coauthors. ${ }^{69}$ By comparing with anhydrous $\mathrm{RuO}_{2}$ that is widely used as a supercapacitor material, they found that $\mathrm{RuO}_{2} \cdot \mathrm{H}_{2} \mathrm{O}$ exhibits higher capacity and better cycling stability in ZIHCs. The enhanced electrochemical performance was ascribed to the structured water $\left(\cdot \mathrm{H}_{2} \mathrm{O}\right)$, which promotes the pseudocapacitance reaction and maintained the electrode's high rate performance far beyond that of the $\mathrm{MnO}_{2}$ electrode. In another work, Ma et al. ${ }^{70}$ constructed ZIHCs using $\mathrm{V}_{2} \mathrm{O}_{5}$ as the PE materials, and the high stability of $\mathrm{V}_{2} \mathrm{O}_{5}$ enhanced the cycle life of ZIHCs compared to $\mathrm{MnO}_{2}$ counterparts.

Despite these inspiring signs of progress, the conductivity and structural stability are still obvious disadvantages of TMOs, hence the rate capability and cycling life of the electrode lag far behind those of carbon-based ones. Coupling conductive substrates like CNTs or CC has been adopted to alleviate the conductivity issues, but the intrinsic problems like metal dissolution, disproportionation reactions, and structural/phase evolution of TMOs during the charging/discharging cycles call urgently for further in-depth understandings and tackling strategies.

\subsection{Conductive polymers and emerging materials}

Conductive polymers, such as polyaniline (PANI), polypyrrole (PPy), poly(3,4-ethylenedioxythiophene) (PEDOT), poly(1,5naphthalenediamine) (PNAPD), and poly(3,3'-dihydroxybenzidine) (PDHB), have long been used as a class of pseudocapacitive materials for SCs for their high theoretical capacity and easy synthesis protocols. ${ }^{\mathbf{1 , 4 1 4 , 7 1}}$ Hence, the application of these conductive polymers has been naturally extended to ZIHCs. For example, Han et $a .^{72}$ fabricated self-supporting electrodes for ZIHCs using 3D GO@PANI composite hydrogels prepared from the in situ polymerization of aniline solution on GO. Meriting by the enlarged active interfaces of 3D structure, the hydrogel electrode exhibited a capacity of $154 \mathrm{~mA} \mathrm{~h} \mathrm{~g}^{-1}$ and a capacity retention of $80.5 \%$ after 6000 cycles in a ZIHC. Wang and co-workers ${ }^{73}$ designed a flexible sandwich-structured ZIHC cell made by combining the PDHB/AC PE and the $\mathrm{Zn}$ foil NE, which shows an areal capacitance of up to $1.3 \mathrm{~F} \mathrm{~cm}^{-2}$, and maximum energy and power densities of $0.18 \mathrm{~mW} \mathrm{~h} \mathrm{~cm}{ }^{-2}$ and $4.01 \mathrm{~mW} \mathrm{~cm} \mathrm{~cm}^{-2}$, respectively. Further miniaturization of this ZIHC still affords an areal capacitance of $1.1 \mathrm{~F} \mathrm{~cm}^{-2}$ along with flexible functions. Apart from these demonstrations, other ZIHCs based on PPy and PNAPDH have also been explored. ${ }^{74,75}$ In general, the conductive polymers exhibits charge storage performance via doping/de-doping of ions (e.g., $\left.\mathrm{Zn}^{2+}, \mathrm{H}^{+}\right)$on specific sites along the polymer chains. This unique working mechanism can cause significant volume change under prolonged cycling, resulting in a quick decrease in conductivity and mechanical strength that ultimately affects the electrochemical performance of ZIHCs. Further attempts to designing novel conducting polymers with stable physical and chemical structures are expected to improve the cycling performance.

In addition to the aforementioned $\mathrm{PE}$ materials, in recent years, some emerging materials have attracted a lot of attention in the field of energy storage for their intriguing physicochemical properties. Transition metal carbides and nitrides (MXenes) are considered as promising electrode materials that provide surficial pseudocapacitive charge storage sites together with excellent conductivity, which circumvent well the disadvantages of TMOs. ${ }^{76,77}$ Wang and colleagues ${ }^{78}$ used porous 3D MXene $\left(\mathrm{Ti}_{3} \mathrm{C}_{2} \mathrm{~T}_{x}\right)$-rGO composite aerogels as PEs for ZIHCs. To tackle the restacking of MXene nanosheets and the limited kinetics resulting from insufficient interlayer spacing, Zhi's group ${ }^{79}$ recently proposed an $\mathrm{Sn}^{4+}-\mathrm{Ti}_{2} \mathrm{CT}_{x} / \mathrm{C}$ spherical $\mathrm{PE}$ material with effectively enlarged interlayer spacing (Fig. 7a and b). The $\mathrm{Sn}^{4+}$ pre-intercalated $\mathrm{Ti}_{2} \mathrm{CT}_{x}$ was aligned on a carbon sphere to facilitate ion transportation enhancing the reaction kinetics. The GCD curves in Fig. 7c show that the potential surface barriers are significantly reduced due to the pre-embedded $\mathrm{Sn}^{4+}$. After pairing with a $\mathrm{Zn} \mathrm{NE}$, the flexible ZIHCs showed a maximum capacity of $138 \mathrm{~mA} \mathrm{~h} \mathrm{~g}^{-1}$ and a small capacity change (5\%) after 12500 cycles (equal to $2800 \mathrm{~h}$ cycle time, Fig. 7d). Also, their ZIHCs exhibited an anti-self-discharging rate of $0.989 \mathrm{mV} \mathrm{h}^{-1}$ associated with a capacity retention of $80.5 \%$ over $548 \mathrm{~h}$. The design strategies of this work may pave a new way to fabricate MXene based flexible ZIHCs.

Like MXene, phosphorene, another 2D material of great interest, has also been used in flexible quasi-solid ZIHCs. Huang et al. ${ }^{\mathbf{8 0}}$ demonstrated the first few-layered phosphorene (FL-P) based ZIHCs. The electrode material constructed from electrochemically exfoliated FL-P is stable, allowing ZIHCs to operate at relatively high voltage windows with an anti-selfdischarge capability (compared to conventional EDLC), i.e., maintaining $76.16 \%$ of the initial capacitance after $300 \mathrm{~h}$. Simultaneously, as shown in Fig. 7e-g, the electrode material can be printed on the paper-based collector by a screen printing method, enabling flexible ZIHCs with decent capacitance and good cycling stability.

So far, most emerging materials achieve charge storage through fast redox reactions. They possess good electrical conductivity and decent high specific surface areas; they can provide high specific capacitances and suppress the occurrence of self-discharge due to the formation of relatively stable chemical bonds compared to pure physical ion adsorption. Despite these merits, however, these emerging materials are 


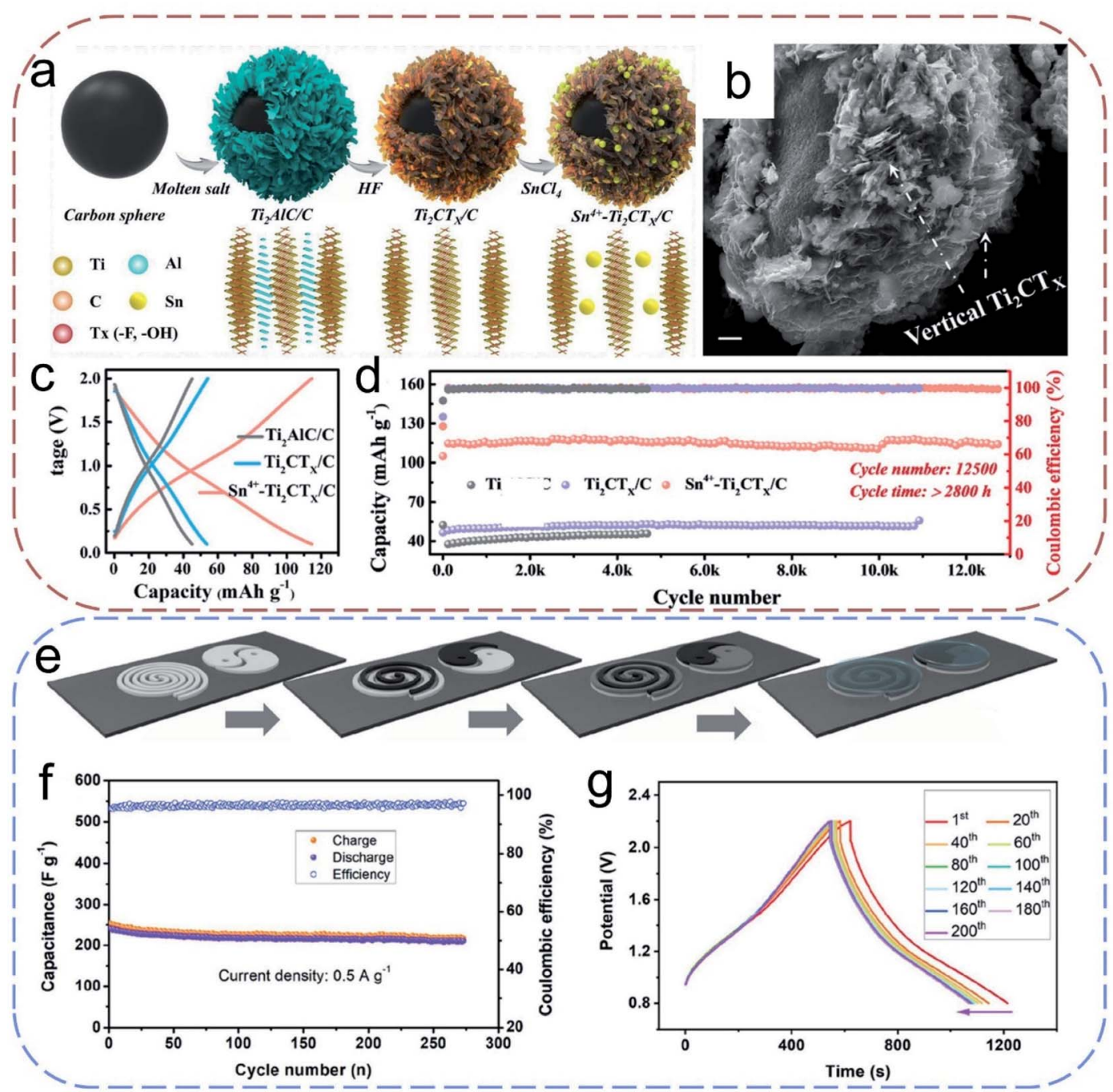

Fig. 7 (a) Schematic illustrations of the synthesis process of $\mathrm{Sn}^{4+}-\mathrm{Ti}_{2} \mathrm{CT}_{x} / \mathrm{C}$ spheres. (b) $\mathrm{SEM}$ image of $\mathrm{Sn}^{4+}-\mathrm{Ti}_{2} \mathrm{CT} \mathrm{T}_{x} / \mathrm{C}$ spheres. (c and d) $\mathrm{GCD}$ curves and cycling stability curves of $\mathrm{Ti}_{2} \mathrm{AlC} / \mathrm{C}, \mathrm{Ti}_{2} \mathrm{CT}_{x} / \mathrm{C}$, and $\mathrm{Sn}^{4+}-\mathrm{Ti}_{2} \mathrm{CT}_{x} / \mathrm{C}$ electrodes at $0.5 \mathrm{~A} \mathrm{~g}^{-1}$. Reproduced with permission. ${ }^{79} \mathrm{Copyright}$ 2020, Wiley-VCH. (e) Schematic illustrations of the electrode printing route. (f) Stability of FL-P PE constructed ZIHCs at 0.5 A g ${ }^{-1}$. (g) GCD curves of FL-P PE constructed ZIHCs at $0.5 \mathrm{~A} \mathrm{~g}^{-1}$. Reproduced with permission. ${ }^{80}$ Copyright 2020, Wiley-VCH.

suffering from easy oxidation (which leads to a quick drop in the conductivity and electrochemical performances); the synthetic procedures of these materials are still tedious, complicated, and even involve toxic reagents (e.g., hydrogen fluoride is usually used for MXenes). Thus, the development of novel electrode materials with highly efficient and stable electrochemical performances by green synthetic protocols remains one of the major challenges in the near future.

\section{Electrolytes}

\subsection{Considerations of liquid electrolytes}

For ZIHCs, the electrolyte is a pivotal component that substantially determines the electrochemical reaction behaviors at the electrode-electrolyte interfaces. Proper electrolytes should feature good ionic conductivity, low viscosity, wide voltage window, excellent temperature adaptability, and good transferability of $\mathrm{Zn}^{2+}$. For flexible devices, the interaction between the electrolyte and the polymer host needs additional consideration, and the interfacial interactions between the electrode and the electrolyte are much more complicated than those in solutions. Therefore, to better screen the electrolytes suitable for flexible ZIHCs, in-depth studies toward the electrolyte properties are mandatory.

4.1.1 Typical aqueous electrolytes. As mentioned above, the $\mathrm{KOH}$ electrolyte was first used in the proof-of-concept study of ZIHC, but it was only after the deployment of the mild $\mathrm{ZnSO}_{4}$ electrolyte that ZIHCs can afford a decent cycling stability. Since then, several mild zinc salt electrolytes, including $\mathrm{ZnSO}_{4}$, zinc chloride $\left(\mathrm{ZnCl}_{2}\right)$, zinc nitrate $\left(\mathrm{Zn}\left(\mathrm{NO}_{3}\right)_{2}\right)$, zinc acetate $\left(\mathrm{Zn}(\mathrm{Ac})_{2}\right)$, zinc triflate $\left(\mathrm{Zn}\left(\mathrm{CF}_{3} \mathrm{SO}_{3}\right)_{2}\right)$, etc., have been well-used in ZIHCs. The overall physicochemical properties of the $\mathrm{Zn}$ electrolytes change dramatically upon varying the anions; meanwhile, the properties also correlated with the electrode types. For example, 

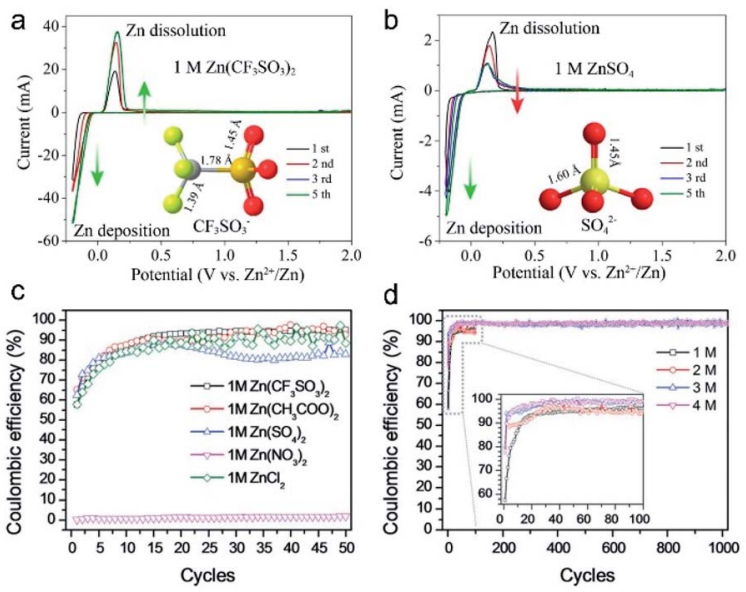

Fig. 8 (a) $\mathrm{CV}$ curves of $\mathrm{Zn}$ stripping/plating in $1 \mathrm{M} \mathrm{Zn}\left(\mathrm{CF}_{3} \mathrm{SO}_{3}\right)_{2}$. (b) $\mathrm{CV}$ curves of $\mathrm{Zn}$ stripping/plating in $1 \mathrm{M} \mathrm{ZnSO}_{4}$. Reproduced with permission. ${ }^{96}$ Copyright 2016, American Chemical Society. (c) Coulombic efficiency of Zn stripping/plating in different $Z n$ salt electrolytes. (d) Coulombic efficiency of $\mathrm{Zn}$ stripping/plating in the $\mathrm{Zn}\left(\mathrm{CF}_{3} \mathrm{SO}_{3}\right)_{2}$ electrolyte with different concentrations. Reproduced with permission. ${ }^{84}$ Copyright 2019, Wiley-VCH.
$\mathrm{Zn}\left(\mathrm{NO}_{3}\right)_{2}$ solutions, which possess strong oxidizing properties, are not suitable for ZIHCs assembled from metallic $\mathrm{Zn}$ because they rapidly oxidize metal surfaces. ${ }^{58}$ However, in Zn metal-freetype ZIHCs where other NE materials (e.g., carbon materials) are used, the $\mathrm{Zn}\left(\mathrm{NO}_{3}\right)_{2}$ electrolytes can still enable satisfactory electrochemical performances. ${ }^{\mathbf{9 0}}$ In this sense, the materials of the electrode are also the prerequisite for selecting these mild $\mathrm{Zn}$ electrolytes. In most existing studies, the choice of the electrolyte is based on the assumption that $\mathrm{Zn}$ metal is the $\mathrm{NE}$, hence $\mathrm{ZnSO}_{4}$ and $\mathrm{Zn}\left(\mathrm{CF}_{3} \mathrm{SO}_{3}\right)_{2}$ are the two most widely used zinc salts. In particular, $\mathrm{Zn}\left(\mathrm{CF}_{3} \mathrm{SO}_{3}\right)_{2}$ electrolytes enable $\mathrm{Zn}$ with the highest and most stable Coulombic efficiency for the plating/ stripping process (Fig. 8). ${ }^{\mathbf{8 4}, 96}$ An optimal $\mathrm{Zn}\left(\mathrm{CF}_{3} \mathrm{SO}_{3}\right)_{2}$ concentration of 3 or $4 \mathrm{M}$ (molar per liter solution) can not only render average Coulombic efficiencies of $\sim 99 \%$ for $\mathrm{Zn}$ plating/ stripping but also enhance the stability of $\mathrm{PE}$ materials. However, the price of $\mathrm{Zn}\left(\mathrm{CF}_{3} \mathrm{SO}_{3}\right)_{2}$ is too high compared to other zinc salts (about 50 times more expensive than $\mathrm{ZnSO}_{4}$ and $\mathrm{ZnCl}_{2}$ ), which will raise the overall cost of the whole ZIHCs.

The above pioneering works undeniably underpin the understanding from the NE perspective; however, conclusions may differ when considering the PE materials. Recently, Qu's group ${ }^{90}$ first systematically investigated the effects of different a

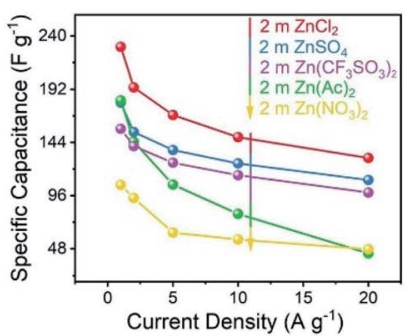

d
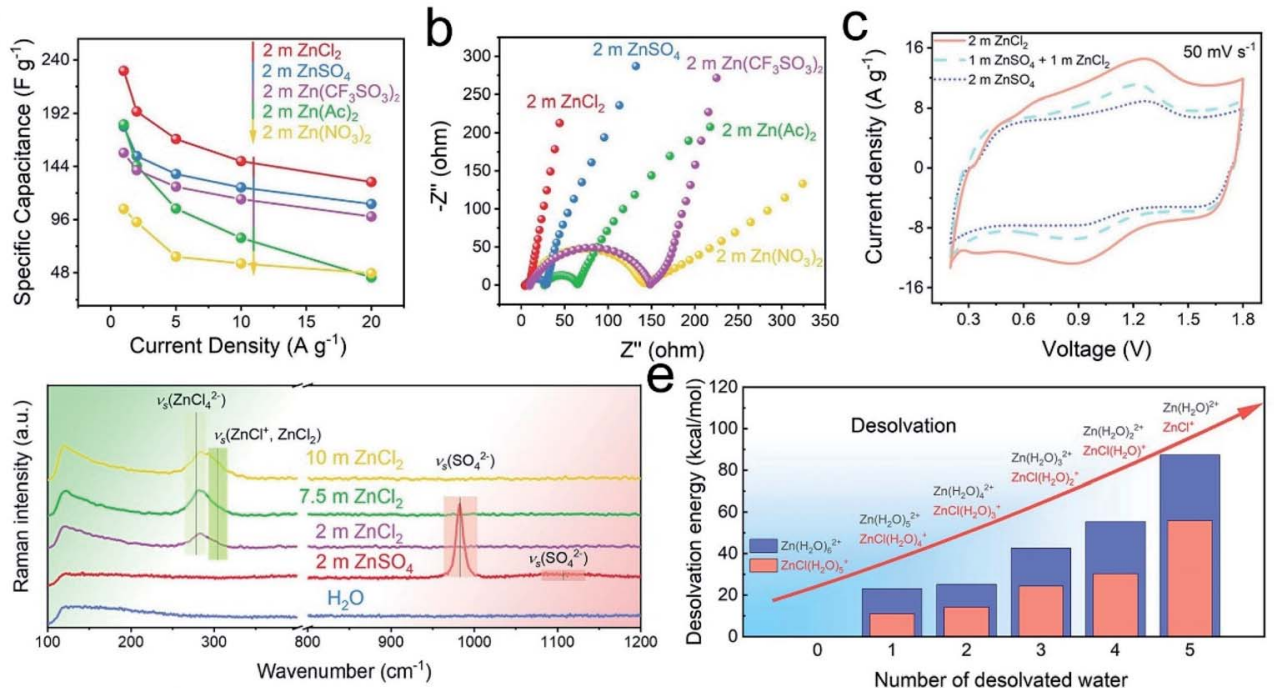

e

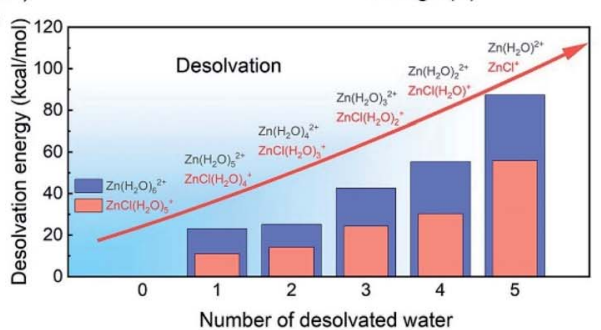

f

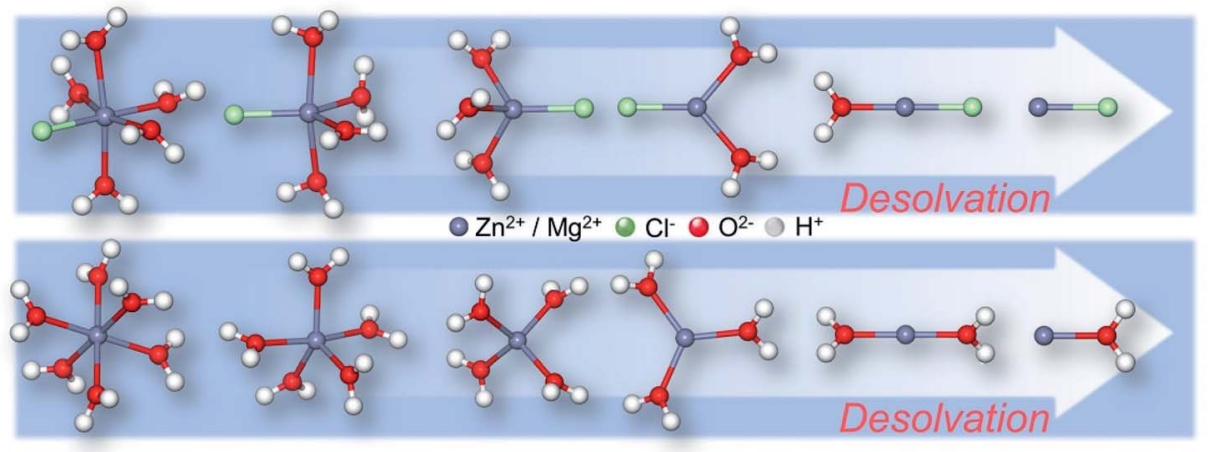

Fig. 9 (a) Rate capability of ZIHCs in different zinc salt solutions. (b) Nyquist curves. (c) CV curves of ZIHCs at $50 \mathrm{mV} \mathrm{s}{ }^{-1}$. (d) Raman spectra of different solutions. (e) Desolvation energies of $\left[\mathrm{ZnCl}\left(\mathrm{H}_{2} \mathrm{O}\right)_{5}\right]^{+}$and $\left[\mathrm{Zn}\left(\mathrm{H}_{2} \mathrm{O}\right)_{6}\right]^{2+}$. (f) Molecular geometries of the $\left[\mathrm{ZnCl}\left(\mathrm{H}_{2} \mathrm{O}\right)_{5}\right]^{+}$and $\left[\mathrm{Zn}\left(\mathrm{H}_{2} \mathrm{O}\right)_{6}\right]^{2+}$ desolvation processes. Reproduced with permission. ${ }^{58}$ Copyright 2020, Wiley- $\mathrm{VCH}$. 
electrolytes on the energy storage performance of carbon PE materials through combined theoretical calculations and in situ electrochemical analyses. They corroborated that the hydrated metal ion undergoes a partial desolvation process during the ion storage process at PE. The partial desolvation process was then leveraged to increase the spatial charge density (SCD) of PE by balancing the valence and the size of charge-carrier ions and matching the ion sizes with the pore structure of electrode materials. As a proof-of-concept demonstration, a record-high SCD of about $550 \mathrm{C} \mathrm{cm}^{-3}$ was achieved by pairing the $\mathrm{Zn}^{2+}$ ions with a highly ordered and compact porous carbon in a ZIHC. This work lays a milestone in understanding the influence of electrolytes on PE energy storage. Wang et al. ${ }^{58}$ then systematically studied the influence of a series of typical anions on the desolvation process of $\mathrm{Zn}^{2+}$ ions and proved that the presence of $\mathrm{Cl}^{-}$species can significantly improve the energy density of AC electrodes (Fig. 9a-c). Note that in other aqueous $\mathrm{Zn}$ salt electrolytes, such as $\mathrm{ZnSO}_{4}$ and $\mathrm{Zn}\left(\mathrm{CF}_{3} \mathrm{SO}_{3}\right)_{2}$, $\mathrm{Zn}$ species generally exist as $\left[\mathrm{Zn}\left(\mathrm{H}_{2} \mathrm{O}\right)_{6}\right]^{2+}$, whose radius $(8.60 \AA)$ is much larger than that of the bare $\mathrm{Zn}^{2+}$ ion $(1.48 \AA) .{ }^{97-99}$ In this work, through combined elaborated experimental characterizations and DFT calculations, the authors unraveled for the first time that the desolvation energy of $\mathrm{Cl}^{-}$-induced $[\mathrm{ZnCl}]^{+}\left(\mathrm{H}_{2} \mathrm{O}\right)_{n-1}$ (with $n=1-6$ ) clusters is significantly lower than its $\left[\mathrm{Zn}\left(\mathrm{H}_{2} \mathrm{O}\right)_{n}\right]^{2+}$ counterparts, hence the smaller $[\mathrm{ZnCl}]^{+}\left(\mathrm{H}_{2} \mathrm{O}\right)_{n-1}$ clusters can easily enter otherwise un-accessible micropores of the AC to remarkably improve the storage capacity of AC (Fig. 9d-f). Such an intriguing $\mathrm{Cl}^{-}$-facilitated desolvation mechanism in hydrated $[\mathrm{ZnCl}]^{+}\left(\mathrm{H}_{2} \mathrm{O}\right)_{n-1}$ (with $\left.n=1-6\right)$ clusters opens up a new way to enhance the energy density of PC materials for ZIHCs. More generally, the mechanism was demonstrated in hydrogel electrolytes and analogous $\mathrm{Mg}^{2+}$ salt electrolytes. Based on these findings, the authors further developed a $\mathrm{ZnCl}_{2}$ based "water-in-salt" type hydrogel electrolyte, which enables the flexible ZIHCs to deliver a battery-level energy density (up to $217 \mathrm{~W} \mathrm{~h} \mathrm{~kg}^{-1}$ at a power density of $450 \mathrm{~W}$ $\mathrm{kg}^{-1}$, based on carbon materials) and unprecedented cycling life with $95.1 \%$ capacity retention after 100000 charging/ discharging cycles.

Aside from the influence on the desolvation of hydrated $\mathrm{Zn}^{2+}$ ions, anions of $\mathrm{Zn}$ salt solutions affect the charge storage capability of other PE materials. Recently, Zhi's group ${ }^{91}$ found that in a $\mathrm{Zn//titanium} \mathrm{nitride} \mathrm{(TiN)} \mathrm{based} \mathrm{ZIHC,} \mathrm{the} \mathrm{anions} \mathrm{have}$ a substantial impact on the TiN PE's capacity/capacitance and

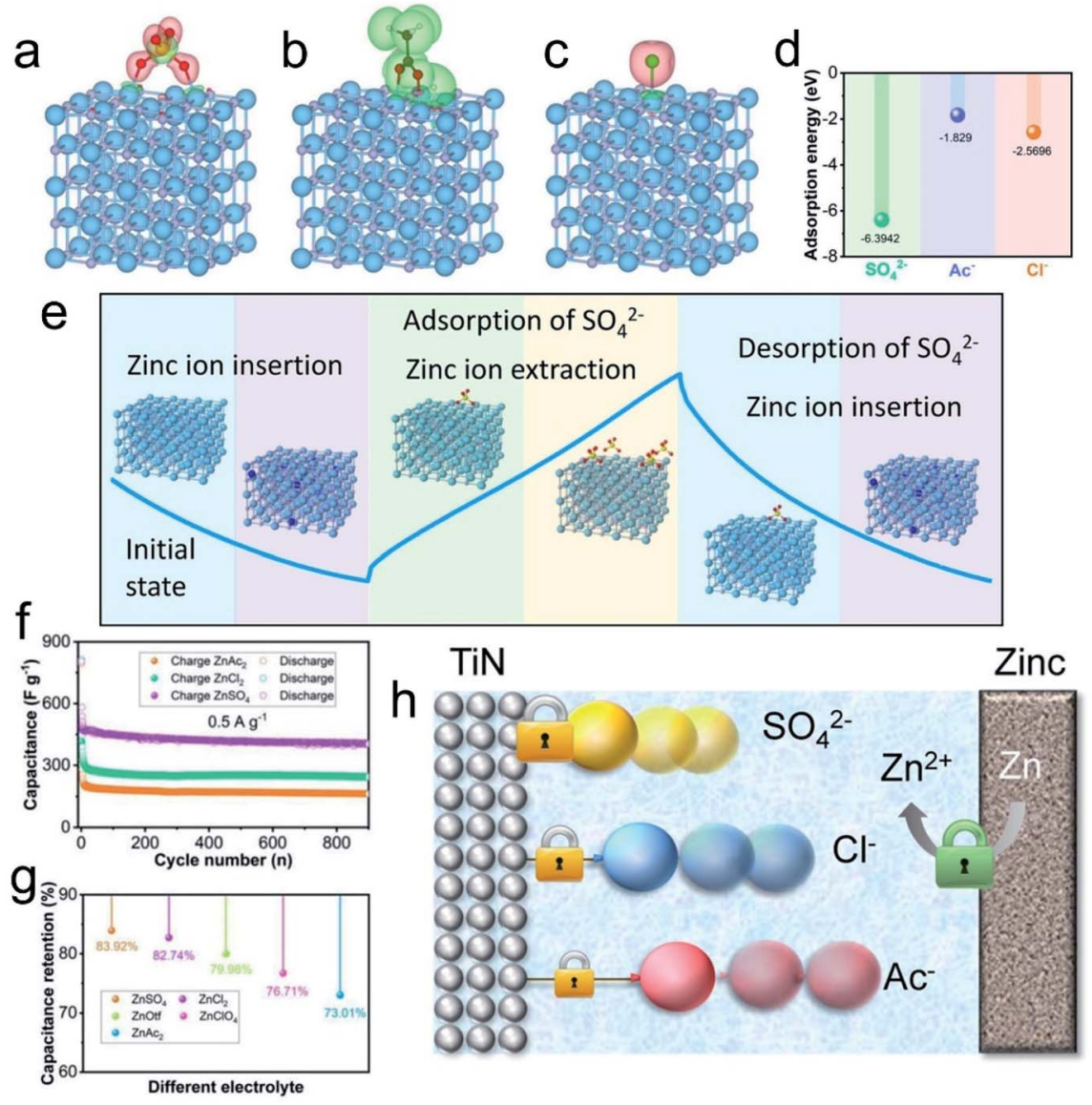

Fig. 10 (a) Charge-density differences after the adsorption of anions $\mathrm{SO}_{4}{ }^{2-}$, (b) $\mathrm{Ac}^{-}$, and (c) $\mathrm{Cl}^{-}$on the (110) lattice plane of TiN. (d) Adsorption energy of TiN for different anions. (e) Energy storage mechanism during the charging/discharging process. (f) Cycling performance of Zn-TiN ZIHCs with different electrolytes. (g) The corresponding capacitance retention rate after the self-discharge test. (h) Schematic diagram of the difference in the self-discharge behavior of ZIHCs with different electrolytes. Reproduced with permission. ${ }^{91} \mathrm{Copyright} 2020, \mathrm{Wiley}-\mathrm{VCH}$. 
anti-self-discharging capability. DFT calculations show that, among three typical anions of $\mathrm{SO}_{4}{ }^{2-}, \mathrm{Ac}^{-}$, and $\mathrm{Cl}^{-}, \mathrm{SO}_{4}{ }^{2-}$ ions have the highest adsorption energy on TiN (Fig. 10a-d), indicating that $\mathrm{SO}_{4}{ }^{2-}$ ions are more easily adsorbed onto TiN and have the most stable structure after adsorption. They also proved that $\mathrm{SO}_{4}{ }^{2-}$ ions participate in the charging/discharging process through a two-step adsorption and intercalation energy storage mechanism: $\mathrm{SO}_{4}{ }^{2-}$ ion desorption and $\mathrm{Zn}^{2+}$ ion insertion in discharge, with the converse process in charge (Fig. 10e). Due to the capacitance effect of $\mathrm{SO}_{4}{ }^{2-}$ ions, the constructed ZIHCs have a larger capacitance over the other two counterparts (Fig. 10f). Meanwhile, meriting by the more stable structure of $\mathrm{SO}_{4}{ }^{2-}$ ions formed with TiN during adsorption, $\mathrm{ZnSO}_{4}$ based ZIHCs have the best self-discharging resistance $(83.9 \%$ after $500 \mathrm{~h}$ ) over all other anions (Fig. $10 \mathrm{~g}$ and $\mathrm{h}$ ). Their work highlights again that the type of electrode is the key factor when considering the effect of anions on the overall energy storage performances of ZIHCs.

To sum up, different anions can greatly impact the solvation structure of $\mathrm{Zn}^{2+}$; the large radius of hydrated $\mathrm{Zn}$ ions limits the SCD of the electrode materials. Therefore, anions that can modify the solvation sheath of $\mathrm{Zn}$ ions are very attractive because they can change the desolvation energy of the solvated $\mathrm{Zn}$ clusters to potentially increase the energy density of the devices. However, the choice of anions is determined by both the PEs and NEs, hence the solvation structures and transport behaviors of $\mathrm{Zn}$ ions in various electrolytes should be further investigated.

4.1.2 Organic electrolytes and ionic liquids. Although aqueous electrolytes are widely used, one major hurdle is their narrow voltage window caused by water decomposition. To this end, organic electrolytes and ionic liquids have been studied. Organic solvents, such as acetonitrile (AN), dimethoxyethane (DME), and 1,3-dioxolane (DOL), are common solvents in organic electrolytes for $\mathrm{ZIHCs}$, whilst $\mathrm{Zn}\left(\mathrm{CF}_{3} \mathrm{SO}_{3}\right)_{2}$ is mostly used as a solute because of its good solubility in many organic solutions. Tang's group ${ }^{85}$ reported the first organic electrolyte for ZIHCs by dissolving $1 \mathrm{M} \mathrm{Zn}\left(\mathrm{CF}_{3} \mathrm{SO}_{3}\right)_{2}$ in $\mathrm{AN}$, which can be worked in a voltage range of $0-1.8 \mathrm{~V}$. Owing to the eliminated side reactions, the capacitor achieved a high Coulombic efficiency close to $100 \%$ and an energy density of $52.7 \mathrm{~W} \mathrm{~h} \mathrm{~kg}^{-1}$ at a power density of $1725 \mathrm{~W} \mathrm{~kg}^{-1}$ (based on carbon). However, the rate capability of the ZIHC was moderate because of the low conductivity of the electrolyte. Similar to organic electrolytes, ionic liquids have emerged as a new option for anhydrous or waterless electrolytes. Zhou and colleagues ${ }^{86}$ used an ionic liquid $\left(\mathrm{EMIMCF}_{3} \mathrm{SO}_{3}\right)$ as a solvent that improved the range of operating voltages compared with that of AN, enabling the constructed ZIHCs to operate stably up to $2.4 \mathrm{~V}$. The optimal ZHIC can achieve a specific capacitance of $198 \mathrm{~F} \mathrm{~g}^{-1}$ at $0.2 \mathrm{~A} \mathrm{~g}^{-1}$ and an energy density of $54.3 \mathrm{~W} \mathrm{~h} \mathrm{~L}^{-1}$, comparable to that of lead-acid batteries. It should be noted that ionic liquids can also bind to aqueous solutions, resulting in reduced free water in the solution. Liu et al. ${ }^{100}$ developed a biodegradable ionic liquid-based electrolyte using $70 \%$ choline acetate ([Ch]OAc) and $30 \%$ water as the solvent and $\mathrm{Zn}(\mathrm{Ac})_{2}$ as the solute. The authors demonstrated via Raman spectroscopy that ionic liquids can influence the formation of coordination complexes of $\mathrm{Zn}^{2+}$ ions, forming complexes of $\left[\mathrm{Zn}(\mathrm{OAc})_{4}\right]^{2-}$ which improved the thermodynamic and kinetic processes of $\mathrm{Zn}$ deposition. Despite these encouraging signs of progress, the low conductivity of both organic and ionic liquid electrolytes will always render mediocre rate capability in ZIHCs. More critically, intrinsic safety concerns (e.g., flammability, toxicity) of the organic electrolytes are another major barrier limiting their usage in flexible ZIHCs.

4.1.3 Water-in-salt electrolytes. Since Xu and Wang's pioneering work in 2015, ${ }^{101}$ the water-in-salt (WIS) electrolyte has a

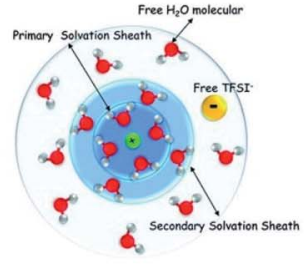

$\left\{\left[\mathrm{Li}\left(\mathrm{H}_{2} \mathrm{O}\right)_{4}\right]\left(\mathrm{H}_{2} \mathrm{O}\right)_{4}\right\}+\mathrm{nH}_{2} \mathrm{O} \mathrm{n} \geq 1$ Salt-in-Water

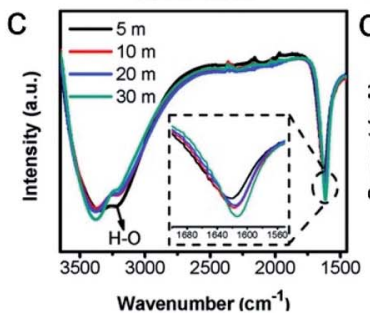

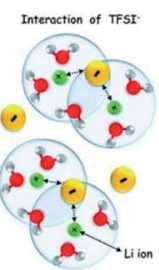

$\mathrm{Li}\left(\mathrm{H}_{2} \mathrm{O}\right)_{2.5}$-TFSI Water-in-Salt
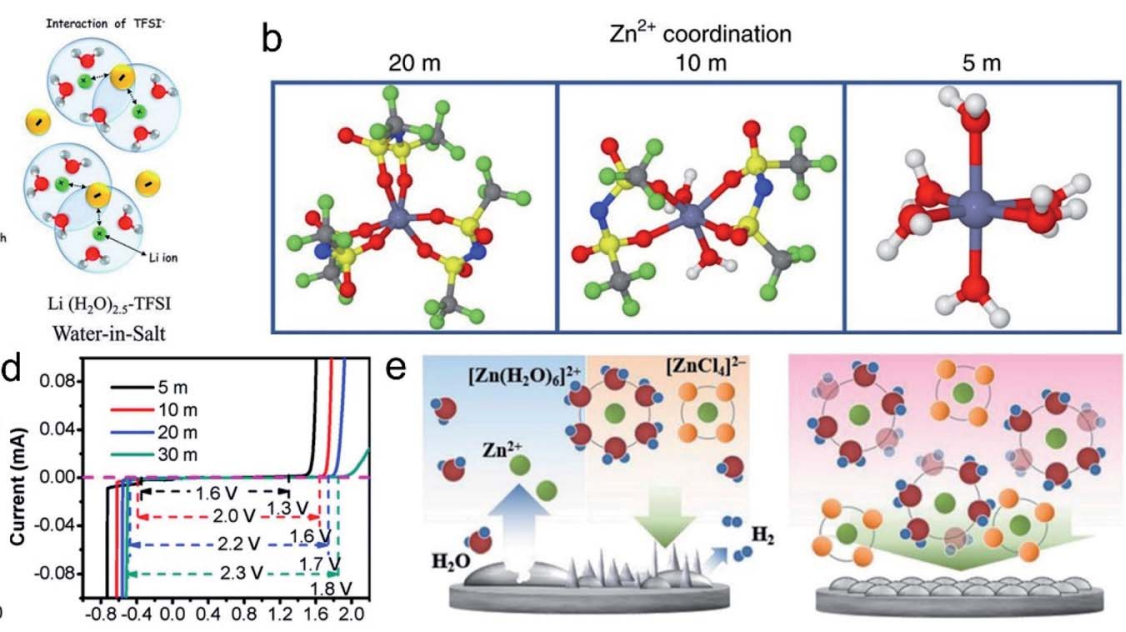

Voltage (V vs SHE)

Fig. 11 (a) Primary solvation sheath of $\mathrm{Li}^{+}$in dilute and WIS-type LiTFSI electrolytes. Reproduced with permission. ${ }^{101}$ Copyright 2015 , The American Association for the Advancement of Science. (b) Primary solvation sheath of $\mathrm{Zn}^{2+}$ in LiTFSI electrolytes with different concentrations. Reproduced with permission. ${ }^{102}$ (c) FTIR spectra of $\mathrm{ZnCl}_{2}$ electrolytes with different concentrations. (d) The corresponding electrochemical window. ${ }^{103}$ Copyright 2018, Springer Nature. (e) Hydrogen evolution, Zn dendrite growth, and shape change issues in dilute and WIS-type $\mathrm{ZnCl} 2$ electrolytes. Reproduced with permission. ${ }^{99}$ Copyright 2019, Wiley-VCH. 
become one of the most popular electrolyte types in the energy storage field because it provides novel properties such as the expanded potential window and enhanced Coulombic efficiency for batteries and SCs. The WIS electrolyte is defined as the weight and volume of salt that exceeds that of water in a binary system, and the term molality ( $\mathrm{m}, 1 \mathrm{~m}$ means 1 mol solute dissolved in per kilogram of deionized water) is frequently used to describe the concentration of the salt in WIS electrolytes. The ultra-high concentration of WIS enables the water molecules to exist in the hydrated state rather than in the free state as in the solution. In turn, the $\mathrm{O}-\mathrm{H}$ bond in the water molecule is enhanced, and the water molecule becomes difficult to be electrolyzed because most of the water molecules form a solvation shell around the metal cations (Fig. 11a). Later, Xu and Wang further demonstrated that using a WIS electrolyte consisting of $1 \mathrm{~m} \mathrm{Zn(TFSI})_{2}+20 \mathrm{~m}$ LiTFSI can effectively inhibit the growth of $\mathrm{Zn}$ dendrites. ${ }^{\mathbf{1 0 2}}$ Molecular dynamics simulation results suggest that the $\mathrm{Zn}^{2+}$ ions possess a unique solvation sheath in the optimized WIS electrolyte $(20 \mathrm{~m})$, where the abundant $\mathrm{TFSI}^{-}$anions force them into the vicinity of $\mathrm{Zn}^{2+}$ to form $\mathrm{Zn}$-TFSI ion pairs (Fig. 11b). The water molecules are fixed so that parasitic HER and formation of byproducts were suppressed during $\mathrm{Zn}$ plating/stripping, which eventually leads to the high Coulombic efficiency and dendrite-free $\mathrm{Zn}$ deposition. Following this, the two advantages have become an important basis for the development of WIS electrolytes in ZIHCs.

Though effective, one notorious drawback of LiTFSI is its very high cost. Therefore, other salts with high solubility and lower price have been pursued by researchers. However, the conceivable "simple" $\mathrm{Zn}$ salts that can venture into the WIS regime have been limited, hitherto, to $\mathrm{ZnCl}_{2}$ exclusively (its concentration in water can be as high as $31 \mathrm{~m}$ ). Ji's group ${ }^{\mathbf{1 0 3}}$ first proposed the use of $\mathrm{ZnCl}_{2}$ with ultra-high solubility to prepare the WIS electrolyte, which indeed enables both a high potential window and effective $\mathrm{Zn}$ dendrite suppression. They found that increasing the $\mathrm{ZnCl}_{2}$ concentration significantly affects the $\mathrm{O}-\mathrm{H}$ bonding to reduce the side reactions, and a wide electrochemical window of $2.3 \mathrm{~V}$ can be obtained in the $30 \mathrm{~m} \mathrm{ZnCl}_{2}$ electrolyte (Fig. 11c and d). Moreover, the minimal amount of free water forces the reduction of water molecules in the solvent shell of $\mathrm{Zn}^{2+}$ ions, while anionic groups of $\left(\mathrm{ZnCl}_{4}\right)^{2-}$ and $\left[\mathrm{Zn}\left(\mathrm{OH}_{2}\right)_{2} \mathrm{Cl}_{4}\right]^{2-}$ are more easily formed instead of $\mathrm{Zn}\left(\mathrm{H}_{2} \mathrm{O}\right)_{6}{ }^{2+}$. Subsequently, Xu's group ${ }^{99}$ revealed that $\left(\mathrm{ZnCl}_{4}\right)^{2-}$ increases significantly with increasing concentration of $\mathrm{ZnCl}_{2}$ and that the hyper-concentrated $\mathrm{ZnCl}_{2}$ solution is closer to those of ionic liquids, thus the WIS electrolyte can also suppress common problems like HER, Zn dendrites, and shape change in dilute solutions (Fig. 11e). However, one non-negligible problem associated with $\mathrm{ZnCl}_{2}$ electrolytes is that $\mathrm{Cl}^{-}$can be oxidized at high voltages, and the possible release of $\mathrm{Cl}_{2}$ deserves particular care.

In essence, while different salt species may result in specific solvation sheath structures, the advantages of WIS electrolytes are similar and general. The success of the $\mathrm{ZnCl}_{2}$ WIS electrolyte implies that other salts with high solubility (e.g., sodium perchlorate $\left(\mathrm{NaClO}_{4}\right)$, potassium acetate (KAc)) can afford similar properties as long as they can reach a high concentration required by the WIS definition. Very recently, Lu's group ${ }^{\mathbf{1 0 4}}$ extended the WIS electrolyte by proposing a "molecular crowding" aqueous electrolyte via confining water molecules in a poly(ethylene glycol) (PEG) network crowding agent through hydrogen bonding. Such a novel electrolyte deploys a low salt concentration ( $2 \mathrm{~m}$ LiTFSI) yet achieves a high operational cell voltage of 3.2 V. Their work provides a very promising path for designing low-cost, eco-friendly, and high-voltage aqueous electrolytes that liberate the requirement for ultra-high solubility salts, thus holding great prospects for ZIHCs. Nevertheless, there is a trade-off between the concentration and ionic conductivity of WIS electrolytes; and the water to salt ratio should be balanced to achieve a satisfying electrochemical performance in ZIHCs. When applied in flexible devices, the compatibility between the WIS electrolyte and the polymer matrix is another challenge, which will be discussed below.

\subsection{Gel polymer electrolytes}

With the increasing demand for flexible functionalities, gel polymer electrolytes (GPEs) have aroused intensive research interest in Zn-based energy storage devices. In flexible ZIHCs, the GPEs not only work as an electrolyte to conduct charges between PE and NE but also serve as a separator to provide ionic movement channels. In principle, a competent GPE for ZIHCs should present some typical features: (1) high ionic conductivity and $\mathrm{Zn}^{2+}$ ion transfer number; (2) a low glass transition temperature $\left(T_{\mathrm{g}}\right)$; (3) satisfying salt-dissociation capability; and (4) mechanical and (electro)chemical stability. ${ }^{105}$ To meet these requirements and provide good electrochemical/mechanical properties for the whole device, the types of gel backbone, terminal functional groups, electrolyte species and concentration, and the crosslinking conditions in GPEs need overall considerations. Fig. 12 shows the molecular structures of some typically used polymers for GPEs.

Currently, one main challenge of GPEs is their greatly compromised ionic conductivity (compared with that of liquid equivalent), which limits the desired power density of ZIHCs at large current densities. For example, although widely used, the

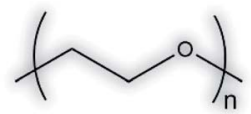

PEO

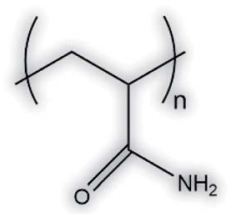

PAM

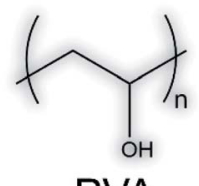

PVA

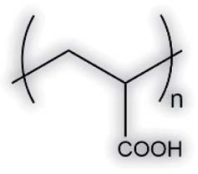

PAA

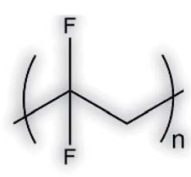

$\mathrm{PVdF}$

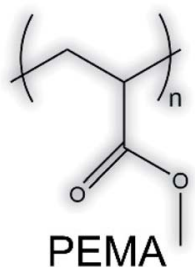

Fig. 12 Molecular structures of some typically used polymers for GPEs. 
ionic conductivity of polyethylene oxide (PEO), ${ }^{\mathbf{1 0 6}, \mathbf{1 0 7}}$ polyvinyl alcohol (PVA), ${ }^{38,51,57,108}$ and gelatin ${ }^{50,62,67,94,109,110}$ based electrolytes is in the range of $10^{-5}$ to $10^{-3} \mathrm{~S} \mathrm{~cm}^{-1}$. Cross-linked polyacrylamide (PAM) and polyacrylic acid (PAA) based GPEs are reported to exhibit higher high conductivity (tens of $\mathrm{mS} \mathrm{cm}^{-1}$ at room temperature $)^{52,55}$ because the high content of trapped gel water can expedite the drift of dissolved ions. Generally, GPEs with more polarized terminal groups and/or interconnected pores can host more water, which is beneficial for improved conductivity. However, excess water will inevitably deteriorate the mechanical strength of GPEs, hence a delicate balance toward the trade-off between water content and mechanical properties needs careful optimization.

On the other hand, the interaction between the zinc salt and polymer backbones plays a non-negligible role in both the electrochemical and mechanical properties of GPEs. ${ }^{\mathbf{1 1 1}, 112}$ For example, by evaluating PVA-based GPEs with various $\mathrm{Zn}$ salts (including $\mathrm{ZnSO}_{4}, \mathrm{ZnCl}_{2}$, and $\left.\mathrm{Zn}\left(\mathrm{CF}_{3} \mathrm{SO}_{3}\right)_{2}\right)$, Ma's group ${ }^{113}$ found that the ion conductivity based on small-anion salts $\left(\mathrm{ZnCl}_{2}\right.$ and $\left.\mathrm{ZnSO}_{4}\right)$ was higher than that of large-anion salts $\left(\mathrm{Zn}\left(\mathrm{CF}_{3} \mathrm{SO}_{3}\right)_{2}\right)$. Wang and co-authors ${ }^{58}$ reported that a typical cross-linked PAMbased GPE could afford a peak conductivity of $71.4 \mathrm{mS} \mathrm{cm}^{-1}$ upon accommodating $\mathrm{ZnCl}_{2}$ with an optimal concentration of $7.5 \mathrm{~m}$; a further increase of the salt concentration will stiffen GPEs with limited stretchability and conductivity. Such results are presumably caused by the hygroscopic nature of $\mathrm{ZnCl}_{2}$, which can extract water from the PAM matrix. In another work, PAM was reported to accommodate $21 \mathrm{~m}$ LiTFSI and $1 \mathrm{~m}$ $\mathrm{Zn}\left(\mathrm{CF}_{3} \mathrm{SO}_{3}\right)_{2}$ salts while maintaining the electrochemical properties and flexibility. ${ }^{80}$ To utilize the merits of WIS electrolytes, it is still challenging to develop hydrogels that are resistant to selfshrinkage in divalent cationic salts while maintaining good electrochemical and mechanical properties. Recently, He's group systematically studied the Hofmeister effect of several salts in PVA hydrogels. ${ }^{114}$ Their work may inspire the rational design of GPEs for flexible ZIHCs.

Note that GPEs consist of a single type of polymer that can hardly satisfy all the requirements, composite GPEs are therefore developed to combine the complementary advantage of each component. Co-polymerization of two or more monomers and cross-linking different polymers (via hydrogel bonds or chemical crosslinkers) are two general ways to synthesize composite GPEs. For example, considering that ion transfer occurs in the amorphous region of the polymer, ${ }^{115,116} \mathrm{Wu}$ et al. showed that cross-linking of PAA with PVA can increase the amorphous phase's proportion within the composite GPE to increase its overall ionic conductivity. ${ }^{117}$ Han et al. ${ }^{118}$ reported a GPE that co-crosslinks PAA with PAM via sodium alginate (SA), which delivered a high conductivity of $28.2 \mathrm{mS} \mathrm{cm}^{-1}$ together with an impressive stretchability with an elongation of $1700 \%$ under 0.13 MPa stress.

In the last few years, the rejuvenation of aqueous $\mathrm{Zn}$ batteries has stimulated substantial studies on GPEs to satisfy the everincreasing demand for flexible and wearable electronics. Many of the GPEs can be naturally extended to ZIHCs. In general, one overarching challenge for flexible devices is the poor wettability between the electrolyte-electrode interfaces. Hence, with the basic requirements of ionic conductivity and mechanical properties, further efforts should be reasonably devoted to enhancing the interfacial contact and understanding the interfacial properties. Moreover, shape integrity is another metric that is vital for the long-term usage of flexible ZIHCs.

\section{NEs for flexible zinc ion hybrid capacitors}

The NE materials commonly used in flexible ZIHCs can be divided into two types: $\mathrm{Zn}$ metal materials and $\mathrm{Zn}$ metal-free (or termed as "rocking-chair" type) ones. Among them, Zn metal NEs have drawn overwhelming attention due to their merits of high electrical conductivity, high natural abundance, a relatively low redox potential of $-0.76 \mathrm{~V}$ ( $v s$. SHE), and high volumetric energy density (which is particularly suitable for flexible ZIHCs). ${ }^{38,79,80,91}$ However, the implementation of $\mathrm{Zn}$ NEs in aqueous electrolytes is restricted by bottlenecks of poor cycling life and limited Coulombic efficiency, which typically stem from several notorious problems including the dendrite growth, corrosion, and passivation (see the schematic in Fig. 13). Though organic electrolytes can suppress water-induced problems, they are not suitable for flexible ZIHCs because of both performance limitation (e.g., poor power density) and safety concerns (i.e., toxicity and flammability). Alternatively, deploying "rocking-chair" type NE electrodes is another viable solution to retard the aforementioned challenges, but the overall energy

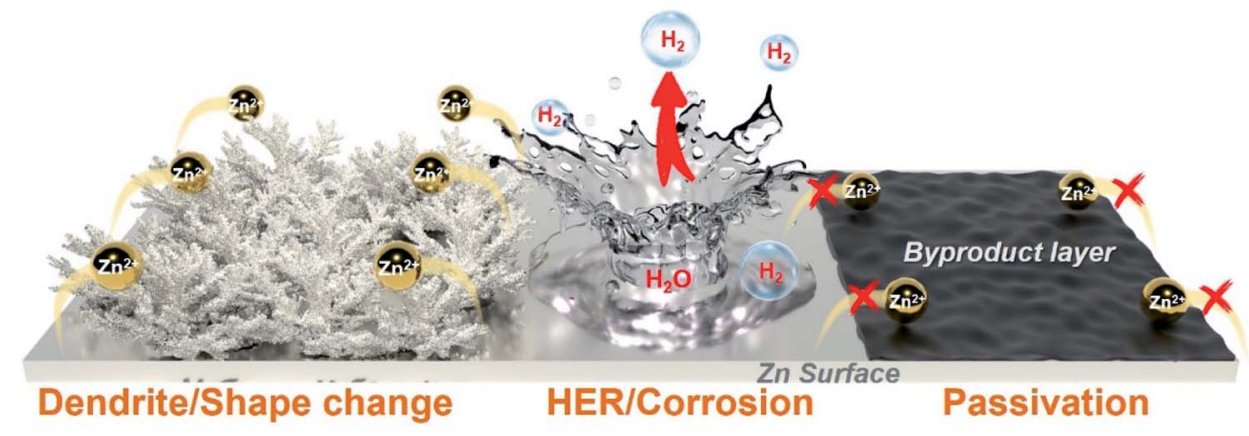

Fig. 13 Schematic diagram showing the dendrite/shape change, HER/corrosion, and passivation problems of Zn NEs. 
densities of the ZIHC devices can be seriously compromised at the same time. In view of flexible ZIHCs, most existing research studies deploy Zn foils as NEs, presumably because the planar foil is convenient for experimental observations. However, planar metal foils are almost impossible for eventual devices because of their rigidity and limited electrochemical surface areas. Lacking flexible $\mathrm{Zn}$ electrodes is another key challenge to fulfill the "flexible prospects" depicted by flexible ZIHCs. In this section, we summarize the latest feasible strategies towards all these challenges in developing NEs for ZIHCs.

\subsection{Zn metal electrodes}

The reaction mechanism of $\mathrm{Zn}$ metal NEs in ZIHCs is the reversible plating/stripping of $\mathrm{Zn}^{2+}$ on the electrode surface. Ideally, $\mathrm{Zn}^{2+}$ ions should be evenly deposited on the surface of Zn NE with a uniform ion flux driven by a uniform electric field. However, such an ideal state is almost impossible because there are inevitable irregular domains on the $\mathrm{Zn}$ metal surface (from the manufacturing process) and localized $\mathrm{Zn}^{2+}$ ion concentration gradients. Hence, practical Zn plating/stripping is always inhomogeneous, which aggravates the irregular surface appearance and causes the subsequent growth of $\mathrm{Zn}$ dendrites, known as the "tip effect". ${ }^{119}$ With repeated plating/stripping processes, the local electric field and the ion flux at the interface become more non-uniform, which further exacerbates the sharpness of dendrites until it eventually punctures separators and causes an irreversible short circuit of the devices. ${ }^{\mathbf{1 2 0 , 1 2 1}}$ of equal importance are the incessant faradaic and non-faradaic side reactions that are also the "Achilles heel" for real reversible Zn NEs. ${ }^{122}$ According to the Pourbaix diagram, metallic Zn is thermodynamically unstable even in neutral and mildly acidic electrolytes, leading to corrosion and HER. These competing side reactions not only consume $\mathrm{Zn}$ and electrolyte but also increase the internal pressure (even explosion) of ZIHCs. Furthermore, the insulating byproducts (e.g., $\left.\mathrm{Zn}(\mathrm{OH})_{x}\right)$ formed during plating/stripping cycles will passivate the $\mathrm{Zn}$

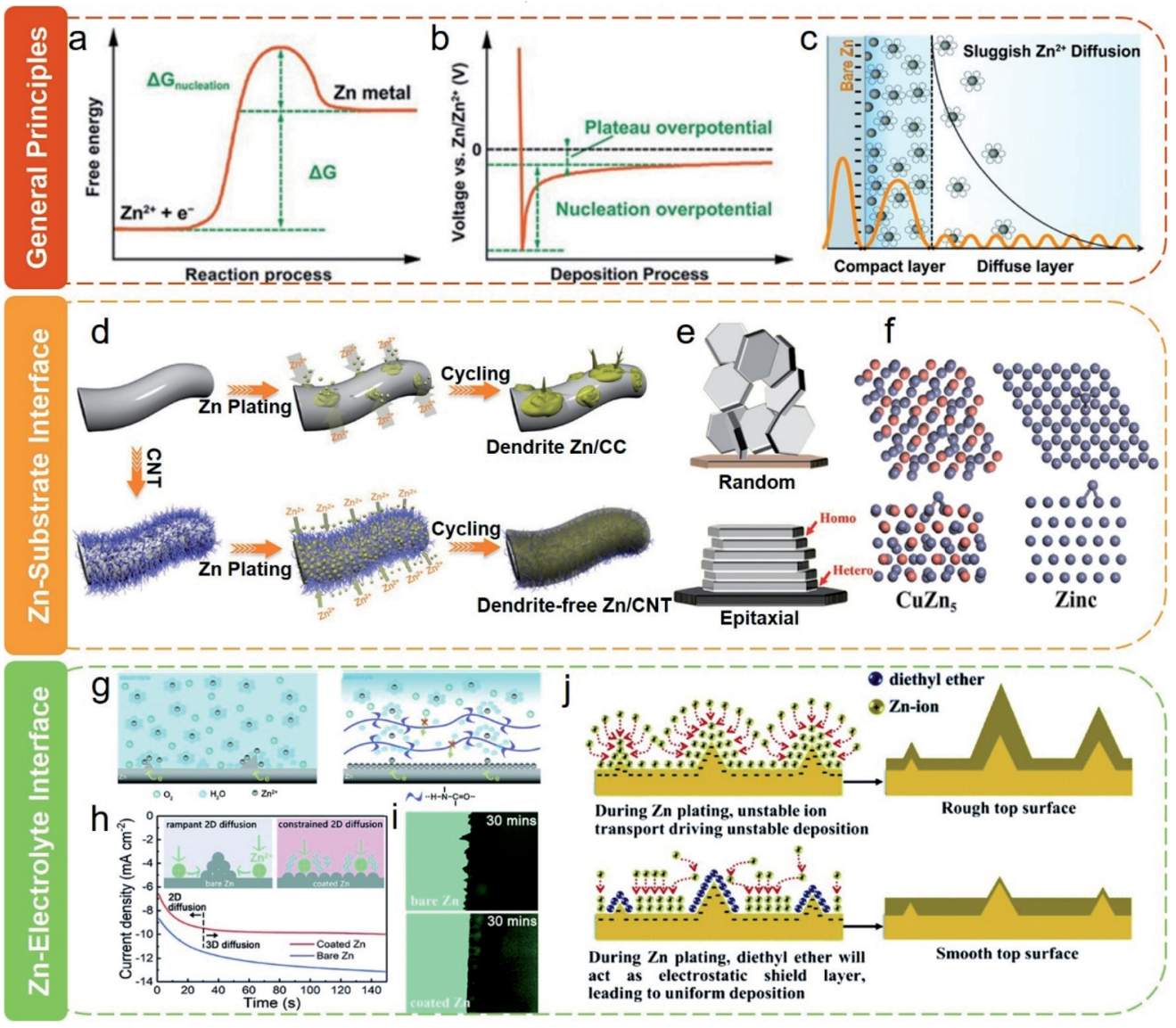

Fig. 14 (a) Energy barrier in the process of Zn nucleation and (b) typical voltage curve during Zn deposition. Reproduced with permission. ${ }^{127}$ Copyright 2019, Wiley-VCH. (c) Electric double-layer model for bare Zn. Reproduced with permission. ${ }^{129}$ Copyright 2020 , The Royal Society of Chemistry. (d) Zn deposition on carbon cloth (CC) and carbon nanotube (CNT) substrates. Reproduced with permission. ${ }^{130}$ Copyright 2019 , Wiley-VCH. (e) Random and epitaxial electrodeposition. Reproduced with permission. ${ }^{131}$ Copyright 2019, The American Association for the Advancement of Science. (f) Adsorption models of the $\mathrm{Zn}$ atom absorbed on CuZn $\mathrm{n}_{5}$ and $\mathrm{Zn}$. Reproduced with permission. ${ }^{132}$ Copyright 2019 , Wiley-VCH. (g) Zn deposition on the bare Zn electrode with or without a PA layer. (h) 2D diffusion of the Zn ion on the NE surface. (i) Side-view morphologies of Zn deposition behavior on Zn NEs with or without polyamide layer coating. Reproduced with permission. ${ }^{122}$ Copyright 2019 , The Royal Society of Chemistry. (j) Zn electrode morphology evolutions for the electrolyte with or without the diethyl ether additive. Reproduced with permission. ${ }^{133}$ Copyright 2019, Elsevier. 
surface, interrupting the normal charging/discharging processes and compromising the device performance. ${ }^{\mathbf{1 2 3}}$ It is worth mentioning that $\mathrm{Zn}$ dendrites would be intensified at large current densities for ZIHCs where high power densities are needed. ${ }^{\mathbf{1 2 4}}$ Moreover, the three types of problems are sometimes convoluted. For example, $\mathrm{Zn}$ dendrites will aggravate the corrosion and passivation of $\mathrm{Zn}$, because $\mathrm{Zn}$ dendrites have more low-coordination $\mathrm{Zn}$ atoms and defective lattice planes that can catalyze the corrosion process. ${ }^{\mathbf{1 2 5}}$ Therefore, for high-performance ZIHCs based on Zn metal NEs, addressing all these challenges is imperative and stringent.

In principle, the stable deposition of $\mathrm{Zn}$ ions is closely related to the nucleation and subsequent growth process. As shown by the free energy diagram in Fig. 14a, the initial nucleation of $\mathrm{Zn}$ needs to overcome a nucleation energy barrier to form a solid phase, after which the $\mathrm{Zn}$ further continues to be plated on the initially deposited layer. ${ }^{\mathbf{1 2 6 , 1 2 7}}$ The whole process can also be interpreted via the voltage profile (Fig. 14b), in which the nucleation overpotential is associated with the thermodynamic cost of forming a critical atom cluster (defined by the difference between the tip potential and the subsequent stable potential), while the plateau overpotential refers to that of zinc growth after initial nucleation. ${ }^{\mathbf{1 2 8}}$ Note that the solvation and desolvation of $\mathrm{Zn}$ ions are also a non-negligible factor for the $\mathrm{Zn}$ redox process. The electric double-layer structure (EDLS) in Fig. 14c illustrates the typical ion transport behavior at the $\mathrm{Zn}$ deposition interface. ${ }^{\mathbf{1 2 9}}$ Ions actuated by the electric field first move to the NE surface as a diffuse layer and then random nucleate at active sites, hence a smaller desolvation energy barrier of $\mathrm{Zn}^{2+}$ ions during this process is obviously more favorable. By analyzing all these factors relevant to $\mathrm{Zn}$ deposition, the design principles towards the homogenized interfacial electric field and ion flux can contribute to uniform ion nucleation and growth, thereby minimizing the unevenness of $\mathrm{Zn}$ growth.

5.1.1 Interfacial design between $\mathrm{Zn}$ and the substrate. $\mathrm{Zn}$ metal is a hostless NE that serves as both an active material and a current collector and thus is quite vulnerable due to the aforementioned problems. Therefore, a conductive substrate is necessary to maintain the shape integrity of the NEs, guide the growth of Zn metal, and more significantly, enable a high depth of discharge (DOD) of $\mathrm{Zn}$ species. So far, various conductive substrates such as carbon cloth, ${ }^{\mathbf{1 3 4}} \mathrm{CNT}$ paper, ${ }^{\mathbf{5 0}}$ graphene foam, ${ }^{135}$ MXene paper, ${ }^{136}$ nickel nanotubes, ${ }^{137}$ and copper foam $^{\mathbf{1 3 8}}$ have been used to host $\mathrm{Zn}$. Generally, these substrates possess a high active surface area, which can not only homogenize the electric fields but also provide enriched reactive sites to enable an even $\mathrm{Zn}$ stripping/plating. A typical example comes from $\mathrm{Lu}$ and co-authors, ${ }^{\mathbf{1 3 0}}$ who constructed 3D dendrite-free $\mathrm{Zn} / \mathrm{CNT}$ electrodes by depositing $\mathrm{Zn}$ on flexible 3D CNTs (Fig. 14d). The 3D CNT substrate endows the flexible symmetric cell with appreciably low voltage hysteresis $(27 \mathrm{mV})$ and decent cycling stability $(200 \mathrm{~h}$ ) with dendrite-free morphology at $2 \mathrm{~mA}$ $\mathrm{cm}^{-2}$, together with a DOD of $28 \%$.

Apart from the general substrate effects, Zn substrates can also be leveraged to guide the growth of $\mathrm{Zn}$. Zheng et al. ${ }^{131}$ reported a conceptually innovative substrate-guided epitaxy regulation strategy for $\mathrm{Zn}$ deposition. As shown in Fig. 14e, the intrinsic low thermodynamic free energy of exposed closest packed (0002) plane of $\mathrm{Zn}$ will lead to the random deposition of platelets. Instead, the aligned graphene, with a low lattice mismatch for Zn, can drive Zn to deposit with a locked crystallographic orientation. The resultant planar $\mathrm{Zn}$ electrode can be reversibly stripped and plated 10000 times with a high Coulombic efficiency of $99.9 \%$, even at $40 \mathrm{~mA} \mathrm{~cm}{ }^{-2}$. Note that the perfect carbon substrate is hydrophobic, which potentially hinders $\mathrm{Zn}$ deposition. Zhang et al. ${ }^{\mathbf{1 3 2}}$ argue that the binding energy between the zinc atom and carbon is weak, so that $\mathrm{Zn}^{2+}$ ions prefer to be plated on the initially nucleated $\mathrm{Zn}$ deposits rather than on the remaining carbon surface. Instead, they prove that copper shows intrinsic satisfactory zincophilicity, and $\mathrm{Zn}$ nucleation on copper surface is relatively uniform owing to the $\mathrm{Cu}-\mathrm{Zn}$ solid solution layer formed in situ during $\mathrm{Zn}$ plating (Fig. 14f). However, the metal substrate can substantially compromise the energy density as well as the flexibility of Zn NEs. In this context, carbon materials stand out because they offer good electrical conductivity, mechanical flexibility, and stability, yet with lightweight. Recently, Li's group ${ }^{139}$ demonstrated that surficial tin(Sn) modification bestows a 3D carbon felt host with higher zincophilicity, larger HER overpotential, and lower nucleation overpotential of $\mathrm{Zn}$ and thus induces uniform Zn plating/stripping in a better way with very a high Coulombic efficiency. Their work suggests that zincophilic hierarchical carbon materials can be a promising host for flexible $\mathrm{Zn}$ electrodes.

5.1.2 Interfacial design between $\mathrm{Zn}$ and the electrolyte. In essence, all electrochemical reactions occur at the $\mathrm{Zn}$-electrolyte interface, thus interfacial adjustments between $\mathrm{Zn} \mathrm{NE}$ and the electrolyte are pivotal to regulate the redox behaviors of $\mathrm{Zn}$ species. Unlike alkali metal electrodes (e.g., $\mathrm{Li}, \mathrm{Na}$ ), the solid electrolyte interphase (SEI) can hardly be formed on the $\mathrm{Zn}$ aqueous electrolyte interface; artificial SEI (A-SEI) layers are therefore developed as a very successful strategy to enhance the interfacial stability and cycling stability of $\mathrm{Zn}$ NEs. The core concept of A-SEIs is to protect the $\mathrm{Zn} \mathrm{NE}$ from directly coming in contact with electrolytes for regulating the nucleation distribution, ion transport, and resulting $\mathrm{Zn}$ stripping/plating behaviors. Due to the confined electrochemical environments on the $\mathrm{Zn}$-electrolyte interface by such A-SEI layers, dendritefree plating and side-reaction/passivation suppression can be achieved. In the last few years, various materials including polymers, ${ }^{\mathbf{1 4 0 - 1 4 2}}$ inorganic metal and metal oxides/salts, ${ }^{\mathbf{1 4 3 - 1 4 5}}$ carbon matrices, ${ }^{146-148}$ and metal-organic frameworks (MOFs) ${ }^{149}$ have been deployed to constitute efficient A-SEI layers on the $\mathrm{Zn}$ surface. A recent breakthrough was reported by Cui's group. ${ }^{\mathbf{1 2 2}}$ They systematically studied a polyamide (PA)-based A-SEI, which extrinsically suppressed the dendrite and side reactions plaguing bare Zn during electrochemical cycles. The physical PA layer served as a buffer layer that isolates active $\mathrm{Zn}$ from bulk electrolytes and also suppresses free water- $/ \mathrm{O}_{2}$-induced corrosion and passivation. Besides, the abundant amide groups within the PA interphase layer can coordinate with $\mathrm{Zn}^{2+}$ ions to positively refine the nucleation grains by elevating the nucleation overpotential and increase the nucleation density via 
restricting $\mathrm{Zn}^{2+} 2 \mathrm{D}$ diffusion, leading to a dense and smooth $\mathrm{Zn}$ deposition (Fig. 14g-i). As expected, the PA modified Zn NE sustains reversible, dendrite-free plating/stripping for over 8000 hours and a high areal capacity of $10 \mathrm{~mA} \mathrm{~h} \mathrm{~cm}^{-2}$ with an exceptional DOD of $85 \%$. Note that the A-SEI layers are not necessarily extrinsically coated. They can be generated in situ via elaborated surficial transformations. For example, Hao et al. ${ }^{\mathbf{1 5 0}}$ built a compact artificial ZnS layer on the Zn metal surface by an in situ vapor-solid strategy. The optimized ZnS layer not only suppressed $\mathrm{Zn}$ corrosion by forming a physical barrier on the $\mathrm{Zn}$ surface but also inhibited dendrite growth via guiding the $\mathrm{Zn}$ plating/stripping underneath the artificial layer. Similarly, Yang

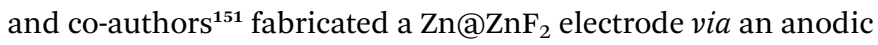
growth method, in which the $\mathrm{ZnF}_{2}$ matrix served as an A-SEI layer guiding the even deposition of $\mathrm{Zn}$ underneath.

In addition to A-SEI layer coating on the $\mathrm{Zn}$ surface, adjusting from the electrolyte side is a complementary method to address $\mathrm{Zn}$ interfacial issues. In this regard, adding additives to electrolytes to adjust $\mathrm{Zn}^{2+}$ ion transport and surface current distribution is one common method. For example, Xu et al. ${ }^{133}$ found that when a small amount ( 2 vol\%) of diethyl ether was introduced into the electrolyte, the highly polarized ether molecules will preferentially adsorb onto the protrusions of $\mathrm{Zn}$ due to the high local electrical field. The deposition of $\mathrm{Zn}^{2+}$ ions is then extruded to the flat areas, thereby diminishing the initial morphological fluctuations in long-term cycles. In another work, $\mathrm{Na}_{2} \mathrm{SO}_{4}$ has been reported to alleviate the formation of $\mathrm{Zn}$ dendrites based on the electrostatic shielding mechanism of $\mathrm{Na}^{+}$ions. ${ }^{153}$ Besides, the additive molecules can also manipulate the $\mathrm{Zn}$-electrolyte surface via tuning the solvation sheath of $\mathrm{Zn}^{2+}$ ions. A recent study suggested that ethylene glycerol can substitute water molecules in the $\mathrm{Zn}^{2+}$ ion solvation sheath to increase its nucleation overpotential and circumvents parasitic reactions during cycling. ${ }^{154}$ Of note, suitable adhesion of organic additives can improve the durability and prevent the premature failure of the $\mathrm{Zn}$ electrode, but inordinate adhesion may disturb the diffusion of electrolyte ions and the intrinsic conductivity of the electrode. ${ }^{155}$ It should also be mentioned that, due to specific interactions between the functional groups and the $\mathrm{Zn}$ surface, some polyelectrolytes have recently been reported to render stable $\mathrm{Zn}$-electrolyte interfaces during the charging/discharging process. ${ }^{156,157}$ These intrinsic properties of polyelectrolytes can be potentially leveraged in flexible ZIHCs.

\subsection{Zn metal-free NEs}

Despite many efforts that have been devoted to addressing the problems associated with Zn NEs, the utilization of Zn NEs remains problematic. Enlightened by the "rocking-chair"-type lithium-ion batteries, deploying Zn metal-free NEs that can store $\mathrm{Zn}^{2+}$ ions with an adsorption or intercalation mechanism (Fig. 15a and b) at low potentials will fundamentally circumvent the aforementioned problems. Zinc metal-free NEs should
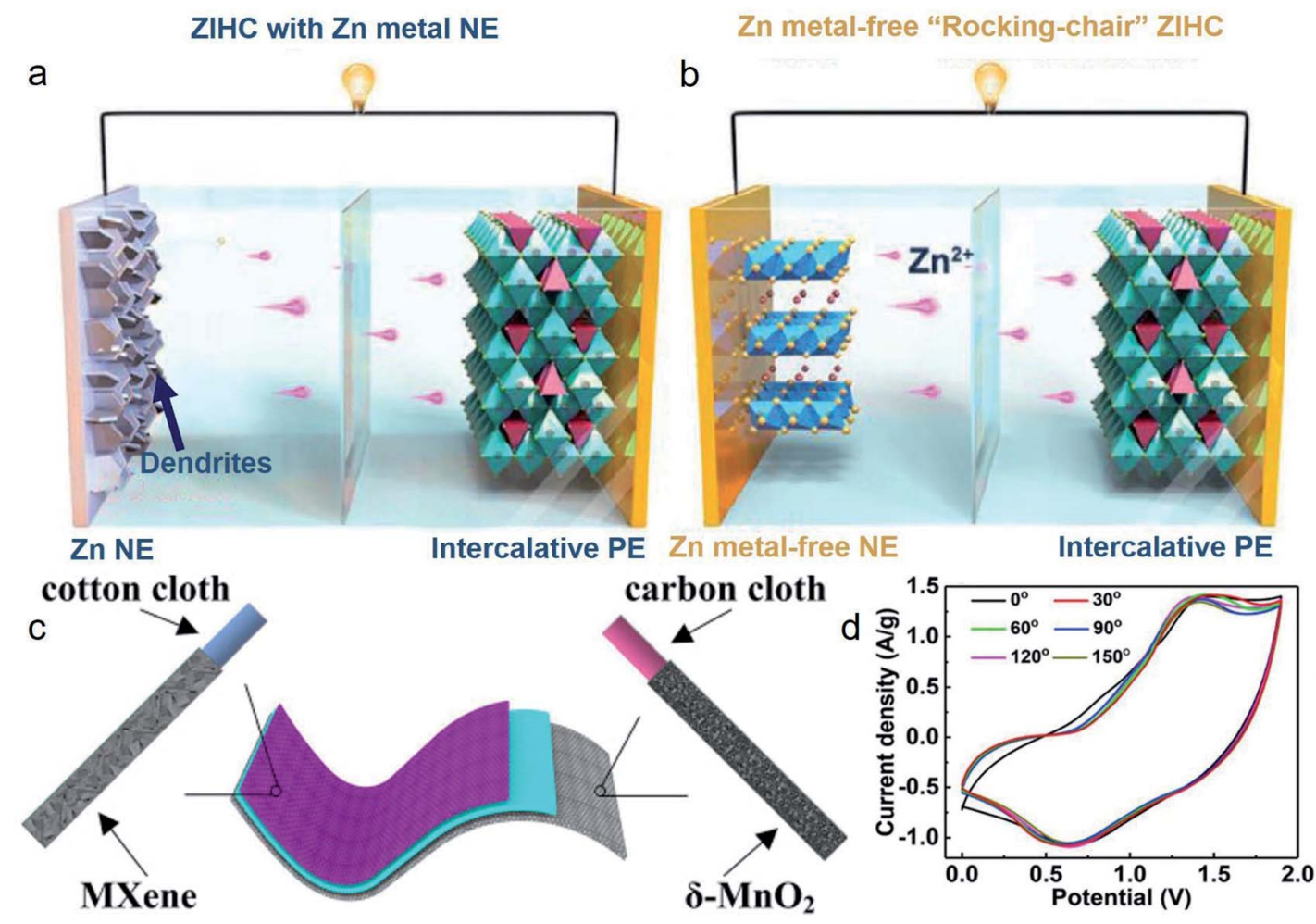

Fig. 15 (a) ZIHCs with Zn metal NE and intercalative PE and (b) Zn metal-free "rocking-chair" type ZIHCs with intercalative PE and Zn metal-free NE. Reproduced with permission. ${ }^{152}$ Copyright 2020, Wiley-VCH. (c) Schematic diagram of the flexible $\mathrm{ZIHC}_{\mathrm{Consists}}$ of $\delta$-MnO $\mathrm{MCC}_{2}$ and MXeneaCOC electrodes. (d) CV curves at $10 \mathrm{mV} \mathrm{s}^{-1}$ when the ZIHC was bent to different angles. Reproduced with permission. ${ }^{94} \mathrm{Copyright} 2019$, Elsevier. 
possess: (1) appropriate potential versus $\mathrm{Zn} / \mathrm{Zn}^{2+}$, (2) excellent structural stability without dissolution issues, (3) high initial Coulombic efficiency, (4) prompt and reversible intercalation/ deintercalation dynamics. ${ }^{158}$ Recently, the MXene materials have been studied as potential Zn metal-free NEs due to their excellent metal conductivity, hydrophilic surface, and intercalation/de-intercalation energy storage mechanism. As mentioned above, Wang et al. ${ }^{67}$ applied a free-standing MXene $\left(\mathrm{Ti}_{3} \mathrm{C}_{2} \mathrm{~T}_{x}\right)$ film as a capacitor-type $\mathrm{NE}$, which results in a stable electrochemical performance with a capacitance retention of 83.6\% after 15000 cycles and a Coulombic efficiency of above 93.3\% during the cycles. Moreover, the as-fabricated ZIHCs exhibited excellent flexibility that can maintain around $94.6 \%$ capacitance even when bent up to $120^{\circ}$. Subsequently, Zeng and co-workers ${ }^{94}$ fabricated a flexible NE by anchoring the MXene $\left(\mathrm{Ti}_{3} \mathrm{C}_{2} \mathrm{~T}_{x}\right)$ nanosheet onto a cotton cloth (COC) substrate. Their assembled ZIHC (Fig. 15c) not only possesses good cycling stability ( $\sim 80.7 \%$ capacitance retention after 16000 cycles) but also exhibits decent flexibility (93\% capacitance retention even when the ZIHCs were bent up to $150^{\circ}$, see Fig. 15d). Nevertheless, though the $\mathrm{Zn}$ metal-free electrodes guarantee a safer working mechanism with improved cycling lifespan, all their electrochemical performances (both gravimetric and volumetric capacity and energy density) are far from satisfactory for practical applications.

\section{Considerations for flexible devices}

\subsection{Flexibility and softness}

The overall construction of flexible devices can be customized to cater to various on-demand applications. Currently, onedimensional fibrous, 2D planar, and 3D stereostructured ZIHCs have been developed (Fig. 16a-c).. ${ }^{41,93}$ Although increasingly reported, most works are performance-oriented without a unified standard to describe the "flexible issues" of the flexible devices. Zhi's group ${ }^{159}$ proposed a standard protocol for describing flexibility based on bending tolerance tests. As shown in Fig. 16d, the device in the bending state can be described by three independent parameters, namely, the length of the device $(L)$, the angle of bending $(\theta)$, and the bending radius $(R)$. A change in any one of these parameters can dramatically differ in the device's force area. For instance, the stress area changes significantly with the change in the bending angle $(\theta)$ when keeping the device length $(L)$ and bending radius $(R)$ constant (Fig. 16d). Furthermore, it was found that all three parameters have an impact on the electrochemical performance of the energy storage devices.

To depict a full vision of a given flexible energy storage device, the authors also suggest reporting its "softness", a parameter defined according to the international standard method for evaluating the softness of leather (ISO 17235). In detail, a cylindrical rod with a certain mass is lowered at a certain rate onto a fixed flexible material, and the resulting change in the flexible material is recorded as the softness (typically in $\mathrm{mm}$ ). The softness is closely related to three factors: (1) the material of the flexible current collector; (2) the electrode type; and (3) the thickness of the active material. For some devices aiming to obtain a high energy density, the increase of the active material will significantly diminish the device's softness. Therefore, these four key parameters afford valuable guidelines that should be clarified in future flexible energy storage devices for better cross-lab comparison.

\subsection{Multifunctionality}

6.2.1 Temperature adaptability. As mentioned above, aqueous ZIHCs are more promising for their higher rate
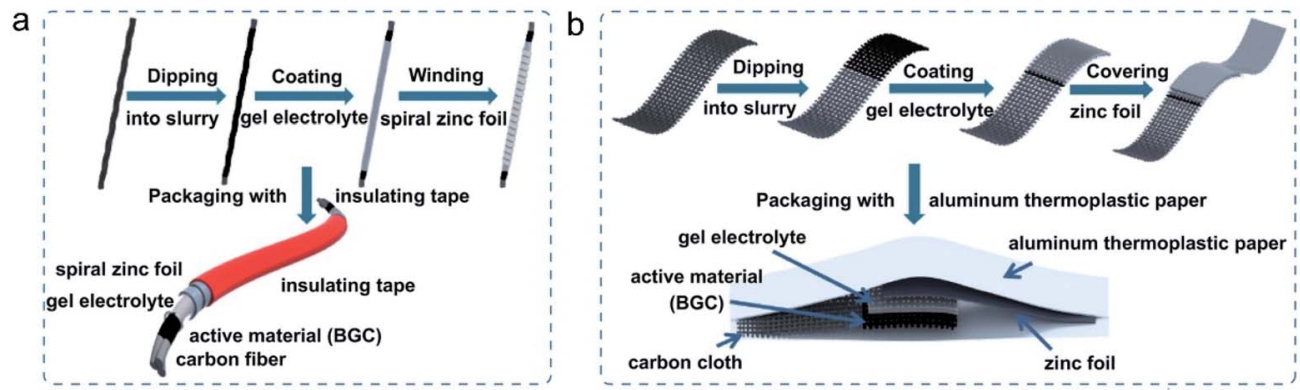

C
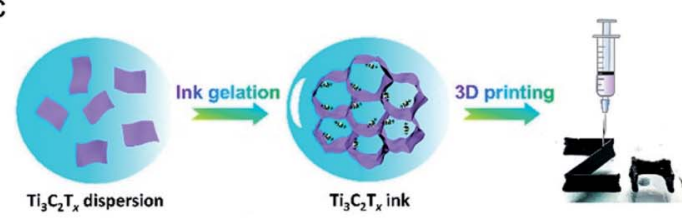

d

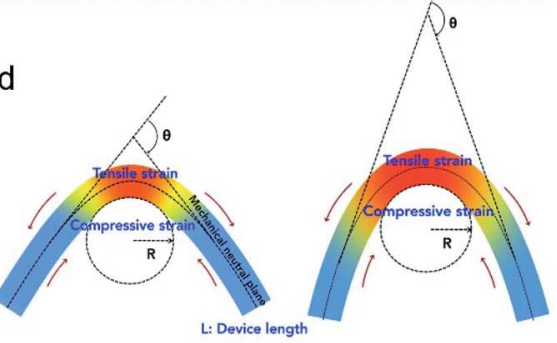

Fig. 16 Schematic diagram of ZIHCs with different electrode designs: (a) fibrous. (b) Planar, Reproduced with permission. ${ }^{41}$ Copyright 2021 , Springer Nature. (c) Stereo using 3D printing. Reproduced with permission. ${ }^{93}$ Copyright 2021, American Chemical Society. (d) Schematic diagram of the structure of a flexible device and its three key parameters $\left(L, \theta\right.$ and $R$ ). Reproduced with permission. ${ }^{159}$ Copyright 2019 , Elsevier. 
capability and intrinsic safety. However, the aqueous GPEs in ZIHCs are susceptible to temperature changes, since sub-zero temperatures will incur freezing and high temperatures may lead to dry-out of the electrolyte, both resulting in degradation or even failure of the devices. The highest recorded temperature on Earth is $58{ }^{\circ} \mathrm{C}$, and the lowest one is $-89.2{ }^{\circ} \mathrm{C} .{ }^{160}$ Hence, the temperature-adaptability of flexible ZIHCs is a vital requirement. Recently, flexible ZIHCs with freezing resistance have been reported by introducing anti-freeze hydrogel electrolytes. ${ }^{57,58,161}$ For example, Li and colleagues ${ }^{57}$ fabricated a PVAbased flexible electrolyte by dispersing PVA in a mixture of ethylene glycol (EG), $\mathrm{H}_{2} \mathrm{O}$, and $\mathrm{Zn}\left(\mathrm{CF}_{3} \mathrm{SO}_{3}\right)_{2}$, followed by a freeze-thawing treatment. The EG was introduced to break the intermolecular hydrogen bonding, hence lowering the freezing point of the electrolyte. The as-synthesized hydrogel electrolyte possesses decent freezing resistance and flexibility and maintains a decent conductivity $\left(4.02 \mathrm{mS} \mathrm{cm}^{-1}\right)$ after seven days at $-15{ }^{\circ} \mathrm{C}$.

Aside from the organic cryoprotectants, the intrinsic colligative property of some inorganic salts can be leveraged for the temperature-adaptability. Wang et al. ${ }^{58}$ reported a $7.5 \mathrm{~m} \mathrm{ZnCl}_{2}$ - filled PAM GPE, which possesses an ideal freeze resistance with flexibility and good ionic conductivity $\left(17.9 \mathrm{mS} \mathrm{cm}^{-1}\right)$ even at $-20{ }^{\circ} \mathrm{C}$ (Fig. 17a). The flexible ZIHCs assembled from this GPE exhibited a high capacity retention of $70 \%$ at $1 \mathrm{~A} \mathrm{~g}^{-1}$ when the temperature drops from 25 to $-20{ }^{\circ} \mathrm{C}$ (Fig. 17b). A cellulosebased hydrogel containing $\mathrm{ZnCl}_{2}$ and $\mathrm{CaCl}_{2}$ has also been used for ZIHCs working at low temperatures. ${ }^{161}$ Recently, Zhang and co-authors ${ }^{162}$ uncovered that the $7.5 \mathrm{~m} \mathrm{ZnCl}_{2}$ solution can bring a solid-liquid transition temperature down to $-114{ }^{\circ} \mathrm{C}$ (Fig. 17c). After the addition of $\mathrm{ZnCl}_{2}$, the resulting $\mathrm{Cl}^{-}$-containing $\mathrm{Zn}^{2+}$ clusters interact more strongly with $\mathrm{H}_{2} \mathrm{O}$, thus enhancing the ionic interactions and breaking the hydrogen bonding network, which eventually leads to the low freezing point. This work suggests that flexible ZIHCs can work at extremely low temperatures by deploying suitable $\mathrm{Zn}$ salts. In addition to the freeze resistance at low temperatures, the devices' thermal stability/safety at high temperatures is another important consideration. In this regard, Yang et al. ${ }^{\mathbf{1 6 3}}$ recently demonstrated a smart and thermally recoverable flexible devices by using a $\mathrm{ZnCl}_{2}$-based PAM GPE, as illustrated in Fig. 17d. At elevated temperatures, the water evaporation
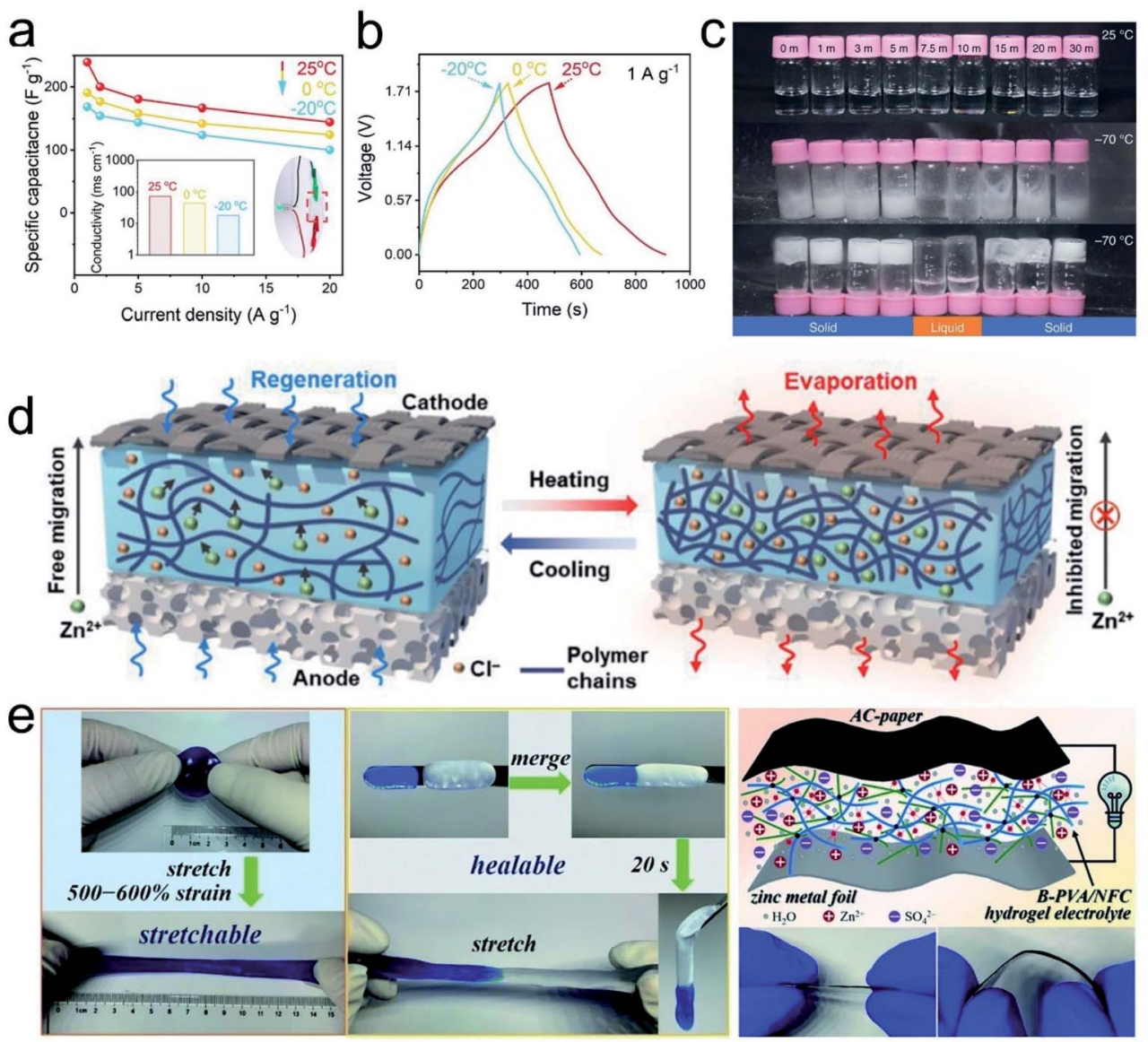

Fig. 17 (a) Rate capability of $\mathrm{ZIHC}$ with $7.5 \mathrm{~m} \mathrm{ZnCl}_{2}$ at different temperatures; the inset shows the ionic conductivity of PAM (7.5 $\mathrm{m} \mathrm{ZnCl}$ ) and the photo demonstrates that an LED was lighted up through PAM that served as an ionic conductor. (b) GCD curves at different temperatures. Reproduced with permission. ${ }^{58}$ Copyright 2021 , Wiley-VCH. (c) Images of different concentrations of $\mathrm{ZnCl}_{2}$ at $25^{\circ} \mathrm{C}$ and $-70{ }^{\circ} \mathrm{C}$. Reproduced with permission. ${ }^{162}$ Copyright 2020, Springer Nature. (d) Principle of thermal self-protection of hygroscopic $\left(\mathrm{ZnCl}_{2}\right)$ gel electrolytes. Reproduced with permission. ${ }^{163}$ Copyright 2020, Wiley- $\mathrm{VCH}$. (e) Stretching demonstration of the flexible GPE and the schematic illustration of the flexible ZIHC. Reproduced with permission. ${ }^{88}$ Copyright 2019, The Royal Society of Chemistry. 
decreases the ionic diffusion of the GPE to avoid thermorunaway of the device. The electrolyte with hygroscopic properties (from $\mathrm{ZnCl}_{2}$ ) can then absorb water from the air to restore its original function when it returns to a low temperature.

6.2.2 Self-healable devices. For flexible energy storage devices, repeated deformations and/or strong external forces may cause a fracture in GPEs and/or displacement of electrodes, hence self-healable devices are highly preferred. Important features to achieve self-healing properties are the presence of chemical bonding or hydrogen bonding, which are usually reversibly cross-linked within GPEs. For example, the $\mathrm{B}(\mathrm{OH})_{4}$ moiety in borax can dynamically cross-link with hydroxyl groups widely present in the gel backbones. The formed B-O chemical bonds can be easily and rapidly reconstructed after a fracture. Thus, borax is widely used as a cross-linker for self-healable GPEs. Based on this, Chen et al. ${ }^{\mathbf{8 8}}$ used borax to cross-link PVA and nano-fibrillated cellulose together with $\mathrm{ZnSO}_{4}$ to synthesize a GPE, which exhibited $98 \%$ self-healed mechanical properties only after $20 \mathrm{~s}$ (about $600 \%$ strain and $22.5 \mathrm{kPa}$ ), as shown in Fig. 17e. In another work, Ni et al. ${ }^{\mathbf{1 0 8}}$ constructed a selfhealable GPE by utilizing hydrogen bonding in PVA. However, the force of hydrogen bonding is relatively weaker as compared with chemical bonding. It is worth mentioning that the selfhealable devices are very preliminary, since in most reported sandwich-structured ZIHCs, the self-healing functionality is based on the GPEs. The all-in-one type flexible devices that incorporate all other components (e.g., electrode, current collector) with the GPEs can be a potential candidate for realizing the self-healing function at the whole device level.

6.2.3 Anti-self-discharging capability. The instability of physical charge adsorption leads to severe self-discharging in ZIHCs. Therefore, for flexible ZIHCs, self-discharging resistance is also an important part of their versatility. As exemplified in the case of $\mathrm{TiN}-\mathrm{SO}_{4}{ }^{2-},{ }^{91}$ screening of suitable electrolyte ionelectrode material pairs that have more tight and stable adsorption is an ideal way to achieve the function of anti-selfdischarging. Besides, such a function can be achieved by using insertion-type and conversion-type electrode materials (e.g., phosphorene), which can better confine ions. ${ }^{80}$ Also, a proper GPE design can also enable self-discharging resistance. For example, Liu et al. ${ }^{164}$ proposed that a unique zwitterionic gel of poly-propylsulfonate dimethylammonium
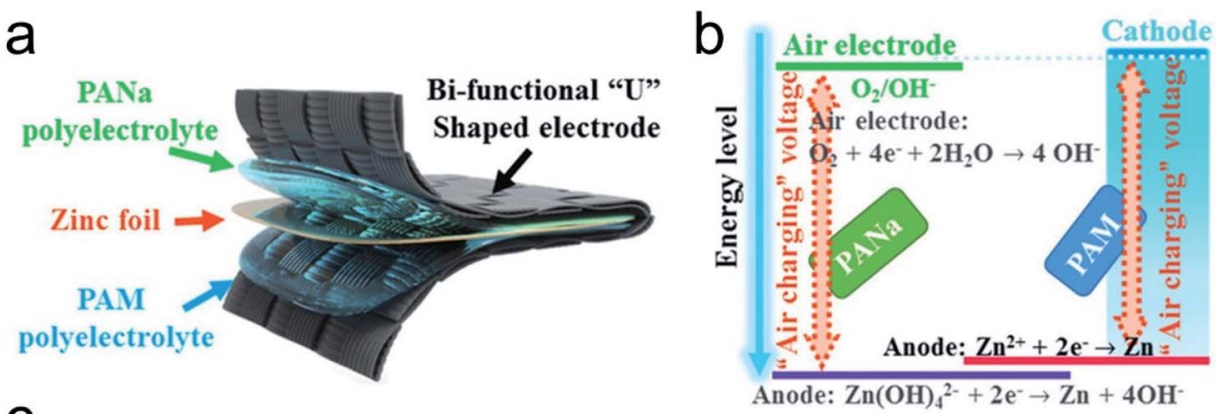

C
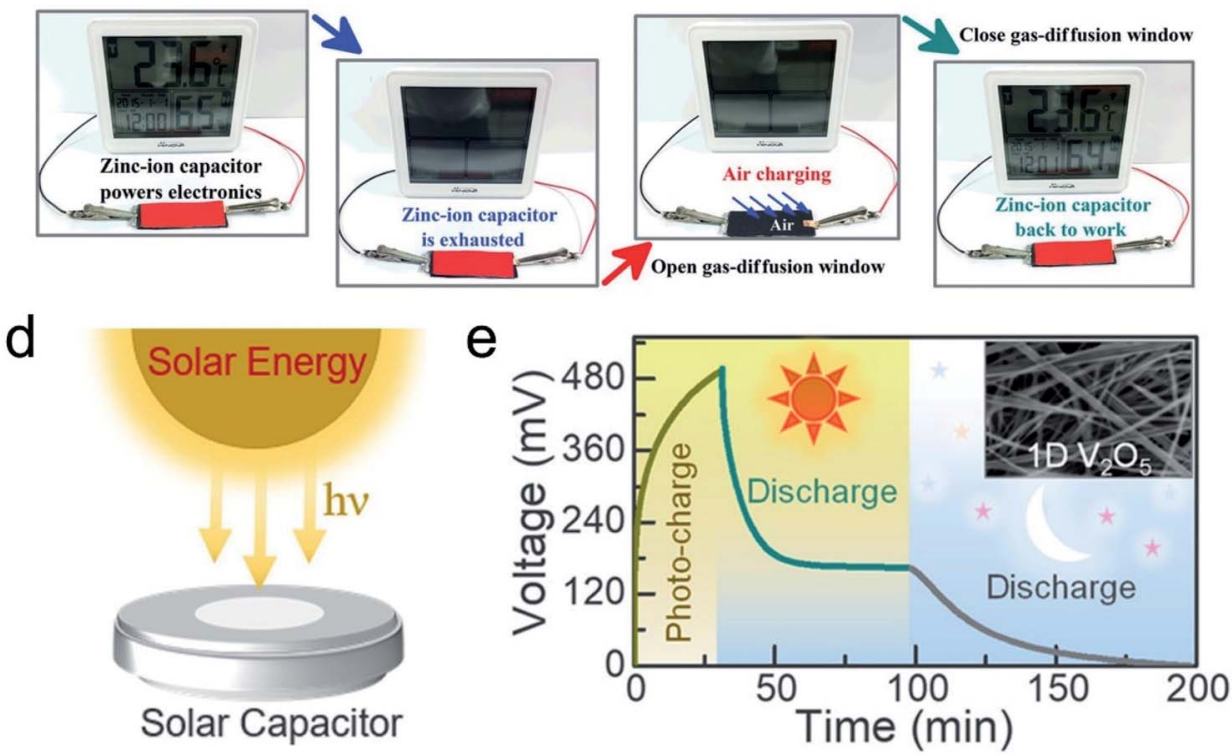

Fig. 18 (a) Schematic of a U-shaped air self-chargeable hybrid system. (b) Working principles of the air self-chargeable hybrid system. (c) The demonstration of powering a device. Reproduced with permission. ${ }^{165}$ Copyright 2019, Wiley-VCH. (d) Schematic of the photo-rechargeable ZIHCs. Reproduced with permission. ${ }^{166}$ Copyright 2018, American Chemical Society. (e) Photocharge and discharge in the illuminated condition of the photo-rechargeable $\mathrm{ZIHC}$ using $1 \mathrm{D} \mathrm{V} \mathrm{O}_{5}$. Reproduced with permission. ${ }^{167}$ Copyright 2018, American Chemical Society. 
propylmethacrylamide (PPDP) can inhibit the ion diffusion on the electrode by the unique electrostatic attraction between anions and cations present in the gel structure. Specifically, they proposed that by applying an external electric field, channels allowing ions to move rapidly will form due to the orientation of the polyzwitterionic chains within the PPDP GPE. When the device is left in an open-circuit condition, these channels would close because of the more random orientation of the polyzwitterionic chains and thus is unfavorable for the diffusion of the ions and redox species resulting from the increased steric barriers. This additional advantage of anti-selfcharging suggests the great potential of GPEs, which definitely deserves further investigation.

\subsection{Self-chargeable hybrid system}

For energy storage devices, achieving a higher energy density has been a constant pursuit for a longer lifetime. Integrating energy storage devices with energy conversion units can be an effective way to extend the capacity of energy storage devices. This integrated/hybrid system can harvest and replenish energy from the environment when energy is exhausted in ZIHCs. Moreover, the hybrid system can avoid extra external electronics to adjust and match the voltage/current output, thereby reducing the interfacial charge transfer resistance and separations among different components. Thus, combining ZIHCs with other energy conversion systems allows for a more promising self-charging system. For example, Ma and colleagues ${ }^{\mathbf{1 6 5}}$ demonstrated an air-chargeable flexible ZIHC by employing a unique U-shaped electrode sandwiching a zinc electrode and two different polymer electrolytes, as shown in Fig. 18a. In this configuration, the zinc electrode can support both systems.

The GPE constructed by PAM and $\mathrm{ZnSO}_{4}$ mainly builds a ZIHC, while another GPE consisting of sodium polyacrylate (PANa) and $\mathrm{KOH}$ builds a $\mathrm{Zn}$-air cell to convert $\mathrm{O}_{2}$ to energy
(Fig. 18b). This flexible hybrid system achieved rapid charging of depleted ZIHCs to $88 \%$ in $10 \mathrm{~min}$ and good stability during 60 venting-charging cycles, which was able to power an electronic device through air charging, as demonstrated in Fig. 18c. However, the $\mathrm{Zn}$-air unit is an open system, and the alkaline GPE will react with $\mathrm{CO}_{2}$ in air, generate by-products, and corrode the zinc electrode. Therefore, it is an effective solution to build a joint system by using a safer neutral solution. Bearing this in mind, Sun et al. ${ }^{168}$ reported another advanced design that applied mild electrolytes $\left(\mathrm{ZnSO}_{4}\right.$ and $\left.\mathrm{Zn}(\mathrm{Ac})_{2}\right)$ to the entire cell, building a safer and more stable hybrid system.

Along with air, sunlight is one of the most common and widely used green energy sources. Photo self-charging is another novel self-chargeable hybrid system design strategy. The proof-of-concept study (Fig. 18d) was conducted by Boruah et al. and was demonstrated by using $2 \mathrm{D}$ graphitic carbon nitride ${ }^{\mathbf{1 6 6}}$ and $\mathrm{V}_{2} \mathrm{O}_{5}$ semiconductors ${ }^{\mathbf{1 6 7}}$ (Fig. 18e), respectively. Without any additional photovoltaic or electrical devices, a bare self-charging hybrid system driven by solar energy is achieved. In short, air self-charging and photo self-charging provide inspirations for building other self-chargeable hybrid systems by combining ZIHCs with other energy conversion units, such as triboelectric nanogenerators.

\subsection{Miniaturization}

Miniaturization is a major trend in the development of portable and highly integrated electronics, which is the key to the realization of "artificial intelligence" and "Internet of things" in the future. , $50,170-172$ However, with the existing miniaturized ESSs, it is difficult to achieve high energy and power density simultaneously, which hinders further miniaturization of many electronic products. ZmSCs have better electrochemical properties due to miniaturization and reduced electron transfer distance, showing promise to bridge the demand gap. ${ }^{89}$ Also, it should be

a

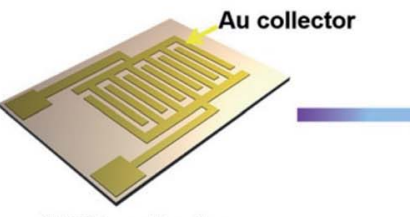

(i) Ti/Au patterning

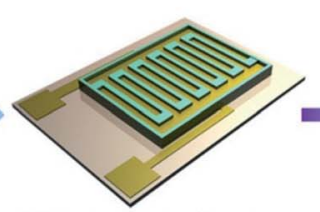

(ii) Photoresist patterning

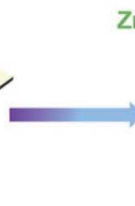

Zn nanosheet

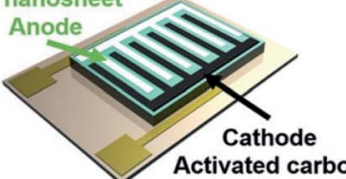

(iii) Active materials electrodeposition and injection
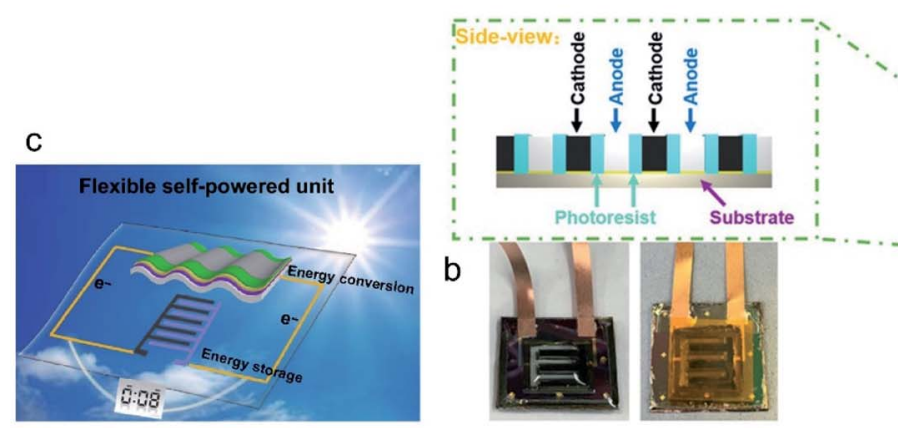$$
\text { electrits }
$$

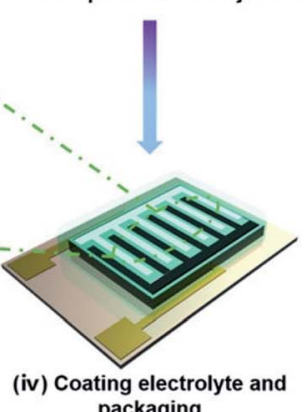

packaging

Fig. 19 (a) Fabrication process of ZmSC. (b) Images of ZmSC. Reproduced with permission. ${ }^{89}$ Copyright 2019, Wiley-VCH (c) Diagram of the integrated flexible self-powered ZIHC combining ZmSC and miniaturized solar cells. Reproduced with permission. ${ }^{169}$ Copyright 2020 , Springer Nature. 
noted that the evaluation of electrochemical performances of ZmSCs is different from those of other ZIHCs. The areal or volumetric density is more critical than the gravimetric energy density, since the weight of active materials is negligible in miniaturized ESSs. ${ }^{173}$ Sun et al. ${ }^{50}$ fabricated miniaturized CNTbased electrodes by laser carving and constructed the first flexible $\mathrm{ZmSC}$, which delivered a decent areal capacitance of $83.2 \mathrm{mF} \mathrm{cm}^{-2}$ at $1 \mathrm{~mA} \mathrm{~cm}{ }^{-2}$. Although this constructed $\mathrm{ZmSC}$ was yet to be improved in terms of electrochemical performance and cyclability, it represents an important reference for miniaturization in this field. Following this, Zhang et al. ${ }^{89}$ optimized the electrode material (AC as $\mathrm{PE}$ ), collector ( $\mathrm{Au}$ foil), and fabrication process of ZmSC (Fig. 19a and b). An ultra-high capacitance of $600 \mathrm{mF} \mathrm{cm} \mathrm{cm}^{-2}$ at the current density of $3.12 \mathrm{~mA} \mathrm{~cm}^{-2}$ was achieved in their devices. Apart from performance advancement, Zeng et $a l .{ }^{\mathbf{1 6 9}}$ argued that the lack of a matching miniaturized charging system to the miniaturized ZIHCs remains a huge challenge. They constructed a flexible selfpowered ZIHC by combining a ZmSC fabricated from an asymmetric printing technology with a miniaturized solar cell (Fig. 19c). This miniaturized device features a fast photovoltaic conversion character that can charge the energy storage unit to $1.6 \mathrm{~V}$ in $23 \mathrm{~s}$. Their work put forward a potential consideration direction for micro-ZIHCs.

\section{Conclusions and outlooks}

In summary, by integrating the complementary advantages of both Zn-ion batteries-for high energy density-and conventional SCs - for exceptional power density and cycling stability, flexible ZIHCs depict promising prospects as a reliable power source for realizing the full vision of emerging flexible electronics. In this review, we outlined the fundamental principles, recent progress, and critical challenges of flexible ZIHCs. Key emphasis was given toward general design principles and specific considerations of electrode materials, electrolytes, electrode-electrolyte interfacial properties, and functional device innovations in the backdrop of flexible ZIHCs. In general, despite the thriving and prompt development since their emergence a few years ago, the application of flexible ZIHCs is still in its initial stage. Here, we propose a few special considerations which we believe should be the research focus in the near term:

\subsection{Improving the SCD of $3 \mathrm{D}$ carbon materials}

Carbon materials are the most promising materials for ZIHCs because of their incomparable advantage of capacitive behaviors. In this regard, PCs with a large SAA and hierarchical porosity can enhance both the capacity and rate capability. Conventional template methods combined with suitable activation treatments can hopefully achieve such on-demand structural tunings. Heteroatom doping can bring in additional adsorption sites in PC and potentially alleviate the selfdischarging due to the relatively stable chemical bonds compared with pure physical adsorption, but the mechanism needs further in-depth clarification. Especially, the interactions between the $\mathrm{Zn}^{2+}$ ions and the dopant sites (along with possible defect sites) and the interfacial adsorption/desorption kinetics call for the collaboration of experimental tests, theoretical calculations, and simulation characterization. For flexible devices, interfacial contact is a significant concern, while the volumetric energy density is a more relevant and significant metric. Therefore, 3D carbon materials with a better interfacial contact and higher SCDs will be ideal electrode materials for flexible ZIHCs.

\subsection{Developing advanced pseudocapacitive materials}

Currently, TMOs and their derivatives (e.g., layered-doublehydroxides), conductive polymers, MXenes, MOF materials, and delaminated phosphorene provide more opportunities for the development of ZIHCs. However, they all suffer from limited cycling stability due to poor conductivity and/or chemical/ structural instability. Future endeavors using strategies such as substrate coupling, doping, pre-intercalation, and nano-array structuring can hopefully enhance the electrical conductivity, ion diffusion kinetics, and electrochemical stability of these pseudocapacitive materials. Meanwhile, equal efforts should be given to developing novel pseudocapacitive materials. To this end, we envisage that lamellar 2D pseudocapacitive materials are particularly promising because they offer a balance between capacity, diffusion kinetics, and anti-self-discharging property.

\subsection{Designing compatible flexible current collectors}

Most of the developed materials exist in the powder form, hence extra binders are indispensable to cast them onto certain current collectors. As mentioned above, the typical metal current collectors (e.g., copper, silver) may become problematic in ZIHCs because of the galvanic corrosion toward Zn metal (by forming copper-zinc or silver-zinc primary cells). Also, considering the repeated deformations (e.g., bending, stretching, and twisting) in usage scenarios of flexible ZIHCs, metal current collectors are very vulnerable. In this context, carbonbased electrodes (e.g., 3D carbon fibers) stand out since they concurrently offer satisfying electrical conductivity, mechanical flexibility, and stability, yet with lightweight. However, integrating active materials onto flexible carbon substrates requires further study.

\subsection{Engineering electrolytes for flexible ZIHCs}

For fundamental studies in liquid electrolytes, special considerations should be given to the solvation structure and associated desolvation energy of the solvated $\mathrm{Zn}^{2+}$ ion clusters. The influence of different anions is non-negligible, and the choice of anions is largely influenced by the electrode materials. Therefore, in addition to typical requirements such as the high ionic conductivity, low viscosity, and wide voltage window, overall considerations for the impact on both PE and NE are mandatory. For flexible ZIHCs, the GPE is a salient component not only to conduct charges between $\mathrm{PE}$ and $\mathrm{NE}$ but also to serve as a separator to provide ionic movement channels. Besides, the electrode-electrolyte interface properties need careful consideration. In this backdrop, with the basic requirements of ionic 
conductivity and mechanical properties, further efforts should be reasonably devoted to enhancing the interfacial contact and understanding the interfacial properties. We propose that hierarchically porous and cross-linked GPEs with a fibril surface can enhance the interfacial contact and maintain the structural stability in flexible ZIHCs. Furthermore, coating electrodes with in situ synthesized GPE is a promising strategy to boost the interfacial contact. Moreover, future research studies can focus on the GPE-electrode interface to intrinsically address the notorious dendrite growth, metal dissolution, and selfdischarging problems.

\subsection{Boosting the cycling stability of Zn NEs}

In light of their intrinsic high volumetric energy density, nanostructured $\mathrm{Zn}$ metal-based NEs can be a promising candidate for flexible ZIHCs. To achieve this, the dualinterfacial design deserves particular research efforts. As justified in Section 5, zincophilic hierarchical carbon materials can be a promising host for flexible $\mathrm{Zn}$ electrodes, which not only guarantees high conductivity and flexibility but also guides the growth of $\mathrm{Zn}$ metal. For the $\mathrm{Zn}$-electrolyte interface, coating with a suitable A-SEI layer is reliable but stills need further investigations. Advanced protocols in other metal-electrode (e.g., $\mathrm{Li}, \mathrm{Mg}$ ) batteries can be extended for $\mathrm{Zn}$ NEs. We propose that the concurrent consideration of the A-SEI layer and the GPE materials may simplify future explorations.

\subsection{Improving the volumetric metrics on the whole device level}

One crucial challenge of flexible electronics is to design energy storage devices that are flexible, light, and thin. Hence, high volumetric metrics are essential to flexible ZIHCs. In most existing studies, the use of low-density active materials (e.g., carbon) and excessive $\mathrm{Zn}$ foil/plate will greatly diminish the volumetric metrics on the whole device level. Further studies should be devoted to matching the NE/PE weight ratios, minimizing the electrolyte content, achieving compact configurations, and the development of energy-dense electrode materials. Critical information such as the DOD value of $\mathrm{Zn} \mathrm{NE}$ and the geometric structure of the whole cell should be provided to better advance the practical applications of ZIHCs.

\subsection{Standardizing flexible tests}

Most reported works usually adopt simple bending, twisting, and stretching tests by hand to evaluate the mechanical flexibility and durability, without providing quantitative data pertinent to the modulus, tensile strength, etc., which seems somewhat arbitrary. ${ }^{159}$ To fulfill the eventual expectation of flexible vision, it is imperative to establish systematic criteria to evaluate the mechanical flexibility and durability of flexible ZIHCs.

\section{Author contributions}

C. W., writing - original draft; X. Z., writing - original draft; P. J. C., writing - review \& editing; Z. P., conceptualization, writing review \& editing, and project management.

\section{Conflicts of interest}

There are no conflicts to declare.

\section{Acknowledgements}

The authors thank the financial support from the Australian Research Council Discovery Early Career Researcher Award (DE200101669). X. Z. thanks the support from the China Scholarship Council (CSC 202008200011), the Ministry of Education of China.

\section{Notes and references}

1 D. P. Dubal, N. R. Chodankar, D. H. Kim and P. GomezRomero, Chem. Soc. Rev., 2018, 47, 2065-2129.

2 T. Lv, M. Liu, D. Zhu, L. Gan and T. Chen, Adv. Mater., 2018, 30, e1705489.

3 J. Shi, S. Liu, L. Zhang, B. Yang, L. Shu, Y. Yang, M. Ren, Y. Wang, J. Chen, W. Chen, Y. Chai and X. Tao, Adv. Mater., 2020, 32, e1901958.

4 S. Zhai, H. E. Karahan, C. Wang, Z. Pei, L. Wei and Y. Chen, Adv. Mater., 2020, 32, e1902387.

5 M. R. Lukatskaya, B. Dunn and Y. Gogotsi, Nat. Commun., 2016, 7, 12647.

6 Y. Shao, M. F. El-Kady, J. Sun, Y. Li, Q. Zhang, M. Zhu, H. Wang, B. Dunn and R. B. Kaner, Chem. Rev., 2018, 118, 9233-9280.

7 D. P. Dubal, O. Ayyad, V. Ruiz and P. Gomez-Romero, Chem. Soc. Rev., 2015, 44, 1777-1790.

8 P. Simon and Y. Gogotsi, Nat. Mater., 2008, 7, 845-854.

9 G. Amatucci, F. Badway and A. Du Pasquier, J. Electrochem. Soc., 2000, 99, 344-359.

10 V. Aravindan, J. Gnanaraj, Y. S. Lee and S. Madhavi, Chem. Rev., 2014, 114, 11619-11635.

11 J. Ding, H. L. Wang, Z. Li, K. Cui, D. Karpuzov, X. H. Tan, A. Kohandehghan and D. Mitlin, Energy Environ. Sci., 2015, 8, 941-955.

12 X. Wang, S. Kajiyama, H. Iinuma, E. Hosono, S. Oro, I. Moriguchi, M. Okubo and A. Yamada, Nat. Commun., 2015, 6, 6544.

13 S. Komaba, T. Hasegawa, M. Dahbi and K. Kubota, Electrochem. Commun., 2015, 60, 172-175.

14 L. B. Dong, W. Yang, W. Yang, Y. Li, W. J. Wu and G. X. Wang, J. Mater. Chem. A, 2019, 7, 13810-13832.

15 G. Z. Fang, J. Zhou, A. Q. Pan and S. Q. Liang, ACS Energy Lett., 2018, 3, 2480-2501.

16 H. Jia, Z. Q. Wang, B. Tawiah, Y. D. Wang, C. Y. Chan, B. Fei and F. Pan, Nano Energy, 2020, 70, 104523.

17 Y. Li and H. Dai, Chem. Soc. Rev., 2014, 43, 5257-5275.

18 M. Song, H. Tan, D. L. Chao and H. J. Fan, Adv. Funct. Mater., 2018, 28, 1802564.

19 L. N. Chen, Q. Y. An and L. Q. Mai, Adv. Mater. Interfaces, 2019, 6, 1900387.

20 L. L. Zhang and X. S. Zhao, Chem. Soc. Rev., 2009, 38, 25202531. 
21 F. Wang, X. Wu, X. Yuan, Z. Liu, Y. Zhang, L. Fu, Y. Zhu, Q. Zhou, Y. Wu and W. Huang, Chem. Soc. Rev., 2017, 46, 6816-6854.

22 Y. Wang, Y. Song and Y. Xia, Chem. Soc. Rev., 2016, 45, 5925-5950.

23 L. Eliad, G. Salitra, A. Soffer and D. Aurbach, J. Phys. Chem. $B, 2001,105,6880-6887$.

24 J. Chmiola, G. Yushin, Y. Gogotsi, C. Portet, P. Simon and P. L. Taberna, Science, 2006, 313, 1760-1763.

25 M. Salanne, B. Rotenberg, K. Naoi, K. Kaneko, P. L. Taberna, C. P. Grey, B. Dunn and P. Simon, Nat. Energy, 2016, 1, 16070.

26 C. Prehal, C. Koczwara, N. Jackel, A. Schreiber, M. Burian, H. Amenitsch, M. A. Hartmann, V. Presser and O. Paris, Nat. Energy, 2017, 2, 16215.

27 J. Xu, N. Y. Yuan, J. M. Razal, Y. P. Zheng, X. S. Zhou, J. N. Ding, K. J. Cho, S. H. Ge, R. J. Zhang, Y. Gogotsi and R. H. Baughman, Energy Storage Mater., 2019, 22, 323-329.

28 C. Largeot, C. Portet, J. Chmiola, P. L. Taberna, Y. Gogotsi and P. Simon, J. Am. Chem. Soc., 2008, 130, 2730-2731.

29 D. T. Galhena, B. C. Bayer, S. Hofmann and G. A. Amaratunga, ACS Nano, 2016, 10, 747-754.

30 J. Chmiola, C. Largeot, P. L. Taberna, P. Simon and Y. Gogotsi, Angew. Chem., Int. Ed., 2008, 47, 3392-3395.

31 S. D. Han, N. N. Rajput, X. Qu, B. Pan, M. He, M. S. Ferrandon, C. Liao, K. A. Persson and A. K. Burrell, ACS Appl. Mater. Interfaces, 2016, 8, 3021-3031.

32 Y. Gogotsi and R. M. Penner, ACS Nano, 2018, 12, 20812083.

33 A. González, E. Goikolea, J. A. Barrena and R. Mysyk, Renewable Sustainable Energy Rev., 2016, 58, 1189-1206.

34 Q. Wang, J. Yan and Z. J. Fan, Energy Environ. Sci., 2016, 9, 729-762.

$35 \mathrm{H}$. Inoue, T. Morimoto and S. Nohara, Electrochem. SolidState Lett., 2007, 10, A261-A263.

36 A. Noori, M. F. El-Kady, M. S. Rahmanifar, R. B. Kaner and M. F. Mousavi, Chem. Soc. Rev., 2019, 48, 1272-1341.

37 L. B. Dong, X. P. Ma, Y. Li, L. Zhao, W. B. Liu, J. Y. Cheng, C. J. Xu, B. H. Li, Q. H. Yang and F. Y. Kang, Energy Storage Mater., 2018, 13, 96-102.

38 H. Zhang, Q. Liu, Y. Fang, C. Teng, X. Liu, P. Fang, Y. Tong and X. Lu, Adv. Mater., 2019, 31, e1904948.

39 Y. Y. Shao, Z. T. Sun, Z. N. Tian, S. Li, G. Q. Wu, M. L. Wang, X. L. Tong, F. Shen, Z. Xia, V. Tung, J. Y. Sun and Y. L. Shao, Adv. Funct. Mater., 2021, 31, 2007843.

40 X. Deng, J. Li, Z. Shan, J. Sha, L. Ma and N. Zhao, J. Mater. Chem. A, 2020, 8, 11617-11625.

41 W. J. Fan, J. Ding, J. N. Ding, Y. L. Zheng, W. Q. Song, J. F. Lin, C. X. Xiao, C. Zhong, H. L. Wang and W. B. Hu, Nano-Micro Lett., 2021, 13, 59.

42 H. Wang, W. Ye, Y. Yang, Y. Zhong and Y. Hu, Nano Energy, 2021, 85, 105942.

43 X. P. Ma, J. Y. Cheng, L. B. Dong, W. B. Liu, J. Mou, L. Zhao, J. J. Wang, D. Y. Ren, J. L. Wu, C. J. Xu and F. Y. Kang, Energy Storage Mater., 2019, 20, 335-342.

44 T. S. Mathis, N. Kurra, X. H. Wang, D. Pinto, P. Simon and Y. Gogotsi, Adv. Energy Mater., 2019, 9, 1902007.
45 A. Balducci, D. Belanger, T. Brousse, J. W. Long and W. Sugimoto, J. Electrochem. Soc., 2017, 164, A1487-A1488. 46 C. Choi, D. S. Ashby, D. M. Butts, R. H. DeBlock, Q. Wei, J. Lau and B. Dunn, Nat. Rev. Mater., 2019, 5, 5-19.

47 T. Brousse, D. Bélanger and J. W. Long, J. Electrochem. Soc., 2015, 162, A5185-A5189.

48 J. Yin, W. L. Zhang, N. A. Alhebshi, N. Salah and H. N. Alshareef, Small Methods, 2020, 4, 1900853.

49 Y. Tian, R. Amal and D.-W. Wang, Front. Energy Res., 2016, 4, 34 .

50 G. Q. Sun, H. S. Yang, G. F. Zhang, J. Gao, X. T. Jin, Y. Zhao, L. Jiang and L. T. Qu, Energy Environ. Sci., 2018, 11, 33673374 .

51 Y. C. Zhu, X. K. Ye, H. D. Jiang, J. X. Xia, Z. Y. Yue, L. H. Wang, Z. Q. Wan, C. Y. Jia and X. J. Yao, J. Power Sources, 2020, 453, 227851.

52 X. Zhang, Z. Pei, C. Wang, Z. Yuan, L. Wei, Y. Pan, A. Mahmood, Q. Shao and Y. Chen, Small, 2019, 15, e1903817.

53 Z. X. Pei, H. F. Li, Y. Huang, Q. Xue, Y. Huang, M. S. Zhu, Z. F. Wang and C. Y. Zhi, Energy Environ. Sci., 2017, 10, 742-749.

54 Z. J. Tang, Z. X. Pei, Z. F. Wang, H. F. Li, J. Zeng, Z. H. Ruan, Y. Huang, M. S. Zhu, Q. Xue, J. Yu and C. Y. Zhi, Carbon, 2018, 130, 532-543.

55 S. M. Chen, L. T. Ma, K. Zhang, M. Kamruzzaman, C. Y. Zhi and J. A. Zapien, J. Mater. Chem. A, 2019, 7, 7784-7790.

56 Z. Zhou, X. Zhou, M. Zhang, S. Mu, Q. Liu and Y. Tang, Small, 2020, 16, e2003174.

57 Z. W. Li, D. H. Chen, Y. F. An, C. L. Chen, L. Y. Wu, Z. J. Chen, Y. Sun and X. G. Zhang, Energy Storage Mater., 2020, 28, 307-314.

58 C. Wang, Z. Pei, Q. Meng, C. Zhang, X. Sui, Z. Yuan, S. Wang and Y. Chen, Angew. Chem., Int. Ed., 2021, 60, 990-997.

59 D. W. Wang, Z. M. Pan and Z. M. Lu, Microporous Mesoporous Mater., 2020, 306, 110445.

60 Z. X. Pei, Q. Q. Meng, L. Wei, J. Fan, Y. Chen and C. Y. Zhi, Energy Storage Mater., 2020, 28, 55-63.

61 P. G. Liu, Y. Gao, Y. Y. Tan, W. F. Liu, Y. P. Huang, J. Yan and K. Y. Liu, Nano Res., 2019, 12, 2835-2841.

62 Y. Y. Lu, Z. W. Li, Z. Y. Bai, H. Y. Mi, C. C. Ji, H. Pang, C. Yu and J. S. Qiu, Nano Energy, 2019, 66, 104132.

63 R. Yuksel, O. Buyukcakir, P. K. Panda, S. H. Lee, Y. Jiang, D. Singh, S. Hansen, R. Adelung, Y. K. Mishra, R. Ahuja and R. S. Ruoff, Adv. Funct. Mater., 2020, 30, 1909725.

64 Y. G. Lee and G. H. An, ACS Appl. Mater. Interfaces, 2020, 12, 41342-41349.

65 D. W. Wang, S. Y. Wang and Z. M. Lu, Int. J. Energy Res., 2021, 45, 2498-2510.

66 Z. Pei, J. Gu, Y. Wang, Z. Tang, Z. Liu, Y. Huang, Y. Huang, J. Zhao, Z. Chen and C. Zhi, ACS Nano, 2017, 11, 6004-6014.

67 S. Wang, Q. Wang, W. Zeng, M. Wang, L. Ruan and Y. Ma, Nano-Micro Lett., 2019, 11, 70.

68 Q. Chen, J. Jin, Z. Kou, C. Liao, Z. Liu, L. Zhou, J. Wang and L. Mai, Small, 2020, 16, e2000091. 
69 L. B. Dong, W. Yang, W. Yang, C. Y. Wang, Y. Li, C. J. Xu, S. W. Wan, F. R. He, F. Y. Kang and G. X. Wang, NanoMicro Lett., 2019, 11, 94.

70 X. Ma, J. Wang, X. Wang, L. Zhao and C. Xu, J. Mater. Sci.: Mater. Electron., 2019, 30, 5478-5486.

71 T. C. An and W. L. Cheng, J. Mater. Chem. A, 2018, 6, 1547815494.

72 J. Han, K. Wang, W. Liu, C. Li, X. Sun, X. Zhang, Y. An, S. Yi and Y. Ma, Nanoscale, 2018, 10, 13083-13091.

73 N. Wang, T. Xin, Y. Zhao, Q. Li, M. J. Hu and J. Z. Liu, ACS Sustainable Chem. Eng., 2019, 7, 14195-14202.

74 Y. Huang, H. F. Li, Z. F. Wang, M. S. Zhu, Z. X. Pei, Q. Xue, Y. Huang and C. Y. Zhi, Nano Energy, 2016, 22, 422-438.

75 Y. Zhao, Y. N. Wang, Z. M. Zhao, J. W. Zhao, T. Xin, N. Wang and J. Z. Liu, Energy Storage Mater., 2020, 28, 64-72.

76 J. Pang, R. G. Mendes, A. Bachmatiuk, L. Zhao, H. Q. Ta, T. Gemming, H. Liu, Z. Liu and M. H. Rummeli, Chem. Soc. Rev., 2019, 48, 72-133.

77 M. S. Zhu, Y. Huang, Q. H. Deng, J. Zhou, Z. X. Pei, Q. Xue, Y. Huang, Z. F. Wang, H. F. Li, Q. Huang and C. Y. Zhi, Adv. Energy Mater., 2016, 6, 1600969.

78 Q. Wang, S. L. Wang, X. H. Guo, L. M. Ruan, N. Wei, Y. Ma, J. Y. Li, M. Wang, W. Q. Li and W. Zeng, Adv. Electron. Mater., 2019, 5, 1900537.

79 X. L. Li, M. Li, Q. Yang, D. H. Wang, L. T. Ma, G. J. Liang, Z. D. Huang, B. B. Dong, Q. Huang and C. Y. Zhi, Adv. Energy Mater., 2020, 10, 2001394.

80 Z. D. Huang, A. Chen, F. N. Mo, G. J. Liang, X. L. Li, Q. Yang, Y. Guo, Z. Chen, Q. Li, B. B. Dong and C. Y. Zhi, Adv. Energy Mater., 2020, 10, 2001024.

81 G. H. An, Appl. Surf. Sci., 2020, 530, 147220.

82 T. Xiong, Y. Shen, W. S. V. Lee and J. Xue, Nano Mater. Sci., 2020, 2, 159-163.

83 P. F. Yu, Y. Zeng, Y. X. Zeng, H. W. Dong, H. Hu, Y. L. Liu, M. T. Zheng, Y. Xiao, X. H. Lu and Y. R. Liang, Electrochim. Acta, 2019, 327, 134999.

84 S. L. Wu, Y. T. Chen, T. P. Jiao, J. Zhou, J. Y. Cheng, B. Liu, S. R. Yang, K. L. Zhang and W. J. Zhang, Adv. Energy Mater., 2019, 9, 1902915.

85 H. Wang, M. Wang and Y. B. Tang, Energy Storage Mater., 2018, 13, 1-7.

86 H. T. Zhou, C. Liu, J. C. Wu, M. H. Liu, D. Zhang, H. L. Song, X. Y. Zhang, H. Q. Gao, J. H. Yang and D. Chen, J. Mater. Chem. A, 2019, 7, 9708-9715.

87 L. Han, H. L. Huang, J. F. Li, X. L. Zhang, Z. L. Yang, M. Xu and L. K. Pan, J. Mater. Chem. A, 2020, 8, 15042-15050.

88 M. F. Chen, J. Z. Chen, W. J. Zhou, J. L. Xu and C. P. Wong, J. Mater. Chem. A, 2019, 7, 26524-26532.

89 P. Zhang, Y. Li, G. Wang, F. Wang, S. Yang, F. Zhu, X. Zhuang, O. G. Schmidt and X. Feng, Adv. Mater., 2019, 31, e1806005.

90 H. Ma, H. Chen, M. Wu, F. Chi, F. Liu, J. Bai, H. Cheng, C. Li and L. Qu, Angew. Chem., Int. Ed., 2020, 59, 14541-14549.

91 Z. Huang, T. Wang, H. Song, X. Li, G. Liang, D. Wang, Q. Yang, Z. Chen, L. Ma, Z. Liu, B. Gao, J. Fan and C. Zhi, Angew. Chem., Int. Ed., 2021, 60, 1011-1021.
92 P. A. Maughan, N. Tapia-Ruiz and N. Bimbo, Electrochim. Acta, 2020, 341, 136061.

93 Z. Fan, J. Jin, C. Li, J. Cai, C. Wei, Y. Shao, G. Zou and J. Sun, ACS Nano, 2021, 15, 3098-3107.

94 J. Shi, S. Wang, Q. Wang, X. Chen, X. Du, M. Wang, Y. Zhao, C. Dong, L. Ruan and W. Zeng, J. Power Sources, 2020, 446, 227345.

95 F. Z. Cui, Z. C. Liu, D. L. Ma, L. L. Liu, T. Huang, P. P. Zhang, D. M. Tan, F. X. Wang, G. F. Jiang and Y. P. Wu, Chem. Eng. J., 2021, 405, 127038.

96 N. Zhang, F. Cheng, Y. Liu, Q. Zhao, K. Lei, C. Chen, X. Liu and J. Chen, J. Am. Chem. Soc., 2016, 138, 12894-12901.

97 M. Hartmann, T. Clark and R. vanEldik, J. Am. Chem. Soc., 1997, 119, 7843-7850.

98 S. De, S. M. Ali, A. Ali and V. G. Gaikar, Phys. Chem. Chem. Phys., 2009, 11, 8285-8294.

99 C. Y. Chen, K. Matsumoto, K. Kubota, R. Hagiwara and Q. Xu, Adv. Energy Mater., 2019, 9, 1900196.

100 Z. Liu, G. Li, T. Cui, A. Borodin, C. Kuhl and F. Endres, J. Solid State Electrochem., 2017, 22, 91-101.

101 L. Suo, O. Borodin, T. Gao, M. Olguin, J. Ho, X. Fan, C. Luo, C. Wang and K. Xu, Science, 2015, 350, 938-943.

102 F. Wang, O. Borodin, T. Gao, X. Fan, W. Sun, F. Han, A. Faraone, J. A. Dura, K. Xu and C. Wang, Nat. Mater., 2018, 17, 543-549.

103 C. Zhang, J. Holoubek, X. Wu, A. Daniyar, L. Zhu, C. Chen, D. P. Leonard, I. A. Rodriguez-Perez, J. X. Jiang, C. Fang and X. Ji, Chem. Commun., 2018, 54, 14097-14099.

104 J. Xie, Z. Liang and Y. C. Lu, Nat. Mater., 2020, 19, 10061011.

105 Y. J. Zhang, Z. Chen, H. Y. Qiu, W. H. Yang, Z. M. Zhao, J. W. Zhao and G. L. Cui, NPG Asia Mater., 2020, 12, 4.

106 E. Staunton, A. M. Christie, I. Martin-Litas, Y. G. Andreev, A. M. Slawin and P. G. Bruce, Angew. Chem., Int. Ed., 2004, 43, 2103-2105.

107 Y. Xu, Y. Zhao, J. Ren, Y. Zhang and H. Peng, Angew. Chem., Int. Ed., 2016, 55, 7979-7982.

108 T. Ni, S. L. Wang, J. J. Shi, X. Y. Du, Q. H. Cheng, Z. Y. Dong, L. M. Ruan, W. Zeng, X. H. Guo, X. G. Ren and Z. X. Huang, Adv. Mater. Technol., 2020, 5, 2000268.

109 Z. Jian, N. J. Yang, M. Vogel, S. Leith, A. Schulte, H. Schonherr, T. P. Jiao, W. J. Zhang, J. Muller, B. Butz and X. Jiang, Adv. Energy Mater., 2020, 10, 2002202.

110 Y. W. Zheng, W. Zhao, D. D. Jia, Y. Liu, L. Cui, D. Wei, R. K. Zheng and J. Q. Liu, Chem. Eng. J., 2020, 387, 124161.

111 D. Oran, S. G. Rodriques, R. Gao, S. Asano, M. A. SkylarScott, F. Chen, P. W. Tillberg, A. H. Marblestone and E. S. Boyden, Science, 2018, 362, 1281-1285.

112 Z. Pei, Z. Yuan, C. Wang, S. Zhao, J. Fei, L. Wei, J. Chen, C. Wang, R. Qi, Z. Liu and Y. Chen, Angew. Chem., Int. Ed., 2020, 59, 4793-4799.

113 K. Wang, X. Zhang, J. Han, X. Zhang, X. Sun, C. Li, W. Liu, Q. Li and Y. Ma, ACS Appl. Mater. Interfaces, 2018, 10, 24573-24582.

114 S. Wu, M. Hua, Y. Alsaid, Y. Du, Y. Ma, Y. Zhao, C. Y. Lo, C. Wang, D. Wu, B. Yao, J. Strzalka, H. Zhou, X. Zhu and X. He, Adv. Mater., 2021, 33, e2007829. 
115 M. Xu, D. G. Ivey, Z. Xie and W. Qu, J. Power Sources, 2015, 283, 358-371.

116 S. B. Aziz, T. J. Woo, M. F. Z. Kadir and H. M. Ahmed, J. Sci.: Adv. Mater. Devices, 2018, 3, 1-17.

117 G. M. Wu, S. J. Lin and C. C. Yang, J. Membr. Sci., 2006, 275, 127-133.

118 L. Han, H. L. Huang, X. B. Fu, J. F. Li, Z. L. Yang, X. J. Liu, L. K. Pan and M. Xu, Chem. Eng. J., 2020, 392, 123733.

119 X. B. Cheng, T. Z. Hou, R. Zhang, H. J. Peng, C. Z. Zhao, J. Q. Huang and Q. Zhang, Adv. Mater., 2016, 28, 2888-2895.

120 W. Lu, C. Xie, H. Zhang and X. Li, ChemSusChem, 2018, 11, 3996-4006.

121 C. Han, W. J. Li, H. K. Liu, S. X. Dou and J. Z. Wang, Nano Energy, 2020, 74, 104880.

122 Z. M. Zhao, J. W. Zhao, Z. L. Hu, J. D. Li, J. J. Li, Y. J. Zhang, C. Wang and G. L. Cui, Energy Environ. Sci., 2019, 12, 19381949.

123 Q. Li, Y. Zhao, F. Mo, D. Wang, Q. Yang, Z. Huang, G. Liang, A. Chen and C. Zhi, EcoMat, 2020, 2, e12035.

124 Q. Yang, G. Liang, Y. Guo, Z. Liu, B. Yan, D. Wang, Z. Huang, X. Li, J. Fan and C. Zhi, Adv. Mater., 2019, 31, e1903778.

125 J. W. Zhao, J. Zhang, W. H. Yang, B. B. Chen, Z. M. Zhao, H. Y. Qiu, S. M. Dong, X. H. Zhou, G. L. Cui and L. Q. Chen, Nano Energy, 2019, 57, 625-634.

126 Z. Wang, J. H. Huang, Z. W. Guo, X. L. Dong, Y. Liu, Y. G. Wang and Y. Y. Xia, Joule, 2019, 3, 1289-1300.

127 Q. Zhang, J. Luan, Y. Tang, X. Ji and H. Wang, Angew. Chem., Int. Ed., 2020, 59, 13180-13191.

128 Q. Zhang, J. Y. Luan, Y. G. Tang, X. B. Ji, S. Y. Wang and H. Y. Wang, J. Mater. Chem. A, 2018, 6, 18444-18448.

129 X. S. Xie, S. Q. Liang, J. W. Gao, S. Guo, J. B. Guo, C. Wang, G. Y. Xu, X. W. Wu, G. Chen and J. Zhou, Energy Environ. Sci., 2020, 13, 503-510.

130 Y. Zeng, X. Zhang, R. Qin, X. Liu, P. Fang, D. Zheng, Y. Tong and X. Lu, Adv. Mater., 2019, 31, 1903675.

131 J. Zheng, Q. Zhao, T. Tang, J. Yin, C. D. Quilty, G. D. Renderos, X. Liu, Y. Deng, L. Wang, D. C. Bock, C. Jaye, D. Zhang, E. S. Takeuchi, K. J. Takeuchi, A. C. Marschilok and L. A. Archer, Science, 2019, 366, 645648.

132 Q. Zhang, J. Luan, L. Fu, S. Wu, Y. Tang, X. Ji and H. Wang, Angew. Chem., Int. Ed., 2019, 58, 15841-15847.

133 W. N. Xu, K. N. Zhao, W. C. Huo, Y. Z. Wang, G. Yao, X. Gu, H. W. Cheng, L. Q. Mai, C. G. Hu and X. D. Wang, Nano Energy, 2019, 62, 275-281.

134 H. F. Li, C. P. Han, Y. Huang, Y. Huang, M. S. Zhu, Z. X. Pei, Q. Xue, Z. F. Wang, Z. X. Liu, Z. J. Tang, Y. K. Wang, F. Y. Kang, B. H. Li and C. Y. Zhi, Energy Environ. Sci., 2018, 11, 941-951.

135 D. Chao, C. R. Zhu, M. Song, P. Liang, X. Zhang, N. H. Tiep, H. Zhao, J. Wang, R. Wang, H. Zhang and H. J. Fan, Adv. Mater., 2018, 30, e1803181.

136 Y. Tian, Y. An, C. Wei, B. Xi, S. Xiong, J. Feng and Y. Qian, ACS Nano, 2019, 13, 11676-11685.
137 S. Y. Su, Y. Xu, Y. Wang, X. Y. Wang, L. Shi, D. Wu, P. C. Zou, A. Nairan, Z. Y. Lin, F. Y. Kang and C. Yang, Chem. Eng. J., 2019, 370, 330-336.

138 C. P. Li, X. D. Shi, S. Q. Liang, X. M. Ma, M. M. Han, X. W. Wu and J. Zhou, Chem. Eng. J., 2020, 379, 122248.

139 Y. Yin, S. Wang, Q. Zhang, Y. Song, N. Chang, Y. Pan, H. Zhang and X. Li, Adv. Mater., 2020, 32, e1906803.

140 Z. J. Zhang, D. B. Zhou, X. J. Bao, L. Zhou, J. Zhao and B. Y. Huang, J. Solid State Electrochem., 2018, 22, 3775-3783.

141 Z. G. Li, W. J. Deng, C. Li, W. J. Wang, Z. Q. Zhou, Y. B. Li, X. R. Yuan, J. Hu, M. Zhang, J. L. Zhu, W. Tang, X. Wang and R. Li, J. Mater. Chem. A, 2020, 8, 17725-17731.

142 M. Zhu, J. Hu, Q. Lu, H. Dong, D. D. Karnaushenko, C. Becker, D. Karnaushenko, Y. Li, H. Tang, Z. Qu, J. Ge and O. G. Schmidt, Adv. Mater., 2021, 33, e2007497.

143 L. T. Kang, M. W. Cui, F. Y. Jiang, Y. F. Gao, H. J. Luo, J. J. Liu, W. Liang and C. Y. Zhi, Adv. Energy Mater., 2018, 8, 1801090.

144 P. C. Liang, J. Yi, X. Y. Liu, K. Wu, Z. Wang, J. Cui, Y. Y. Liu, Y. G. Wang, Y. Y. Xia and J. J. Zhang, Adv. Funct. Mater., 2020, 30, 1908528.

145 D. Han, S. Wu, S. Zhang, Y. Deng, C. Cui, L. Zhang, Y. Long, H. Li, Y. Tao, Z. Weng, Q. H. Yang and F. Kang, Small, 2020, 16, e2001736.

146 M. Liu, L. Yang, H. Liu, A. Amine, Q. Zhao, Y. Song, J. Yang, K. Wang and F. Pan, ACS Appl. Mater. Interfaces, 2019, 11, 32046-32051.

147 P. G. Liu, W. F. Liu, Y. P. Huang, P. L. Li, J. Yan and K. Y. Liu, Energy Storage Mater., 2020, 25, 858-865.

148 L. B. Dong, W. Yang, W. Yang, H. Tian, Y. F. Huang, X. L. Wang, C. J. Xu, C. Y. Wang, F. Y. Kang and G. X. Wang, Chem. Eng. J., 2020, 384, 123355.

149 R. Yuksel, O. Buyukcakir, W. K. Seong and R. S. Ruoff, Adv. Energy Mater., 2020, 10, 1904215.

150 J. Hao, B. Li, X. Li, X. Zeng, S. Zhang, F. Yang, S. Liu, D. Li, C. Wu and Z. Guo, Adv. Mater., 2020, 32, e2003021.

151 Y. Yang, C. Liu, Z. Lv, H. Yang, Y. Zhang, M. Ye, L. Chen, J. Zhao and C. C. Li, Adv. Mater., 2021, 33, e2007388.

152 Y. Tian, Y. L. An, C. L. Wei, B. J. Xi, S. L. Xiong, J. K. Feng and Y. T. Qian, Adv. Energy Mater., 2021, 11, 2002529.

153 F. Wan, L. Zhang, X. Dai, X. Wang, Z. Niu and J. Chen, Nat. Commun., 2018, 9, 1656.

154 R. Z. Qin, Y. T. Wang, M. Z. Zhang, Y. Wang, S. X. Ding, A. Y. Song, H. C. Yi, L. Y. Yang, Y. L. Song, Y. H. Cui, J. Liu, Z. Q. Wang, S. N. Li, Q. H. Zhao and F. Pan, Nano Energy, 2021, 80, 105478.

155 S. J. Banik and R. Akolkar, Electrochim. Acta, 2015, 179, 475481.

156 Y. Huang, Z. Li, Z. X. Pei, Z. X. Liu, H. F. Li, M. S. Zhu, J. Fan, Q. B. Dai, M. D. Zhang, L. M. Dai and C. Y. Zhi, Adv. Energy Mater., 2018, 8, 1802288.

157 C. Lin, S. S. Shinde, X. Li, D. H. Kim, N. Li, Y. Sun, X. Song, H. Zhang, C. H. Lee, S. U. Lee and J. H. Lee, ChemSusChem, 2018, 11, 3215-3224.

158 T. C. Li, D. Fang, J. Zhang, M. E. Pam, Z. Y. Leong, J. Yu, X. L. Li, D. Yan and H. Y. Yang, J. Mater. Chem. A, 2021, 9, 6013-6028. 
159 H. Li, Z. Tang, Z. Liu and C. Zhi, Joule, 2019, 3, 613-619. 160 N. Pandharinath, Aviation Meteorology, BS Publications, 2014.

161 L. Y. Yang, L. Song, Y. Feng, M. J. Cao, P. C. Zhang, X. F. Zhang and J. F. Yao, J. Mater. Chem. A, 2020, 8, 12314-12318.

162 Q. Zhang, Y. Ma, Y. Lu, L. Li, F. Wan, K. Zhang and J. Chen, Nat. Commun., 2020, 11, 4463.

163 P. H. Yang, C. Z. Feng, Y. P. Liu, T. Cheng, X. L. Yang, H. D. Liu, K. Liu and H. J. Fan, Adv. Energy Mater., 2020, 10, 2002898.

164 K. Ge and G. Liu, Chem. Commun., 2019, 55, 7167-7170.

165 L. O. O. Ma, Y. W. Zhao, X. X. Ji, J. Zeng, Q. Yang, Y. Guo, Z. D. Huang, X. L. Li, J. Yu and C. Y. Zhi, Adv. Energy Mater., 2019, 9, 1900509.

166 B. D. Boruah, A. Mathieson, B. Wen, C. Jo, F. Deschler and M. De Volder, Nano Lett., 2020, 20, 5967-5974.
167 B. D. Boruah, B. Wen, S. Nagane, X. Zhang, S. D. Stranks, A. Boies and M. De Volder, ACS Energy Lett., 2020, 5, 3132-3139.

168 G. Sun, Y. Xiao, B. Lu, X. Jin, H. Yang, C. Dai, X. Zhang, Y. Zhao and L. Qu, ACS Appl. Mater. Interfaces, 2020, 12, 7239-7248.

169 J. Zeng, L. B. Dong, L. L. Sun, W. Wang, Y. H. Zhou, L. Wei and X. Guo, Nano-Micro Lett., 2020, 13, 19.

170 S. Jiao, A. Zhou, M. Wu and H. Hu, Adv. Sci., 2019, 6, 1900529.

171 Y. Wu, H. Hu, C. Yuan, J. Song and M. Wu, Nano Energy, 2020, 74, 104812.

172 Z. Li, J. Song, H. Hu, C. Yuan, M. Wu and D. Ho, J. Mater. Chem. A, 2021, 9, 2899-2911.

173 Y. Gogotsi and P. Simon, Science, 2011, 334, 917-918. 